University of Tennessee Health Science Center

UTHSC Digital Commons

\title{
Spatial Resolution Analysis of a Variable Resolution X-ray Cone- beam Computed Tomography System
}

\author{
Bahram Dahi \\ University of Tennessee Health Science Center
}

Follow this and additional works at: https://dc.uthsc.edu/dissertations

Part of the Analytical, Diagnostic and Therapeutic Techniques and Equipment Commons, and the Medical Sciences Commons

\section{Recommended Citation}

Dahi, Bahram, "Spatial Resolution Analysis of a Variable Resolution X-ray Cone-beam Computed Tomography System" (2009). Theses and Dissertations (ETD). Paper 59. http://dx.doi.org/10.21007/ etd.cghs.2009.0063.

This Dissertation is brought to you for free and open access by the College of Graduate Health Sciences at UTHSC Digital Commons. It has been accepted for inclusion in Theses and Dissertations (ETD) by an authorized administrator of UTHSC Digital Commons. For more information, please contact jwelch30@uthsc.edu. 


\title{
Spatial Resolution Analysis of a Variable Resolution X-ray Cone-beam Computed Tomography System
}

\begin{abstract}
A new cone-beam computed tomography (CBCT) system is designed and implemented that can adaptively provide high resolution CT images for objects of different sizes. The new system, called Variable Resolution X-ray Cone-beam CT (VRX-CBCT) uses a Csl-based amorphous silicon flat panel detector (FPD) that can tilt about its horizontal $(u)$ axis and vertical $(v)$ axis independently. The detector angulation improves the spatial resolution of the CT images by changing the effective size of each detector cell. Two components of spatial resolution of the system, namely the transverse and axial modulation transfer functions (MTF), are analyzed in three different situations: (1) when the FPD is tilted only about its vertical axis $(v),(2)$ when the FPD is tilted only about its horizontal axis $(u)$, and (3) when the FPD is tilted isotropically about both its vertical and horizontal axes. Custom calibration and MTF phantoms were designed and used to calibrate and measure the spatial resolution of the system for each case described above. A new 3D reconstruction algorithm was developed and tested for the VRX-CBCT system, which combined with a novel 3D reconstruction algorithm, has improved the overall resolution of the system compared to an FDK-based algorithm.
\end{abstract}

\section{Document Type}

Dissertation

Degree Name

Doctor of Philosophy $(\mathrm{PhD})$

\section{Program}

Biomedical Engineering and Imaging

\section{Research Advisor}

Gary S. Keyes, Ph.D.

\section{Keywords}

CBCT, CT Reconstruction, FPD, MTF, Spatial Resolution, VRX-CBCT

\section{Subject Categories}

Analytical, Diagnostic and Therapeutic Techniques and Equipment | Medical Sciences | Medicine and Health Sciences 


\title{
SPATIAL RESOLUTION ANALYSIS OF A VARIABLE RESOLUTION X-RAY CONE-BEAM COMPUTED TOMOGRAPHY SYSTEM
}

\author{
A Dissertation \\ Presented for \\ The Graduate Studies Council \\ The University of Tennessee \\ Health Science Center
}

\begin{abstract}
In Partial Fulfillment
Of the Requirements for the Degree

Doctor of Philosophy

In the Joint Graduate Program in Biomedical Engineering and Imaging

From The University of Tennessee

and

The University of Memphis
\end{abstract}

By

Bahram Dahi

May 2009 
Portions of chapters 3-5 (C) 2009 by SPIE.

All other material (C) 2009 by Bahram Dahi. All rights reserved. 
To my father, Reza Dahi, who never stopped guiding me.

To my mother, Koukab Vaziri, who never stopped supporting me.

And to my wife, Christy Wilson, who never stopped believing in me.

I am a better person today because of you. 


\section{Acknowledgements}

I would like to thank Dr. Gary Keyes, for being an effective research advisor, a caring mentor, and a supporting friend throughout my years at the University of Tennessee Health Science Center. His vast knowledge in science and engineering along with his invaluable experience in management, guided me through the ups and downs of my research days. My sincere gratitude to Dr. Frank DiBianca, who introduced me to this area of research, and provided me with tremendous help and support. I am here today because they never stopped believing in me.

I would also like to thank all other members of my graduate committee: Dr. Waleed Gaber, who has been a supporter and a true friend, Dr. Richard Smith, provided me with much needed medical and practical perspective, and Dr. Herbert Zeman, whose knowledge in the field of BioImaging effectively guided in my research. I would like to thank Joseph Laughter, Dr. Roman Melnyk, and Sowseelya Arikapudi for all their help in hardware construction, design improvement, and experiments. Many thanks to my friend and colleague, David Rendon, for his overwhelming support throughout my research and especially when it came to improving the algorithms. I thank the University of Tennesse Health Science Center for providing all the resources for me to be successful. And last but not least, I want to thank my lovely wife and my parents for supporting me throughout my life and my research career.

This study was partially supported by the NIH Grant No. EB-00418. 


\section{Abstract}

A new cone-beam computed tomography (CBCT) system is designed and implemented that can adaptively provide high resolution CT images for objects of different sizes. The new system, called Variable Resolution X-ray Cone-beam CT (VRXCBCT) uses a CsI-based amorphous silicon flat panel detector (FPD) that can tilt about its horizontal $(u)$ axis and vertical $(v)$ axis independently. The detector angulation improves the spatial resolution of the $\mathrm{CT}$ images by changing the effective size of each detector cell. Two components of spatial resolution of the system, namely the transverse and axial modulation transfer functions (MTF), are analyzed in three different situations: (1) when the FPD is tilted only about its vertical axis $(v),(2)$ when the FPD is tilted only about its horizontal axis $(u)$, and (3) when the FPD is tilted isotropically about both its vertical and horizontal axes. Custom calibration and MTF phantoms were designed and used to calibrate and measure the spatial resolution of the system for each case described above. A new 3D reconstruction algorithm was developed and tested for the VRX-CBCT system, which combined

with a novel 3D reconstruction algorithm, has improved the overall resolution of the system compared to an FDK-based algorithm. 


\section{Table of Contents}

$\begin{array}{lll}\text { Chapter } 1 & \text { Introduction } & 1\end{array}$

Chapter 2 Background 3

2.1 Characteristics of X-rays in Diagnostic Imaging . . . . . . . . . 3

2.2 How X-rays Are Detected $\ldots \ldots \ldots \ldots$

2.2.1 Film Screen Detector System _ . . . . . . . . . . . 6

2.2 .2 Image Intensifiers . . . . . . . . . . . . . . . . . 8

2.2 .3 Digital Detectors . . . . . . . . . . . . . 8

2.2.3.1 Linear Detector Arrays _... . . . . . . 8

2.2.3.2 Flat Panel Detectors . . . . . . . . . . . . . . 9

2.3 Current Uses of X-rays in Diagnostic Imaging $\ldots \ldots \ldots \ldots$

2.3.1 Projection Radiography _. . . . . . . . . . . . . . 10

2.3 .2 Mammography . . . . . . . . . . . . . . . . . . . 10

2.3 .3 Fluoroscopy . . . . . . . . . . . . . . . . . . . . 10

2.3 .4 Tomosynthesis . . . . . . . . . . . . . . . . . 12

2.4 Computed Tomography Imaging . . . . . . . . . . . . . . . . 13

2.4.1 Conventional CT . . . . . . . . . . . . . . . . . 13

2.4 .2 Cone-beam CT . . . . . . . . . . . . . . . . 15

2.4 .3 Micro-CT Imaging . . . . . . . . . . . . . . . . . . . 16

2.5 Variable Resolution X-ray CT System . . . . . . . . . . . . . 17

2.6 Modulation Transfer Function . . . . . . . . . . . . . . . 20

Chapter 3 Variable Resolution X-ray Cone-beam CT (VRX-CBCT) 22

3.1 Introduction . . . . . . . . . . . . . . . . . 22

3.2 Design and Methods . . . . . . . . . . . . . . . 23

3.2 .1 Basic Components . . . . . . . . . . . . . . . . 23

3.2.1.1 Flat Panel Detector . . . . . . . . . . . . . . . 23

3.2.1.2 Micro-focal X-ray Tube . . . . . . . . . . . . 23 
3.2.1.3 Rotary Table . . . . . . . . . . . . . . . . . 24

3.2.1.4 Collimation . . . . . . . . . . . . . . . . 24

3.2 .2 Terminology . . . . . . . . . . . . . . . . . . . . 24

3.3 Transverse Spatial Resolution Analysis _. . . . . . . . . . . . . 28

3.3.1 Experiment Setup . . . . . . . . . . . . . . . . . . . . . 28

3.3.1.1 Flat Panel Detector . . . . . . . . . . . . . . 28

3.3.1.2 MTF Phantom . . . . . . . . . . . . . . . 30

3.3.1.3 System Parameters . . . . . . . . . . . . . . . 30

3.3.2 FPD Pre-processing and Data Acquisition . . . . . . . . 30

3.3 .3 Calibration . . . . . . . . . . . . . . . . 30

3.3.4 CT Reconstruction and MTF Calculation . . . . . . . . 35

3.3.4.1 CT Reconstruction Algorithm . . . . . . . . . . . 35

3.3.4.2 MTF Calculations . . . . . . . . . . . . 36

3.4 Axial Spatial Resolution Analysis . . . . . . . . . . . . . . . . 36

3.4 .1 Experiment Setup . . . . . . . . . . . . . . . . . . . . 38

3.4.1.1 Flat Panel Detector . . . . . . . . . . . . . . 38

3.4.1.2 MTF Phantom . . . . . . . . . . . . . . 38

3.4.1.3 System Parameters . . . . . . . . . . . . . . . . 40

3.4.2 FPD Pre-processing and Data Acquisition . . . . . . . . . 40

3.4 .3 Calibration . . . . . . . . . . . . . . . . 41

3.4.4 CT Reconstruction and MTF Calculation . . . . . . . . . 42

3.4.4.1 CT Reconstruction Algorithm . . . . . . . . . . . . 42

3.4.4.2 MTF Calculations . . . . . . . . . . . . . 42

3.5 Isotropic Spatial Resolution Analysis . . . . . . . . . . . . . . . 43

3.5.1 Experiment Setup . . . . . . . . . . . . . . . . . . . 43

3.5.1.1 Flat Panel Detector . . . . . . . . . . . . . . 43

3.5.1.2 MTF Phantom . . . . . . . . . . . . . . . . 44

3.5.1.3 System Parameters . . . . . . . . . . . . . . . . 46

3.5.2 FPD Pre-processing and Data Acquisition . . . . . . . . . 46

3.5 .3 Calibration . . . . . . . . . . . . . . 47

3.5.3.1 Basic Concept . . . . . . . . . . . . . 47 
3.5.3.2 Implementation . . . . . . . . . . . . . . . . . . 49

3.5.4 CT Reconstruction and MTF Calculation . . . . . . . . 52

3.5.4.1 CT Reconstruction Algorithm . . . . . . . . . . 52

3.5.4.2 MTF Calculations . . . . . . . . . . . . 54

Chapter 4 Results and Discussion $\quad 56$

4.1 Transverse Spatial Resolution of the First Generation VRX-CBCT . . 56

4.2 Axial Spatial Resolution of the Second Generation VRX-CBCT . . 58

4.3 Comprehensive Spatial Resolution of the Isotropic VRX-CBCT _ . . 63

$\begin{array}{lll}\text { Chapter } 5 & \text { Conclusions } & 74\end{array}$

5.1 Limitations . . . . . . . . . . . . . . . . . . . . . 74

5.2 Potential Applications . . . . . . . . . . . . . 76

$\begin{array}{ll}\text { List of References } & 77\end{array}$

$\begin{array}{lll}\text { Appendix A } & \text { VRX-CBCT Calibration Code } & 83\end{array}$

$\begin{array}{lll}\text { Appendix B VRX-CBCT Reconstruction Code } & 93\end{array}$

$\begin{array}{ll}\text { Vita } & 102\end{array}$ 


\section{List of Tables}

3.1 Flat panel detector specifications . . . . . . . . . . . . . . . 24

3.2 X-ray tube specifications . . . . . . . . . . . . . . . . . 24

3.3 VRX-CBCT parameters for transverse resolution analysis . . . . . . . . 31

3.4 VRX-CBCT parameters for axial resolution analysis . . . . . . . . . 41

3.5 VRX-CBCT parameters for isotropic resolution analysis . . . . . . . . 46

4.1 Selected frequencies and MTF values of the VRX-CBCT system . . . . . 60 


\section{List of Figures}

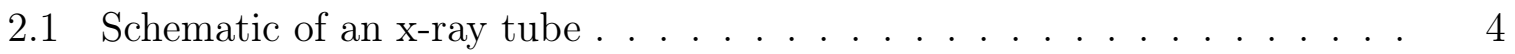

2.2 Heel effect in x-ray tubes . . . . . . . . . . . . . . . . . . 7

2.3 Comparison of linear array and flat panel detectors . . . . . . . . . 11

2.4 Generations of clinical CT scanners . . . . . . . . . . . . . . . . 14

2.5 X-ray detector configurations $\ldots \ldots \ldots \ldots \ldots$

2.6 Generational configurations of the VRX-CT system . . . . . . . . . 19

3.1 Adjustable acrylic stand for the rotary table . . . . . . . . . . 25

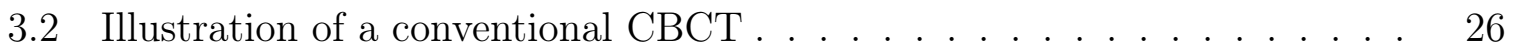

3.3 First generation VRX-CBCT test bed . . . . . . . . . . . . . 29

3.4 Transverse MTF phantom . . . . . . . . . . . . . . . . . . . . . 31

3.5 Calibration phantom for the first generation VRX-CBCT . . . . . . . 33

3.6 Example of a pin sinogram at the midplane . . . . . . . . . . . . 34

3.7 Second generation of the VRX-CBCT test bed . . . . . . . . . . 37

3.8 FPD rotation system in the second generation VRX-CBCT . . . . . . . 39

3.9 VRX-CBCT alignment . . . . . . . . . . . . . . . . . . . . . . . 39

3.10 Axial MTF phantom . . . . . . . . . . . . . . . . . 40

3.11 The isotropic MTF phantom . . . . . . . . . . . . . . . 45

3.12 Geometry of the isotropic VRX-CBCT . . . . . . . . . . . 48

3.13 3D calibration phantom . . . . . . . . . . . . . . 50

3.14 NSSE three-stage minimization $\ldots \ldots \ldots \ldots \ldots \ldots$

4.1 VRX-CBCT images taken at different vr $_{v}$ angles . . . . . . . . . 57

4.2 Transverse system MTF curves of the VRX-CBCT . . . . . . . . . . 59

4.3 A VRX-CBCT image of a piece of beta-tricalcium phosphate scaffold . . 61

4.4 Sagittal views of the axial MTF phantom . . . . . . . . . . . . . . . 62

4.5 Axial spatial resolution of the VRX-CBCT system at various vr $x_{u}$

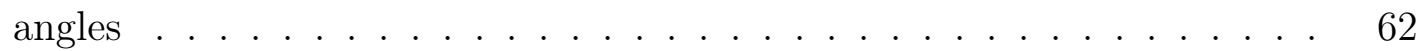


4.6 Reconstructed CT images of a slightly stretched coiled spring . . . . . . 64

4.7 VRX-CBCT images of an isotropic MTF phantom . . . . . . . . . . . . 65

4.8 Transverse spatial resolution of the VRX-CBCT system at different vr $x_{v}$ angles using the 3D reconstruction algorithm . . . . . . . . . . . 66

4.9 Axial spatial resolution of the VRX-CBCT system at different vr $x_{v}$ angles using the 3D reconstruction algorithm . . . . . . . . . . 67

4.10 Axial spatial resolution of the VRX-CBCT system at different vr $x_{u}$ angles using the 3D reconstruction algorithm . . . . . . . . . . . . . . . 69

4.11 Transverse spatial resolution of the VRX-CBCT system at different $v r x_{u}$

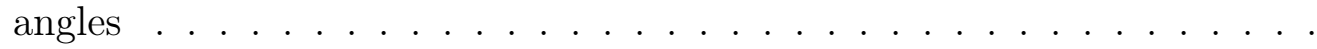

4.12 Transverse spatial resolution of the isotropic VRX-CBCT system at different $v r x_{v u}$ angles . . . . . . . . . . . . . . . . . . . . . .

4.13 Axial spatial resolution of the isotropic VRX-CBCT system at different $\operatorname{vrx}_{v u}$ angles . . . . . . . . . . . . . . . . . 


\section{List of Abbreviations}

$\begin{array}{ll}\text { CBCT } & \text { Cone-beam Computed Tomography } \\ \text { COR } & \text { Center of Rotation } \\ \text { CsI } & \text { Cesium Iodide } \\ \text { CT } & \text { Computed Tomography } \\ \text { FDK } & \text { Feldkemp } \\ \text { FFT } & \text { Fast Fourier Transform } \\ \text { FOV } & \text { Field-of-View } \\ \text { FPD } & \text { Flat Panel Detector } \\ \text { KeV } & \text { Kilo Electronvolt } \\ \text { kVp } & \text { Kilovolt, Peak } \\ \text { LDA } & \text { Linear Detector Array } \\ \text { LSF } & \text { Line Spread Function } \\ \text { MDA } & \text { Multiple Detector Array } \\ \text { MTF } & \text { Modulation Transfer Function } \\ \text { OTF } & \text { Optical Transfer Function } \\ \text { ROI } & \text { Region of Interest } \\ \text { SDD } & \text { Source to Detector Distance } \\ \text { SID } & \text { Source to Isocenter Distance } \\ \text { SNR } & \text { Signal to Noise Ratio } \\ \text { TFT } & \text { Thin-film Transistors } \\ \text { VRX } & \text { Variable Resolution X-ray } \\ \text { VRX-CBCT } & \text { Variable Resolution X-ray Cone-beam Computed Tomography } \\ & \end{array}$




\section{Chapter 1}

\section{Introduction}

In this document, a new Cone-beam Computed Tomography (CBCT) device called the Variable Resolution X-ray Cone-beam CT (VRX-CBCT) is introduced, which attempts to combine the adaptability of the VRX technique with the efficiency of conventional Cone-beam CT. After the implementation, the goal of this project was to analyze the new system for performance and practicality.

There were three objectives in this project:

- Objective 1: Measure the transverse spatial resolution of a VRX-CBCT, consisting of a micro-focal x-ray tube and a CsI-based amorphous silicon Flat Panel Detector (FPD), and test the hypothesis that a higher transverse spatial resolution can be achieved by tilting the FPD about its vertical $v$-axis. In a conventional $\mathrm{CBCT}$, the center line of the $\mathrm{x}$-ray fan beam is designed to be perpendicular to the detector resulting in a maximum spatial resolution limited by the detector's physical characteristics. We investigated the possibility of achieving higher transverse spatial resolution in the VRX-CBCT by tilting the FPD about its vertical axis. The Modulation Transfer Function (MTF) was measured from reconstructed CT images obtained with this configuration and compared to the MTF from images acquired with a similar conventional CBCT.

- Objective 2: Measure the axial spatial resolution of the VRX-CBCT, and test the hypothesis that a higher axial spatial resolution can be achieved using the VRX-CBCT system described in the first objective, by tilting the FPD about its horizontal $u$-axis. The spatial resolution of the central sagittal CT slice was measured experimentally by calculating the MTF of the new VRX-CBCT system at various tilting angles, and the result was compared to the MTF of the system at the conventional CBCT settings.

- Objective 3: Implement a new comprehensive VRX-CBCT calibration and reconstruction algorithm, and measure the transverse and axial spatial resolution of the 3D CT images of isotropic VRX-CBCT, where the FPD can tilt equally about both $u$ - and $v$-axes. By tilting the FPD about both its axes, it was anticipated that a true isotropic 3D CT image could be obtained that would result in higher spatial resolution in transverse and axial directions at the same time when compared to a conventional CBCT. 
A brief background of x-ray imaging and computed tomography is discussed in Chapter 2 of this document. Then in Chapter 3 the methods and materials used to construct an experimental VRX-CBCT, and measure its spatial resolution are described. In Chapter 4, the measurement results are presented, and Chapter 5 contains the conclusions of the work as well as a discussion of the work and suggestions for further improvements to new VRX-CBCT system. 


\section{Chapter 2}

\section{Background}

Computed Tomography $(\mathrm{CT})$ is an x-ray-based method of imaging that employs tomographic principles to create a three-dimensional image of an object. CT imaging has been commonly used in diagnostic medicine since its introduction in 1972. Although the main principle behind computed tomography, the Radon Transform, is almost a century old, it was the introduction of computers in early 1970s that made it practical to create $\mathrm{CT}$ images from the x-ray projection images $[1,2]$. To obtain a $\mathrm{CT}$ image slice, an x-ray tube is rotated $360^{\circ}$ about the patient being scanned while an $\mathrm{x}$-ray detector on the opposite side is collecting the projection image of a single slice of the patient. The projection images obtained from multiple angles are then used to calculate a cross-sectional image of the patient.

In order to thoroughly understand the impact of the Variable Resolution X-ray Cone-beam CT (VRX-CBCT) system, it is important to undestand the underlying principles of x-ray imaging and computed tomography. This chapter is dedicated to explaining these principles.

\subsection{Characteristics of X-rays in Diagnostic Imaging}

$\mathrm{CT}$ imaging uses the attenuation properties of x-rays to generate images. Xrays are a form of radiation that is emitted by the electrons outside of the nucleus of the atom. X-rays are generated by the conversion of electron kinetic energy into electromagnetic radiation [3, 4]. In diagnostic or medical imaging, x-rays are generated by the use of an x-ray tube. An x-ray tube is a vacuum tube that produces $\mathrm{x}$-rays by accelerating electrons (from a cathode) to a high energy and then stopping them abruptly in a target (an anode), most commonly of tungsten, molybdenum or rhodium (Figure 2.1). The spectrum of x-rays generated from an x-ray tube depends on the accelerating voltage and the target material [2].

X-rays can greatly vary in energy (ranging from 1-700 kilo electron volts $(\mathrm{keV})$ ), however for diagnostic imaging, x-ray energies used range from $5-150 \mathrm{keV}$. The range used for diagnostic imaging takes into consideration patient dosage, image sharpness and image contrast $[2,6,7]$. When x-ray energy is too high for a given purpose, the resulting image is not useful because the difference in attenuation/absorption cannot be distinguished, whereas if the x-ray energy is too low, there is generally poor image quality due to high x-ray absorption resulting in low signal-to-noise ratio (SNR). 


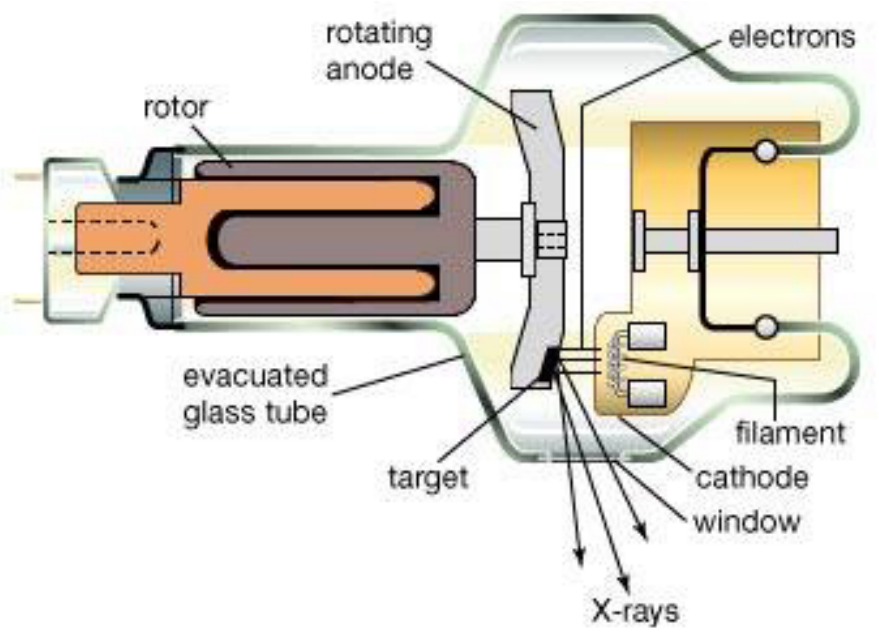

Figure 2.1: Schematic of an x-ray tube.

An x-ray tube works by accelerating electrons and then abruptly stopping them against a target with a high $\mathrm{Z}$-value. The range of $\mathrm{x}$-ray energies created depends on the voltage used for acceleration and the target being utilized. Image from Britannica Student Encyclopaedia, X-ray. [Online]. Available: http://student.britannica.com/elementary/art-66143 [Accessed: 4/12/2009]. 
When electrons hit the anode the majority of their energy is deposited as heat, with only a small fraction, approximately 1\%, creating x-rays [8, 9]. As such, the amount of electrons needed to produce diagnostic levels of x-rays is large, creating large amounts of heat. To handle the heat, the material used for the anodes has high heat capacity and high melting temperature. In addition, rotating anode configurations have been employed as a mechanism to spread out the heat distribution, allowing for greater heat loading, providing higher x-ray output capabilities.

The focal spot is the area of the anode that the electrons hit and from which $\mathrm{x}$ rays are emitted $[10,11]$. The smaller the focal spot, the higher the spatial resolution of the resulting image. In a rotating anode configuration, the focal spot actually moves along a track rather than being stationary. The focal track area is given by:

$$
\text { Focal track area }=2 \pi r \times \Delta r \text {, }
$$

where $\mathrm{r}$ is the radial distance from the track to its center. The heat loading of the anode depends on the rotation speed and the focal spot area, with faster rotation speeds distributing the heat over more of the focal track [12].

When characterizing or evaluating focal spot size, two different measures must be considered: the actual focal spot size and the effective focal spot size. The actual focal spot is the physical area of the focal track that is impacted with electrons from the cathode, while the effective focal spot is the length and width of the focal spot being projected down the central ray in the x-ray field towards the object [2].

There is no difference in the width of the actual focal spot versus the effective focal spot, but the length of the effective focal spot is equal to the following:

$$
\text { Effective focal spot }=\text { Actual focal } \operatorname{spot} \times \sin (\theta) \text {, }
$$

where $\theta$ is the angle of the anode. By angling the anode, in addition to rotating it, the effective focal spot is smaller than the actual focal spot allowing for better axial spatial resolution in the resulting image and larger useful beam. However, the smaller target angle also results in undesired effects such as relatively lower and more variable beam intensity, variable axial spot size, higher intensity modulation, and spot motion [12]. The observed effect of angling the anode is known as the line focus principle.

Variations in effective focal spot size in an image field occur along the anodecathode direction. Towards the anode side, the focal spot shortens, while it lengthens along the cathode side $[13,14,15]$. As the focal spot size (actual and effective) is critical in obtaining images of the highest possible spatial resolution, being able to properly measure this is essential. There are four common methods for measuring focal spot size: pinhole camera, slit camera, star pattern, and bar pattern $[4,13,16]$.

The pinhole camera method employs a very small circular aperture made of a highly attenuating metal positioned on the central axis between the source and the detector. An image is then acquired through the aperture and the focal spot size measured in the resulting image [17]. 
In the slit camera method, a thin slit (versus circle) is made in a sheet of highly attenuating metal. The camera is then positioned above the image receptor with the center of the slit on the central axis. Measuring the width of the slit distribution gives one dimension of the focal spot, while the second dimension is obtained by measuring the distribution with the slit perpendicular to the first slit position [2].

In the star pattern method, a thin plastic disk that contains a radial pattern of lead spokes of diminishing widths is used. The resolving power of the focal spots is determined by measuring the distance between the outermost blur patterns. The effective focal spot size is then estimated from the blur pattern and the known magnification $[2,18]$.

The bar pattern method is the most simplistic. It evaluates focal spot size by determining the effective resolution in a pattern perpendicular and parallel to the cathode-anode axis for a given magnification [16].

Other factors that effect image quality include the heel effect, off focus radiation, and intrinsic filtering. The heel effect is the reduction in x-ray beam intensity towards the anode side of the image field (Figure 2.2). The photons directed towards the anode side of the image field traverse a greater thickness of the anode causing more attenuation [19]. The heel effect decreases as the source-to-image distance increases.

Off focus radiation occurs when electrons hit the anode outside of the focal spot or focal track. These electrons can create a low-intensity x-ray source over the face of the anode. This source of low-intensity x-rays can cause an increase in patient exposure, geometric blurring, and background fog. Off focus radiation is usually reduced by placing a lead collimator near the output of the x-ray tube to intercept the stray x-rays [20].

Inherent filtration preferentially reduces the lower energies in the output energy profile that reduces x-ray absorption and thus the absorbed dose within the patient but lowers the signal as well.

\subsection{How X-rays Are Detected}

The photons generated from an x-ray tube must be detected to create the image. Currently, there are three main types of detection methods: film-screens, image intensifiers, and digital detectors.

\subsubsection{Film Screen Detector System}

Film-screen detector systems are primarily used in projection radiography, the acquisition of a 2D image of a 3D object, and consist of a cassette containing an intensifying screen and film $[21,22,23]$. The cassette has two main functions: provide

a light tight environment for the film, and create physical contact between the film and 


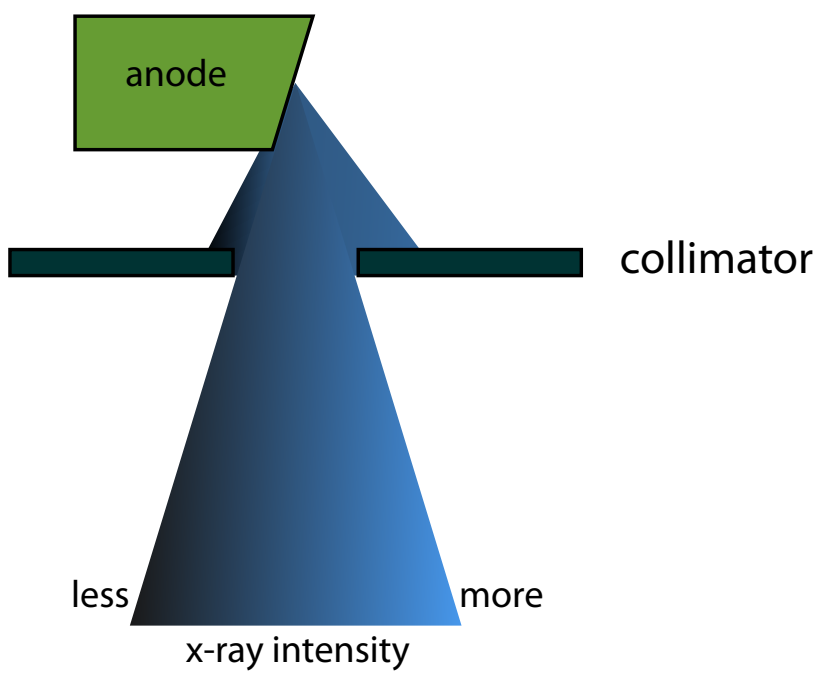

Figure 2.2: Heel effect in x-ray tubes.

$\mathrm{X}$-rays emitted on the anode side traverse a greater thickness of target material and are therefore more attenuated than those emitted along the cathode side. 
the intensifying screen(s). The light-tight environment prevents ambient light from exposing the film, while the physical contact reduces imaging artifacts and is essential for good image quality. Film provides very high resolution; however, it is relatively insensitive to detecting x-rays. It is therefore almost always used in conjunction with one or two intensifying screens. The intensifying screens are comprised of rare earth minerals and phosphor which convert x-rays to visible light or low electromagnetic energies that sensitizes the film $[2,21]$.

\subsubsection{Image Intensifiers}

Image intensifiers are analog devices that convert the acquired x-ray image into one visible on a video screen. This device is made of a vacuum tube with a large circular input surface coated on the inside with cesium iodide (CsI). When hit by $\mathrm{x}$-rays, the material phosphors which causes the photocathode adjacent to it to emit electrons [24]. These electrons are then focused, using electron lenses inside the intensifier, to a smaller output screen coated with phosphorescent materials. The optical image from the output screen can then be recorded via a camera and displayed $[25,26]$.

\subsubsection{Digital Detectors}

Digital devices known as array detectors are becoming more common in all realms of diagnostic imaging. These devices are made of discrete pixelated detectors which can either work indirectly by using photo detectors that detect light emitted from a scintillator, or directly by capturing the electrons produced when the x-rays hit the detector [27]. Direct detectors do not tend to experience the blurring or spreading effect caused by phosphorescent scintillators or film screens since the detectors are activated directly by x-ray photons. There are two main types of array-based detectors: linear array detectors and flat-panel detectors [27, 28].

\subsubsection{Linear Detector Arrays}

A linear detector array (LDA) is a one dimensional array of solid-state detectors arranged in a single row. These detectors are usually suitable for conventional $2 \mathrm{D}$ CT systems, where a single CT slice is obtained in each scan iteration. In LDAs, the scintillator emits visible light after being struck by x-ray photons. The light photons are then detected by the photodiode underneath the scintillator crystal, and an electric signal proportional to the intensity of the light is generated. Typical scintillator materials for linear detector arrays used in CT are cadmium tungstate $\left(\mathrm{CdWO}_{4}\right)$ and gadolinium oxysulfide $\left(\mathrm{Gd}_{2} \mathrm{O}_{2} \mathrm{~S}\right)$. The size of each detector cell in a LDA varies depending on the application. In CT, this size is typically $1.0 \mathrm{~mm} \times 15 \mathrm{~mm}$, 
however it is possible to arrange several LDAs of smaller height (typically $1.5 \mathrm{~mm}$ ) in multiple rows to create a multiple detector array (MDA). The detector height in MDAs are determined at the detector level instead of by the collimator.

One important characteristic of LDAs is the existence of lead barriers between each two adjacent detector cells. These barriers significantly reduce the number of x-ray quanta that pass across the cell chambers. In addition, aluminum oxide reflector layers confine the light photons generated in each cell chamber and direct them to the corresponding detector photodiodes. This feature in the structure of LDAs makes this type of detector suitable in not only conventional CT systems, but also other applications where the x-ray reaches the detector surface at an oblique angle. An example of such situations is a variable resolution x-ray CT system (VRXCT) $[29,30]$, where the $\mathrm{x}$-ray detector is tilted to increase the spatial resolution of the object being scanned.

\subsubsection{Flat Panel Detectors}

Flat panel detectors (FPDs) fall into one of two categories, direct or indirect detectors. Direct FPDs are made by placing a photoconducting material, usually amorphous selenium (a-Se) on top of an active matrix array of charge-collecting electrodes and thin-film transistors (TFT). In direct FPDs, electrons released in the detector layer from x-ray interactions form the image directly. There is a negative voltage that is applied to the front surface of the detector causing the detector elements to be held positive in relation to the top electrode [31]. During exposure, x-rays liberate electrons in the selenium layer that then migrate under the influence of the applied electric field and collect on the detector elements. Because of the ability to change the electrical field and direct the paths of the electrons, the spatial resolution of direct FPDs is primarily limited by the dimensions of the detector elements.

Indirect FPDs convert incident x-rays into light which is then detected by an active matrix array of photodiodes and TFTs. This system is analogous to a filmscreen detector system except that the film is replaced by the electronic sensor. The term indirect comes from the fact that x-rays are first converted to light photons and then to electrical charges [32, 33]. An indirect FPD consists of a phosphor layer or structured scintillator, typically cesium iodide doped with thallium (CsI:Tl) or gadolinium oxysulfide doped with terbium $\left(\mathrm{Gd}_{2} \mathrm{O}_{2} \mathrm{~S}\right.$ : Tb), in contact with a thinfilm transistor active-matrix array (TFT). The intensity of the light emitted from a particular point on the phosphor of an indirect FPD is a measure of the intensity of the x-ray beam incident on the surface of the detector at that point. Each pixel in the active matrix has a photosensitive element that generates an electrical charge proportional to the light intensity emitted from the phosphor in the region near the pixel. The magnitude of the signal charge is proportional to the intensity of the incident x-ray beam [2]. 
Since the scintillator in FPDs is a continuous crystal, in contrast to the scintillator crystals in LDAs that are separated by layers of lead and aluminum oxide reflective surfaces (Figure 2.3), the light photons generated along the trajectory line of x-ray photons may reach multiple detectors, creating blurring in the final image obtained from the detector that usually depends on the size of each detector cell, as well as the thickness of the scintillator layer. This blurring effect increases when the x-ray photon hits the surface of the detector at an angle, therefore FPDs are traditionally used in applications where the x-ray beam is perpendicular to the imaging surface, such as in projection radiography.

\subsection{Current Uses of X-rays in Diagnostic Imaging}

Since their discovery, x-rays have been used in the field of medicine for diagnostics purposes and treatment. Today, there are many different imaging modalities that utilize x-rays for a wide array of uses. Below are a few of these modalities described in more detail.

\subsubsection{Projection Radiography}

Projection radiography is a two-dimensional image created by exposing an object to x-rays, the underlying concept in CT imaging [34]. Projection radiography is one of the most common uses of x-rays due to its simplicity, high diagnostic yield and low cost. Projection radiography was, and to some extent still is, performed using a filmscreen detector system, however, newer systems are designed using digital detectors. Objects with high x-ray attenuation such as bones and lungs are imaged very well with this method $[8,34]$.

\subsubsection{Mammography}

Mammography is the technique commonly used to screen women for breast cancer as it is designed to provide high-resolution images of soft tissues, such as the breast. The x-rays used in mammography are softer than in other applications, often employing a molybdenum anode rather than a tungsten anode, generating $\mathrm{x}$-ray energies in the range of $15-30 \mathrm{keV}[8,10,35]$.

\subsubsection{Fluoroscopy}

Fluoroscopy is a technique where lower quality digital projection radiographs

are taken over time. It is often used to view the movement of a contrast agent as it travels along a path, such as in the intestines, or as a guide during a procedure, 


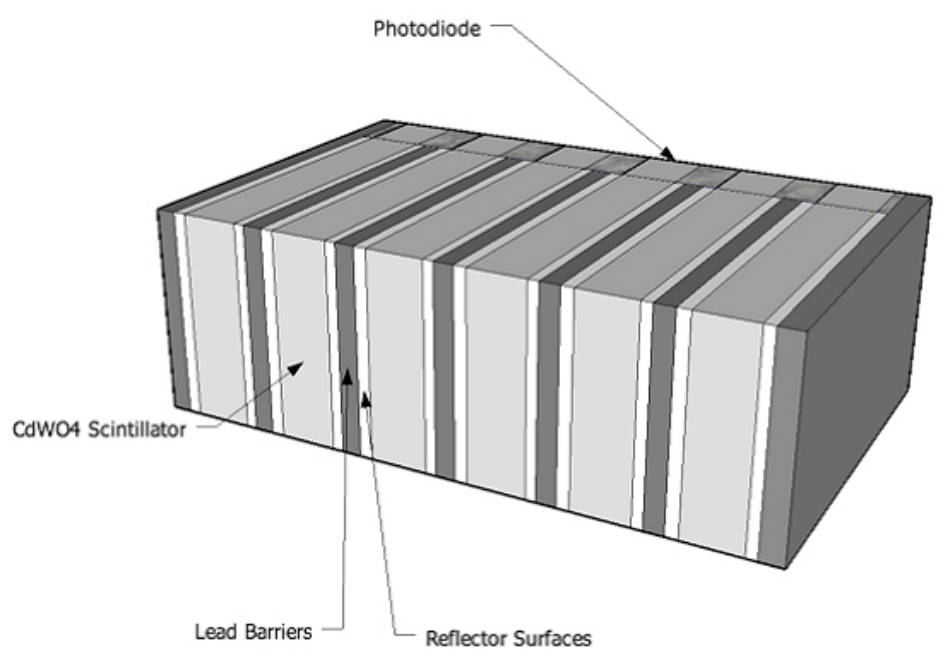

(a)

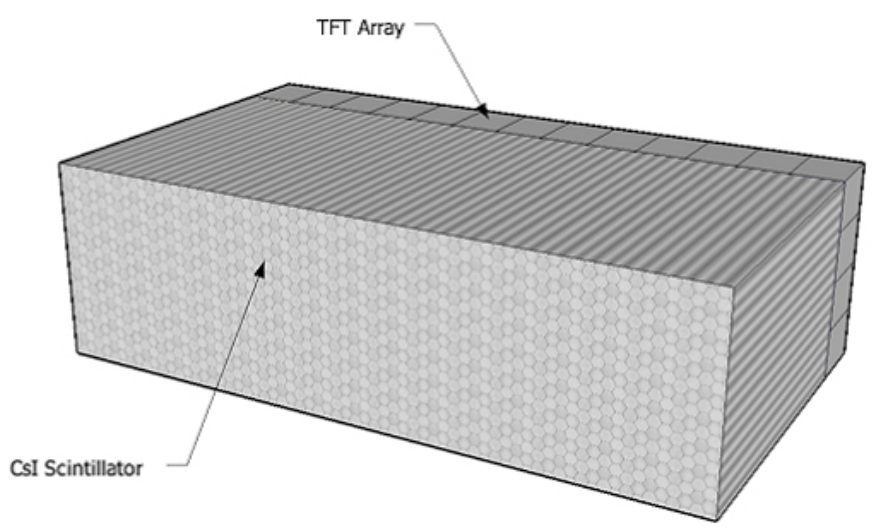

(b)

Figure 2.3: Comparison of linear array and flat panel detectors.

In linear array detectors (a) the lead barriers and aluminum oxide reflective surfaces minimize the effect of cell cross-talk, whereas in flat panel detectors (b) the x-ray and light photons can travel in the scintillator causing signal blurring. Reprinted with permission from B. Dahi, G. S. Keyes, D. A. Rendon, and F. A. DiBianca, "Performance analysis of a CsI-based flat panel detector in a cone beam variable resolution x-ray system," SPIE - The International Society for Optical Engineering, vol. 6510, pp. 65 104B-1 - 65 104B-8, 2007. 
such as angioplasty. A fluoroscopy system is usually housed on a C-arm so that it can rotate all the way around the patient taking images at all angles [36, 37].

When a fluoroscopy system is used to view the cardiovascular system it is called an angiography. During an angioplasty procedure, a contrast agent is imaged as it is injected and as it travels through he blood stream. The contrast agent is usually iodine-based and has a high density, which creates good contrast between the blood and vasculature, which are not very dense. Angiography is used in a variety of procedures including the placement of catheters, and locating blockages, leaks, or aneurysms [37].

Another common use of fluoroscopy is to image the digestive tract, using a material opaque to x-rays. This material can be a variety of things, but is usually barium sulfate. Barium sulfate appears white on x-rays, and can be followed easily as it travels through the gastrointestinal system. In addition, another contrast material, such as air, may be used to examine further along the path that is visualized by the barium sulfate [36].

\subsubsection{Tomosynthesis}

Digital tomosynthesis produces slice images from a conventional x-ray system by taking a discrete number of images, usually about 10 or 12 , over a small rotational angle of about 40 degrees $[8,27]$. The acquisition of data in this manner provides an incomplete data set, but by shifting and adding the projected radiographs the full data set can be obtained [8].

Tomosynthesis does not allow the narrow slices that are obtainable in CT imaging, but by using high-resolution detectors, a high in-plane resolution can be achieved. Because of the achievable resolution, tomosynthesis is currently being aimed as an alternative to mammography imaging for breast cancer screenings. The use of tomosynthesis for breast imaging does not require the level of pressure needed in mammographic imaging, and usually takes less than 10 seconds for the acquisition of the data set [27].

Current advances in tomosynthesis imaging are focusing on the reconstruction algorithms that are used to align the planes as well as to minimize residual blur from out-of-plan structures. The reconstruction algorithms used for tomosynthesis are very different from conventional CT, as they use an iterative algorithm based on expectation maximization compared to the traditional filtered back-projection algorithm used in conventional CT. 


\subsection{Computed Tomography Imaging}

\subsubsection{Conventional CT}

Computed Tomography (CT) technology has advanced dramatically since the first such device was developed over three decades ago. To date there have been seven different generations of clinical CT scanners, with each generation improving and extending the capabilities of the technology [38]. Most of the improvements over the years focused on lowering exposure time and patient dose, as well as mechanical design improvements to accommodate such enhancements. The new generations of clinical CT scanners are capable of scanning the entire average human body in a few seconds with moderate spatial resolution. The voxel size in these devices reach to about $0.3 \mathrm{~mm} \times 0.3 \mathrm{~mm} \times 0.5 \mathrm{~mm}$ [38], which corresponds to approximately $3 \mathrm{cy} / \mathrm{mm}$ in-plane resolution. Although this resolution is enough for most clinical applications, where the region to be scanned is about $40-50 \mathrm{~cm}$, it is not sufficient for scanning smaller objects. In other words, the spatial resolution of the final CT image does not increase as the size of the object being scanned decreases in current clinical CT scanners [2, 39].

In the first generation of $\mathrm{CT}$ scanners, a rotate/translate pencil beam system was used (Figure 2.4a). The scanner had a single x-ray source and single detector for each slice and used parallel ray geometry. The system had a field of view of $24 \mathrm{~cm}$ and used 160 parallel rays for each projection. An image was acquired by translating across the field of view, and then rotating and repeating the process. The complete data set acquired a full 180 degrees at one degree intervals [40, 41].

The most significant change between the first and second generations of CT scanners was the inclusion of multiple detectors (Figure 2.4b). The second generation of CT scanners employed a linear array of 30 detectors while maintaining a single source. To utilize the linear array, a fan beam geometry was introduced. The fan beam only had an angle of ten degrees, however it had a high rate of scatter rejection increasing signal. The second generation still acquired a data set using the rotate/translate system, however at ten degree intervals rather than one degree intervals. The second generation $\mathrm{CT}$ was significantly more efficient and faster than the previous generation $[38,42]$.

By the third generation of CT scanners, it was noticed that the rotate/translate system reduced the efficiency of the system, therefore in the third generation CT scanners a rotate/rotate system was used instead (Figure 2.4c). In this system, the detector and x-ray tube are mechanically connected and rotate around the patient. The rotate/rotate description of the system comes from the simultaneous rotation of both the x-ray tube and detector array. The third generation CT scanners also greatly increased the number of detectors to over 800 and increased the angle of the fan beam such that a large arc is formed designed to cover the entire patient. In this set-up however, there was a high incidence of ring artifacts, requiring the use of high performance detectors, and the system in general was more sensitive to aliasing than previous generations [23, 38]. 


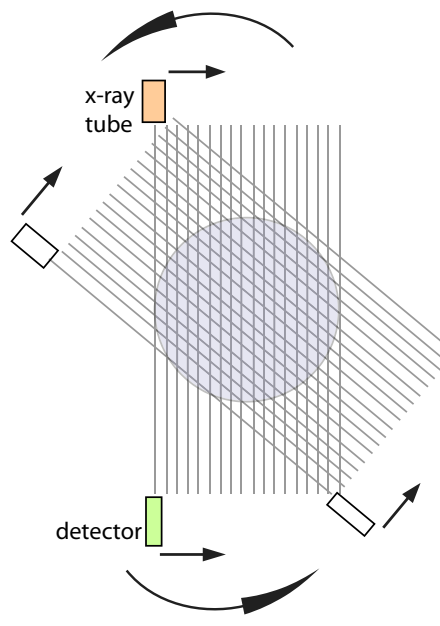

(a)

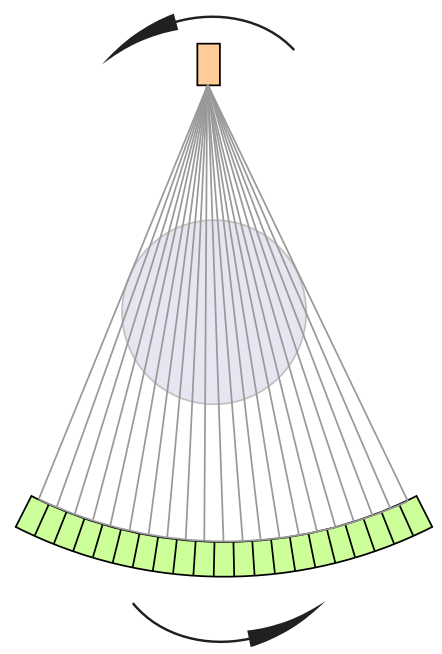

(c)

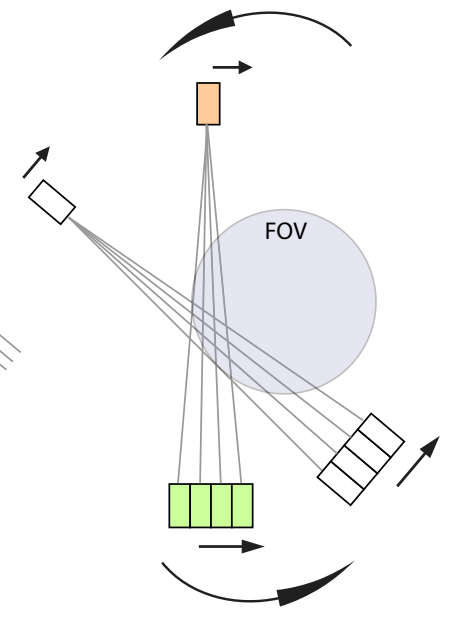

(b)

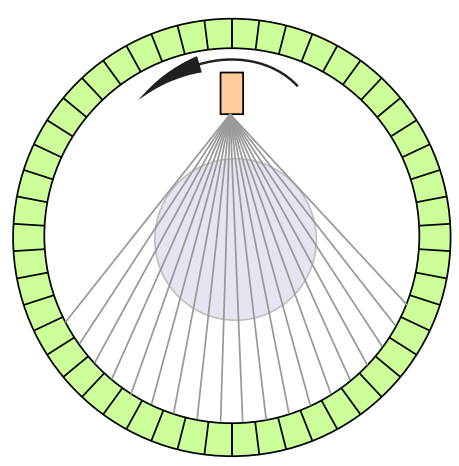

(d)

Figure 2.4: Generations of clinical CT scanners.

Over the past 40 years, there have been many advances in CT systems. Most of the advances have centered around significant changes in hardware. The first generation (a) employed a single source and a single detector cell, while the second generation (b) employed a single source with multiple x-ray detectors. The third generation (c) of CT scanners began using fan-beam source to obtain a cross-section of an entire patient, while the fourth generation (d) uses a detector ring and a rotating $\mathrm{x}$-ray tube. 
The fourth generation of CT scanners (Figure 2.4d) was designed to primarily to reduce the ring artifacts that were a problem in the third generation scanners. Fourth generation scanners employed a rotate/stationary design, meaning the x-ray tube rotates while the detectors remain stationary. Additionally, the number of detectors used increased to about 4800 as they had to span 360 degrees to completely surround the patient $[38,43,44]$.

The fifth generation of CT scanners was designed for cardiac imaging. In this system, both the x-ray source and detectors are stationary. In place of a traditional $\mathrm{x}$-ray tube, a vacuum pump was placed behind the scanner that generated electrons $[2,38]$. The electrons were then steered towards a tungsten arc around the patient to generate the x-rays. In this set-up it was possible to achieve scan times of $50 \mathrm{~ms}$, which is adaquate for imaging the beating heart.

The sixth and seventh generations of CT scanners are improvements to the third generation of CT scanners [2,38]. The sixth generation introduced helical scanning while the seventh generation introduced multiple detector arrays. In helical scanning, the x-ray tube and detectors rotate while the patient is translated through. This creates a helical path around the patient that can complete a scan of an abdomen in approximately 30 seconds. The inclusion of multiple detectors in the seventh generation was a major shift in the technology. Multiple detector arrays allow for increases in collimator spacing capturing more of the generated x-rays. Multiple detector arrays also allow for more control over slice thickness and spatial resolution $[2,38]$.

In $\mathrm{CT}$ imaging the time it takes to scan the object, scan time, is very important and must be taken into consideration when developing the system. When the scan object is stationary, or has no inherent movement to it, scan times can be longer and focus more on resolution and overall image quality. However, when the scan object is actively moving, as in the case of the torso with the lungs, the scan time is limited by the ability of the patient to hold their breath to stop the movement. In the case of the heart, the scan time must be very fast as its movement cannot be limited or controlled. Nowadays in cardiac CT imaging, the concept of gating is used to overcome the motion problem in order to complete a scan. In gating, the movement or beating of the heart is monitored by the CT system, and the scan is performed at intermittent times when the heart is stationary in-between beats.

\subsubsection{Cone-beam CT}

Cone-beam CT is the $2 \mathrm{D}$ acquisition projection data where the x-rays are emitted from the source in a cone shape rather than in a single plane [2]. Clinically, conebeam CT is used in the helical CT where the x-ray tube and detectors rotate while the patient is translated through. This set-up reduces the radiation dose the patient is exposed to while decreasing scan time. The reconstruction of the projection data is done using back-projection-based, cone-beam reconstruction algorithm that takes the 
$\mathrm{x}$-ray beam divergence in both spatial dimensions into account. The main limitation of cone-beam CT is x-ray scattering, which reduces image quality, and the mathematical complexity of its reconstruction $[8,45,46]$.

\subsubsection{Micro-CT Imaging}

The introduction of micro-CT imaging of small animals has revolutionized biomedical research and opened the door to a new wave of small animal imaging modalities. There is micro-CT imaging equipment in almost every research center around the nation. These systems allow for repeated measurements in animal disease models eliminating the need to sacrifice a large number of animals and reducing the variability between different animals used at different time points. When coupled with positron emission or single photon emission imaging these systems allow for molecular imaging of disease progression and for the study of drug bio-distribution and effect in live animals $[40,47,48,49]$.

Micro-CT scanners consist of a microfocus x-ray tube, with a focal-spot size between 10-100 $\mu \mathrm{m}$, and a high-resolution (pixel spacing $<50 \mu \mathrm{m}$ ) detector which is a scintillating screen optically connected to a charge-coupled camera $[50,51]$. Typically micro-CT scanners use a cone-beam data collection and reconstruction method. Cone-beam collection has the advantage of faster volumetric acquisition. One such reconstruction which is an approximation of 3D filtered backprojection is the Feldkamp (or FDK) algorithm [45].

The FOV of micro-CT scanners ranges from about 50 to $100 \mathrm{~mm}$ and a usual configuration in these scanners is one in which the gantry rotates around the animal. Fixing the animal while rotating the gantry helps avoid any tissue distortion in the final reconstruction. The scanners are optimized for minimal radiation dose to the animal to allow for longitudinal measurements carried on the same animal over time $[50,51]$.

Micro-CT scanners have also been developed for in vitro imaging to scan small industrial or tissue specimens. In the in vitro systems the specimen is made to rotate around its vertical axis with the x-ray tube and detector stationary [52]. Because there is no requirement to minimize radiation dose, the scanners are optimized for maximum spatial resolution. This resolution can almost reach that of microscopy, approximately 10-50 $\mu \mathrm{m}$, with a scan time of 10-30 min.

The use of synchrotron x-ray source can dramatically improve the resolution of the micro-CT scanners [51]. These scanners can achieve a spatial resolution of $1 \mu \mathrm{m}$ or higher, approaching the subcellular imaging limit [53]. These scanners can not use cone-beam reconstruction because of their parallel x-ray beam configuration [54]. 


\subsection{Variable Resolution X-ray CT System}

Detector resolution is the main limiting factor of the spatial resolution of conventional CT scanners which employ detectors composed of discrete cells. Detector resolution is determined, among other things, by the physical size of the detector cells. A typical cell size of $250 \mu \mathrm{m}$ results in a maximum spatial resolution of $2.0 \mathrm{lp} / \mathrm{mm}$. Given the large FOV $(40-50 \mathrm{~cm})$ of clinical machines and the number of detectors used to cover such a large area, reducing the detector size would be an expensive alternative. Furthermore, reducing the physical cell size would also result in a reduction of resolution resulting from electron cloud overlap (Figure 2.5a) [29, 55, 56].

An alternative approach would be to employ the principle of "projective compression" in which the detector is constructed such that the detector cells appear smaller and closer together in projection, as viewed from the x-ray source. This could be achieved either by detector angulation (Figure 2.5b) or by "stairstepping" (Figure 2.5c). A straightforward application of this principle is to angulate the detector with respect to the x-ray beam which would improve spatial resolution by a factor of $(\sin \theta)^{-1}$, where $\theta$ is the angle between the detector surface and the x-ray beam direction. There are two limits to this angulation increase in resolution; a) as the angle increases x-ray signal intensity decreases, and b) as the angle increases x-ray reflection increases. However, even with these limitations, resolution can be increased by a factor of over 800 . Projective compression can also be achieved by arranging the detector cells in a stepwise fashion in which reducing the cell-to-cell offset increases the resolution. The main limitations of this geometry are signal intensity and manufacturing complexity $[29,55,56]$.

Projective compression also allows for a change in detector projection dependent on the FOV in which the FOV is tailored to the object size by changing either the cell-to-cell offset or the detector angulation. This way a maximum resolution can be achieved for each object size, hence the term given to this procedure "variable resolution x-ray" detection technique.

Several VRX configurations have been studied: single-arm, dual-arm, and fourarm VRX detectors. In the single-arm configuration, the VRX detector includes one scintillator-photodetector array (one arm) which can rotate around a pivotal point (Figure 2.6a). The detector's spatial resolution can be varied by tilting the arm.

The dual-arm VRX detector is made of two scintillator-photodetector arrays that can tilt around a common pivotal point. The arms can be arranged either symmetrically (Figure 2.6b) or asymmetrically (Figure 2.6c) around the detector centerline, which is the line connecting the x-ray source and the detector vertex $[29,57,58]$. In the symmetrical (or "normal") mode, both arms provide the same increase in spatial resolution and are always angulated equally to cover the full FOV. In the asymmetrical (or "target") mode, only one arm (low-resolution arm) is angulated according to the FOV. The other arm (high-resolution arm) is set at a smaller angle with the detector centerline and covers just a small target region inside the FOV during a CT scan. This latter region is imaged with higher spatial resolution than the rest of 


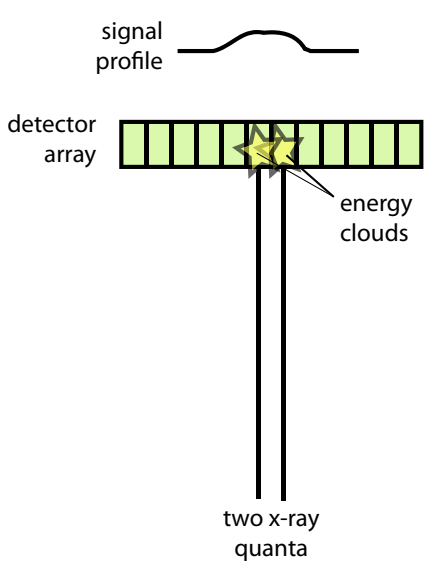

(a)

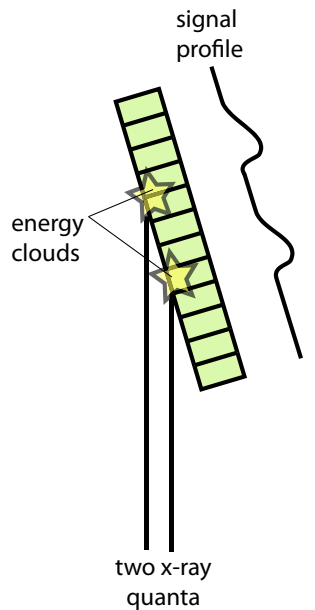

(b)

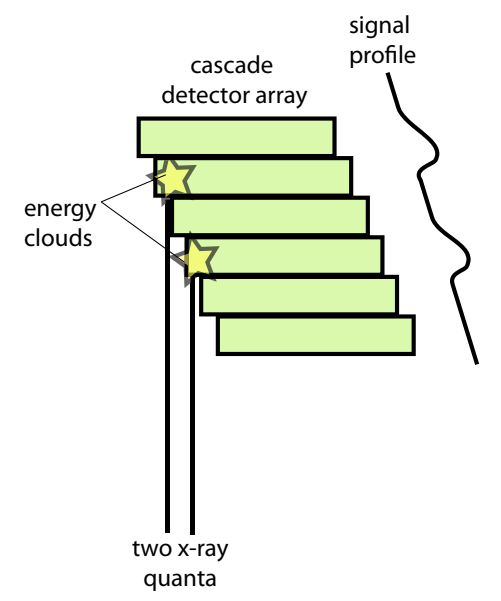

(c)

Figure 2.5: X-ray detector configurations.

$\mathrm{X}$-ray detector configurations that have been evaluated in the VRX-CT system. A) The conventional heads-on approach to the detector array. B) The angled detector from the original VRX set-up. C) The newest stair-step detector approach. 


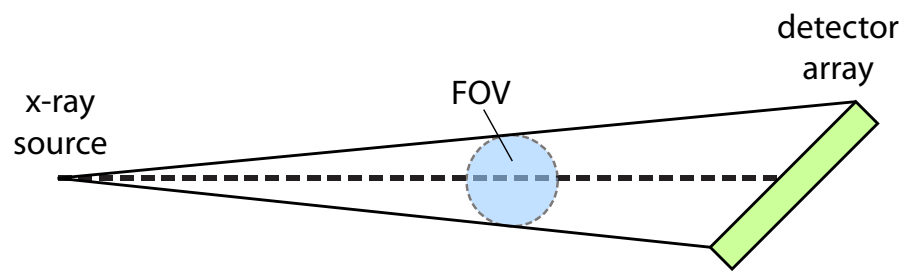

(a)

Single-arm

VRX CT

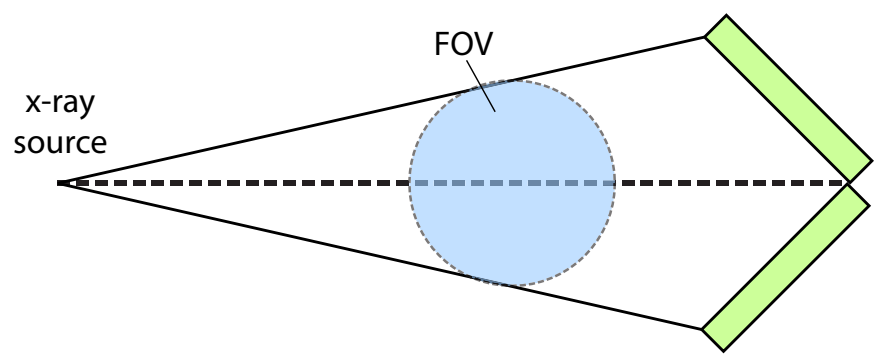

(b)

Dual-arm

VRX CT

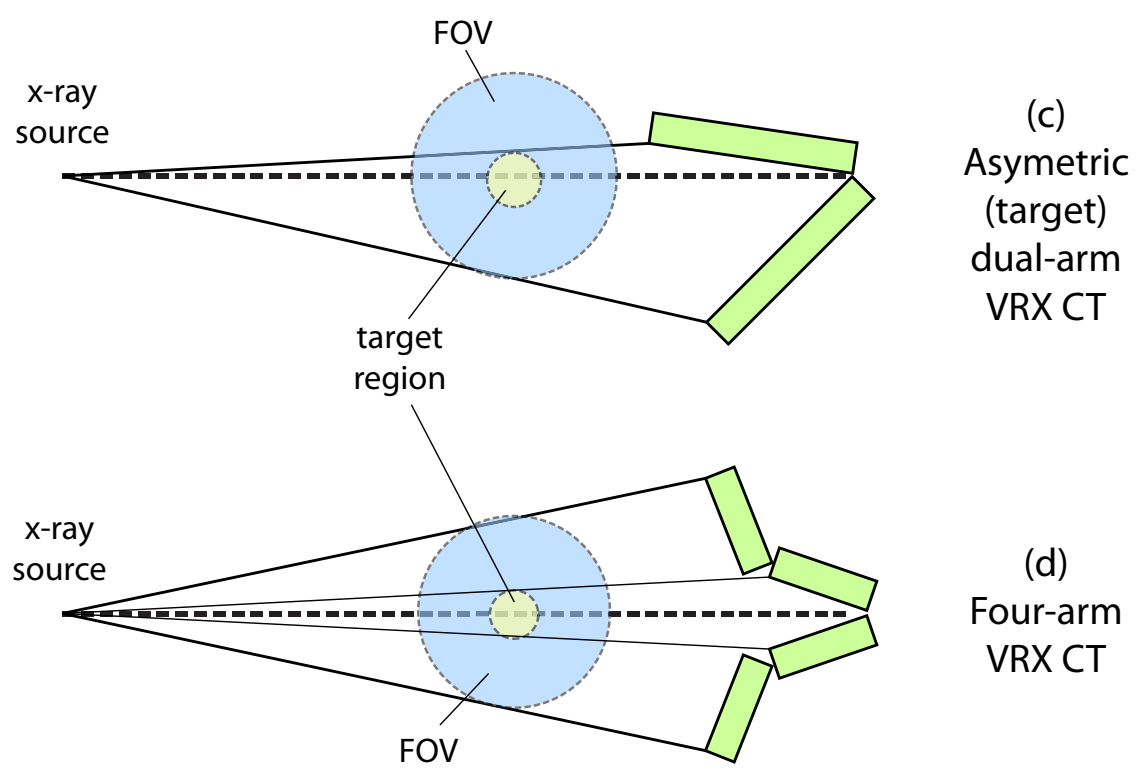

Figure 2.6: Generational configurations of the VRX-CT system.

Graphical depiction of the different configurations the VRX-CT system has taken through its multiple generations of development. 
the object [57, 59, 60]. Thus, the asymmetrical mode of the dual-arm VRX detector allows "target imaging" and offers an additional resolution improvement for the target, compared with the basic VRX detection technique. The asymmetrical mode can use several arrangements of the detector arms. Those arrangements differ mainly by how much of the FOV and target is covered in a single projection by the low- and high-resolution arms, respectively. In both symmetrical and asymmetrical modes, the dual-arm VRX detector can be easily converted to the single-arm configuration by opening the arms to form a 180-deg angle between each other [58, 59, 61].

The four-arm VRX detector includes two inner and two outer arms (Figure 2.6d). The inner arms are oriented and act similarly to the dual-arm configuration. The outer arms are placed at the ends of the inner arms, at a larger angle, relative to the detector centerline, than that of the inner arms and do not have any fixed pivotal points. While moving these arms, care is taken to avoid blocking any x-ray path toward the inner arms. Both pairs of the arms are usually positioned symmetrically around the detector centerline, so that the inner arms cover a small target region, while the outer arms scan the rest of the FOV. Due to larger angulation (smaller angles with the detector centerline) for the inner arms compared with the outer arms, the target is imaged with higher spatial resolution than the rest of the object.

While each configuration has its advantages and limitations [58], the main advantage of the four-arm configuration of the VRX detector is that it can easily be converted to the dual-arm or single-arm configuration. Also because of the inherent left-right symmetry of this configuration it is the most suitable for target imaging. The limitation of this configuration when compared to the single or dual-arm one is that it shows larger variations in the detector and system performance from one end of the detector to the other, higher x-ray scatter between the inner arms, and more gaps or discontinuities in the detector.

\subsection{Modulation Transfer Function}

The modulation transfer function (MTF) is a complete description of the resolution properties of an imaging system. An MTF curve is a plot of an imaging system's modulation versus spatial frequency, where the modulation is defined as the output contrast normalized to the input contrast. The MTF represents the fraction of an object's contrast that is recorded by the imaging system as a function of the spatial frequencies of the object $[4,13,62]$.

In practice, the MTF of an imaging system is calculated by taking the fourier transform of the line spread function (LSF) of the system.

$$
\mathrm{MTF}=|\mathcal{F}\{\operatorname{LSF}(x)\}|
$$

The LSF is measured by imaging a narrow slit to collimate the x-ray beam to a narrow line. To obtain the LSF for a tomographic system, a thin sheet of material, 
usually copper, is used. From the function, as the LSF of a system gets broader or the resolution decreases, the MTF curve reaches zero modulation more quickly $[13,30,62]$.

As the MTF is a description of the system, the overall MTF will depend on the individual components of the system. In a CT system, the main components that contribute to spatial resolution are x-ray image formation, x-ray detection, analogto-digital conversion, and image reconstruction [63]. When x-rays are emitted, the sharpness of the image they can create is inherently limited by the focal spot size and magnification and this will create an unsharpness in the formed image. Once the image is formed, it must be detected. The main effect that the detector has on resolution is blurring due to x-ray scatter, light diffusion, and electron spreading. The structure and quality of $\mathrm{x}$-ray detection have a significant effect on the overall MTF of the imaging system. Once the x-rays are detected, they must be converted into a digital signal, relating photon count to a digital signal. This conversion can introduce many effects including sampling aperture (defined by the size and shape of the detector cells) and sampling distance (the spatial interval at which the image is sampled). The last major component in an imaging system that effects the resolution of that system is image reconstruction. In most CT scanners, images are generated by mathematically reversing the acquisition geometry, most commonly through filtered backprojection. The accuracy of the reconstruction is often a major limiting factor in system resolution as many artifacts can be introduced [4, 25, 41, 62].

There are many factors that can contribute to reduced resolution in an imaging system. Some of these factors can be addressed, such as the accuracy of image reconstruction and the collection of the x-rays during image formation, while others are more difficult to control. As new CT systems are developed, spatial resolution is a primary objective, and as such, an emphasis of this work is to improve the spatial resolution of the VRX-CT system by enhancing the VRX effect and introducing new calibration and reconstruction algorithms. 


\section{Chapter 3}

\section{Variable Resolution X-ray Cone-beam CT (VRX-CBCT)}

\subsection{Introduction}

The original VRX CT scanners demonstrated significant improvement in spatial resolution of small objects $[55,30]$. The two-arm and four-arm VRX CT scanners helped with the asymmetry in acquiring the projection data as well as achieving higher resolution for the center of the CT reconstructed image, in the latter case. However all those variations of VRX CT scanners have one major limitation: they provide only a single slice of CT image per scan. For acquiring multiple slices per scan in a CT system, a few options are available.

An intuitive way of creating a multi-slice CT image is to replace the single linear detector array (LDA) with a multiple detector array (MDA). A multiple detector array is a collection of several linear detector arrays that are held tightly together to form a panel. While a traditional single layer CT usually uses wide solid state detector's in its LDA (about $15 \mathrm{~mm}$ for example), narrower solid state detectors are usually used in MDAs (1.25 mm for one example of a 4-slice CT detector). The slice thickness can then be adjusted by binning the adjacent detectors together to reach a desired total cell width [2] therefore creating 4 slices of $1.25 \mathrm{~mm}, 2.50 \mathrm{~mm}, 3.75 \mathrm{~mm}$, or $5.00 \mathrm{~mm}$. However, the minimum slice thickness and the maximum number of slices are both limited by the detector cell width and the number of LDAs in the detector panel. In order to get more slices, the system needs to move the subject along the axial direction and repeat the acquisition process, or it uses a continuous helical motion for the detector relative to the subject.

In a helical CT scanner, the subject moves steadily along the axial direction i.e. perpendicular to the CT planes - while the x-ray tube and the detector rotate at a constant speed. The final result of this geometry is equivalent to that of a system where the x-ray source and the detector move in a helical path around the stationary subject. Theoretically the axial resolution of the helical CT scanner can be increased by reducing the speed of the moving subject along the axial direction, hence reducing the helical pitch. However every point on a planar slice of CT image is always an interpolation of two points in the helical space. Such interpolation affects the true magnitude of spatial resolution increase. As with the non-helical CT scanners, the 
transverse spatial resolution in helical CT systems is limited by the detector width and the x-ray source focal spot size.

Based on our experience with the original single arm VRX CT for improving inplane resolution, we believed that it would be possible to use the VRX technique to design a volumetric CT scanner with adjustable transverse and axial spatial resolution $[64,65]$. The proposed system should be able to demonstrate not only improvement in spatial resolution of the final 3D CT image, but also have an intrinsic ability to create isotropic CT images.

\subsection{Design and Methods}

Although there may be several alternatives to our design, we decided to set up our new device as a Cone-beam CT (CBCT) using a CsI-based indirect flat panel detector (FPD) and a micro-focal x-ray tube. The object being scanned is mounted on a precision rotary table while both the detector and the x-ray tube are stationary. The reconstructed images using this geometry are essentially equivalent to the ones from conventional Cone-beam CT systems. This test-bed configuration enables us to avoid developing a complex gantry system for our experimental CBCT scanner.

\subsubsection{Basic Components}

\subsubsection{Flat Panel Detector}

The FPD in our experiments was a PaxScan 2020 (Varian Medical Systems, Salt Lake City, UT, US). Table 3.1 shows the specifications of the detector. According to the manufacturer's website, the PaxScan 2020 has been specifically designed for cardio-vascular diagnostic imaging equipment and mobile C-arms [66]. It is capable of taking sequences of images at a maximum rate of $30 \mathrm{fps}$ (frames per second) at $1024 \times 1024$ intrinsic resolution. The CsI scintillator and the noise management techniques used in this device make it a highly sensitive x-ray detector that works relatively well when coupled with our low-power micro-focal x-ray tube.

\subsubsection{Micro-focal X-ray Tube}

The x-ray tube used in our experiment was a SRI SB-80-250 (Source-Ray Inc., Bohemia, NY, US) with maximum power of $20 \mathrm{~W}$ and nominal focal spot size of $36 \mu \mathrm{m}(\mathrm{W}) \times 65 \mu \mathrm{m}(\mathrm{H})$. Table 3.2 describes the complete list of specifications for the tube. This tube is capable of providing a continuous x-ray beam of $250 \mu \mathrm{A}$ intensity at a maximum $80 \mathrm{kVp}$ energy level. When positioned at $1 \mathrm{~m}$ distance from the PaxScan 2020 FPD, we are able to achieve an average signal to noise ratio (SNR) of $54 \mathrm{~dB}$ with the FPD set to $2 \times 2$ binning at $15 \mathrm{fps}$. 
Table 3.1: Flat panel detector specifications.

\begin{tabular}{llll}
\hline Parameter & Value & Parameter & Value \\
\hline active area & $198 \mathrm{~mm} \times 198 \mathrm{~mm}$ & frame rate & $30 \mathrm{fps}$ \\
pixel pitch & $194 \mu \mathrm{m}$ & maximum energy & $150 \mathrm{kVp}$ \\
active matrix & $1024 \times 1024$ & scintillator material & CsI \\
fill factor & $70 \%$ & scintillator thickness & $500 \mu \mathrm{m}$ \\
\hline
\end{tabular}

Table 3.2: X-ray tube specifications.

\begin{tabular}{llll}
\hline Parameter & Specs & Parameter & Specs \\
\hline target material & Tungsten & tube voltage range & $35-80 \mathrm{kVp}$ \\
focal spot size & $36 \mu \mathrm{m}(\mathrm{W}) \times 65 \mu \mathrm{m}(\mathrm{H})$ & tube current range & $10-250 \mu \mathrm{A}$ \\
intrinsic filteration & $1.8 \mathrm{~mm} \mathrm{Al}$ & & \\
\hline
\end{tabular}

\subsubsection{Rotary Table}

A Techno-Isel Model 5 stepper-motor rotary table (Techno-Isel, New Hyde Park, NY, US) was placed between the FPD and the x-ray tube. The rotary device is capable of moving at angular increments of $4.5 \times 10^{-3}$ degrees $\left(7.85 \times 10^{-5} \mathrm{rad}\right)$ at a wide range of speeds. Several acrylic stands - for different experiments - were made in-house to hold an object at an optimum height above the rotary table, where the projection of the object would fall in the central area of the FPD (Figure 3.1).

\subsubsection{Collimation}

The x-ray tube was enclosed in a wooden box with a protective lead sheet that also acted as a collimator for our experiment. While the collimator was set to block the unwanted portions of the x-ray beam, it would have been difficult to design an adjustable collimator to dynamically filter out the unwanted portion of the x-ray beam based on the system geometric parameters, such as the source to detector distance and the tilting angle of the FPD. Therefore, the collimation was not precisely adjusted to focus the beam exactly on the active area of the FPD. Nevertheless it would partially block the unwanted x-ray beams that otherwise might have added to the noise level of the FPD projection images by scattering off the objects in the vicinity of the VRX-CBCT test bed.

\subsubsection{Terminology}

Before explaining the details of the VRX-CBCT setup, it is important to define some of the common terms used in this document. Figure 3.2 illustrates a conventional CBCT system. The rotary table is located between the x-ray tube and the FPD, which 


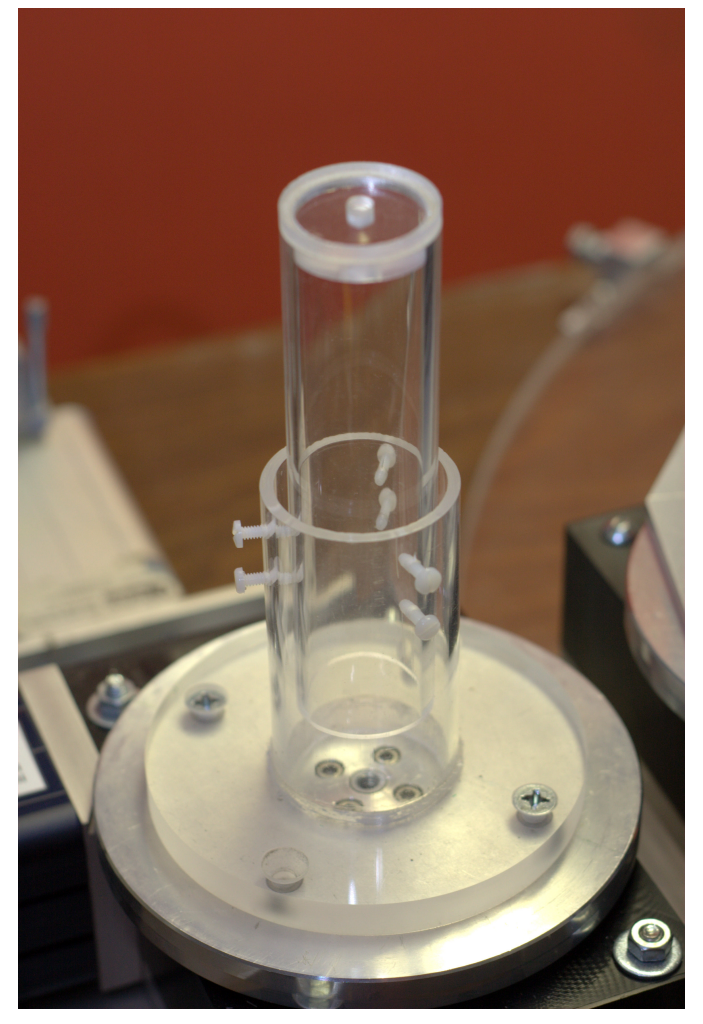

Figure 3.1: Adjustable acrylic stand for the rotary table.

A $15 \mathrm{~cm}$ long acrylic tube was placed inside another acrylic tube of height $7.5 \mathrm{~cm}$. Six nylon screws on the side held the first cylinder in place while allowing for adjustment of undesired lateral movement of the stand. 


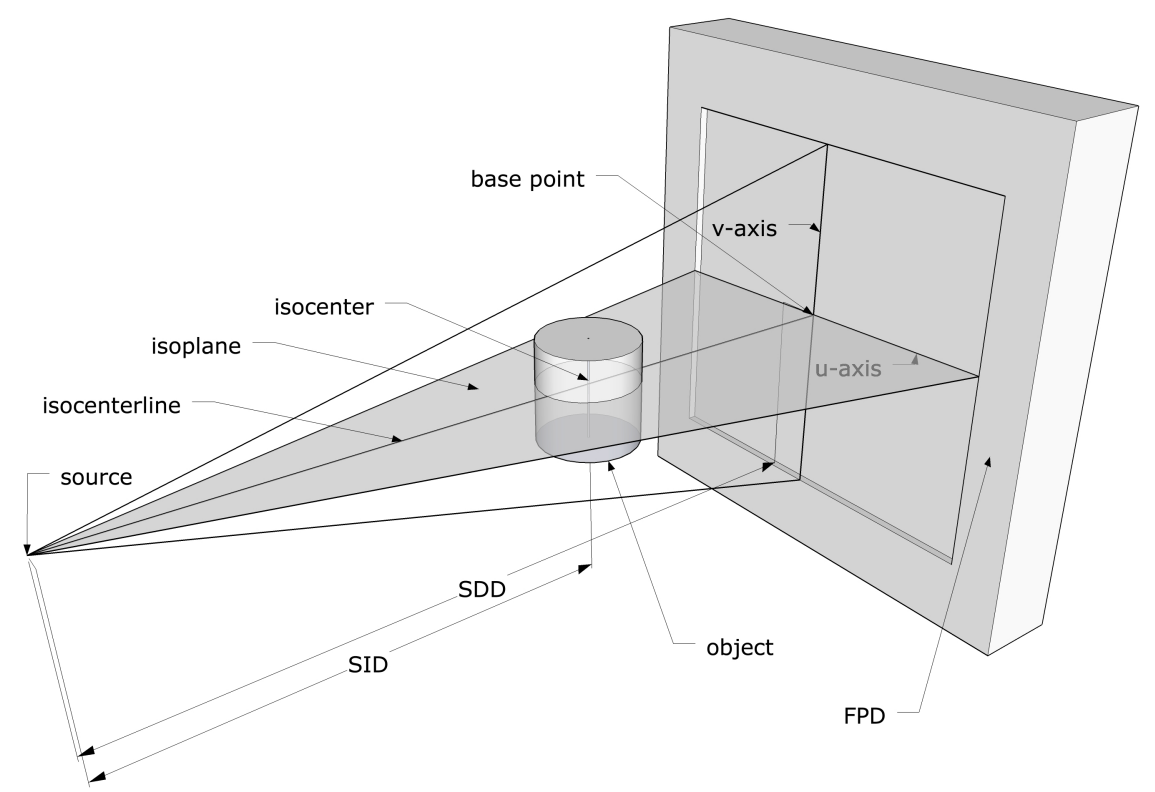

Figure 3.2: Illustration of a conventional CBCT.

The object is placed between the x-ray source and the FPD. In clinical CBCTs, the source and the FPD rotate about a stationary patient. 
are both stationary. The center of the focal spot in the x-ray tube is called the $x$-ray source or source in short. The axis normal to the surface of the rotary table that passes through the center of rotation (COR) of the rotary table is called the axis of rotation. Horizontal isoplane, or isoplane in short, is the unique horizontal plane that passes through the x-ray source. This plane intersects the axis of rotation at a point called the isocenter.

The isocenter is the origin of the object coordinate system. The z-axis falls on the axis of rotation pointing up, the $x$-axis points toward the source, and the $y$-axis is $\vec{z} \times \vec{x}$. Intuitively, the $x$ - and $y$-axes fall on the isoplane. The unique vertical plane that is formed by the $x$ - and $z$-axes of the object coordinate system is the vertical isoplane. The two isoplanes should split the x-ray cone beam into four symmetric quarter-cones. The line formed by the intersection of the two isoplanes, which passes through both the source and the isocenter, is called the isocenterline. The x-ray beam that falls on the isocenterline is called the central $x$-ray beam. Since in our experiments the latter two entities have the same geometry, they may be used interchangeably throughout this document.

The center of the imaging surface is called the FPD center point, and is the origin of the FPD coordinate system or the uvw system. The u-axis runs along the FPD data matrix rows, and the $v$-axis runs along its columns, with the top-right quadrant being the first (i.e., positive-positive) quadrant. The w-axis is defined as $\vec{u} \times \vec{v}$. The line that connects the source to the center of the imaging surface on the FPD is called the FPD centerline. The isocenterline intersects the FPD's imaging surface, or the extension of that surface, at the base point. The angle between the isocenterline and the normal vector $\vec{n}$ of the imaging surface plane is called vr $x_{v}$ when the FPD is tilted exclusively about its $v$-axis. Using similar definition, $v r x_{u}$ is the angle that $\vec{n}$ forms with the isocenterline when the FPD is tilted exclusively about its $u$-axis.

It is important to emphasize that although ideally in our experiment the FPD centerline should match the isocenterline, in most cases these two lines are not the same. In our settings, the location $(u, v)$ of the base point in the FPD $u v$ coordinate system is called base point offset, or offset in short. The intersection of the horizontal and vertical isoplanes and the imaging surface of the FPD are called the horizontal and vertical FPD midlines respectively. In theory, the horizontal FPD midline stays in the same location when the $v r x_{v}$ and $v r x_{u}$ angles change, therefore the FPD detector row that falls on the midline is a unique detector row, called the FPD midrow, which can act as a linear array detector to reconstruct a single CT image independent of the vrx angles. The plane that such a 2D CT image falls on is called the CT midplane in our experiments, and is used to calculate the transverse spatial resolution of the system. 


\subsection{Transverse Spatial Resolution Analysis}

Figure 3.3 shows the first generation VRX-CBCT test bed. The first generation of the VRX-CBCT was developed to mainly focus on comparing the VRX effect in CT images acquired using the FPD [64] to the results from the conventional VRX-CT with a linear detector array [30]. Therefore the FPD frame was designed to only allow tilting about the $z^{\prime}$ vertical axis parallel to the $z$-axis of the object coordinate system. In this part of the experiment, any FPD rotations about other axes orthogonal to the $z^{\prime}$-axis were insignificant by design and were assumed to be zero for simplicity.

\subsubsection{Experiment Setup}

\subsubsection{Flat Panel Detector}

The FPD was mounted inside an aluminum enclosure made in-house with the help of the Biomedical Instrumentation Division at the University of Tennessee Health Science Center. A horizontal handle on top of the box controlled a rigid rod connected to one side of a frame that holds the FPD inside the box, which enabled us to rotate the FPD about one of its vertical sides. The center of the rod was $13 \mathrm{~mm}$ from the edge of the FPD. Using the handle on top, the FPD could rotate about the rod called the $z^{\prime}$-axis herein - from the default position at $90^{\circ}$, where it was perpendicular to the central x-ray beam, to $0^{\circ}$, where the imaging plate was parallel to the central x-ray beam. Although the FPD rotated about the $z^{\prime}$-axis, to be consistent throughout this document we have used the term $v r x_{v}$ to refer to the tilt angle of the FPD in this manner.

It is imperative to mention that in this generation of the VRX-CBCT, since the FPD rotates about the $z^{\prime}$-axis rather than the $v$-axis, the isocenterline does not fall on the FPD centerline at $v r x_{v}$ angles other than $90^{\circ}$. As the $v r x_{v}$ angle decreases, the isocenterline diverges away from the $z^{\prime}$-axis, leaving the base point at a farther distance from the FPD center point as well as the source. Therefore at each $v r x_{v}$ angle in the first generation VRX-CBCT, the position of the FPD enclosure box along the $y$-axis - relative to the position of the source and the isocenter - was adjusted for the isocenterline and the FPD centerline to match. If FPD position were left unadjusted, the actual $v r x_{v}$ angle would be greater than the tilt angle of the FPD. In addition, the x-ray photon distribution on the FPD would be less homogenous due to the more severe $\mathrm{x}$-ray exposure asymmetry away from the isocenterline. The alignment of isocenterline to match the FPD centerline in the first generation VRX-CBCT was achieved by moving both the x-ray tube and the rotary table proportionally in the same direction along the $y$-axis using the precision linear moving tables. 


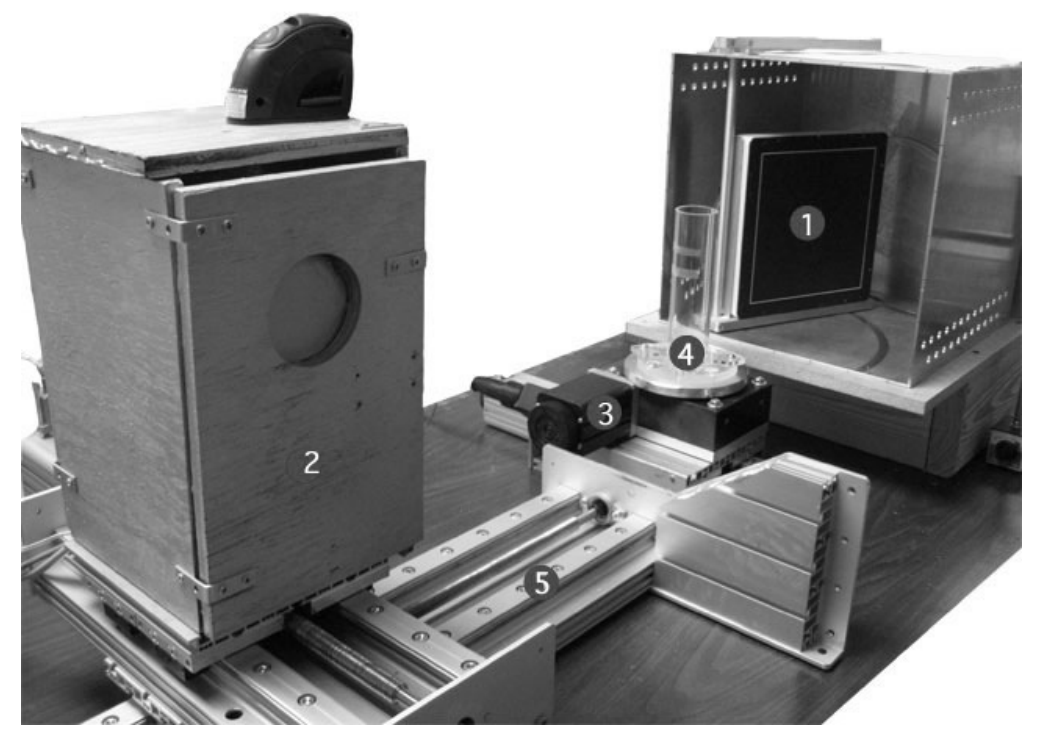

Figure 3.3: First generation VRX-CBCT test bed.

The system consists of (1) a flat panel detector, (2) a micro-focal x-ray tube, (3) a rotary table, (4) an MTF phantom on an acrylic stand, and (5) the moving mechanism for the x-ray tube and the rotary table. 


\subsubsection{MTF Phantom}

An MTF phantom was designed in-house to measure the transverse spatial resolution of the VRX-CBCT (Figure 3.4). The phantom consisted of a thin layer of copper foil with the thickness of $76 \mu \mathrm{m}\left(0.003^{\prime \prime}\right)$ sandwiched between two halfcylinders made of ultra high weight polyethylene (UHP). The cylinder was $22 \mathrm{~mm}$ tall and $32 \mathrm{~mm}$ in diameter. The MTF phantom was placed on a custom-made stand with one of its flat surfaces facing down and the copper foil aligned vertically. The stand was then attached to the rotary table using bolts, and the phantom was fixed in place to prevent any undesired movement during the $\mathrm{CT}$ data acquisition process.

\subsubsection{System Parameters}

Table 3.3 describes the geometric and operating parameters of the first generation VRX-CBCT. The rotary table was placed as close as possible to the FPD to minimize the magnification effect. The source to isocenter distance (SID) was fixed at $72.8 \mathrm{~cm}$, with the source to detector distance (SDD) variable between $80.9 \mathrm{~cm}$ at $v r x_{v}=90^{\circ}$ to $93.8 \mathrm{~cm}$ at $v r x_{v}=30^{\circ}$. This distance allowed for an acceptable minimum level of projection image (PI) signal-to-noise ratio (SNR) of $14.5 \mathrm{db}$.

Although the maximum voltage of the x-ray tube is $80 \mathrm{kVp}$, our initial experiments showed increasing noise at voltage levels in the vicinity of the maximum value. To reduce this effect, the x-ray tube voltage was set to $75 \mathrm{kVp}$ throughout this experiment. The x-ray tube current was initially set at $150 \mu \mathrm{A}$ for $v r x_{v}=90^{\circ}$ and adjusted up to $250 \mu \mathrm{A}$ at smaller angles to maintain an acceptable SNR.

\subsubsection{FPD Pre-processing and Data Acquisition}

The gain calibration, offset calibration, and acquisition of the projection data were performed using $\mathrm{ViVA}^{\mathrm{TM}}$ software provided by the manufacturer of the FPD. To capture projection images, the rotation speed of the rotary table was set to $2.97^{\circ}$ per second $(0.052 \mathrm{rad} / \mathrm{s})$ and the FPD acquired the projection images at the rate of 15 fps. The total scan time was 121 s per sinogram, which resulted in 1818 views for a full $360^{\circ}$ scan. The projection data for each experiment were then processed to form a $3 \mathrm{D}$ sinogram.

\subsubsection{Calibration}

To reconstruct the projection data into CT images with minimal artifacts, it is essential to find the exact values of some of the geometric parameters that contribute significantly to the final CT image. These parameters are SID, SDD, vr $x_{v}$, and base point horizontal and vertical offsets. 


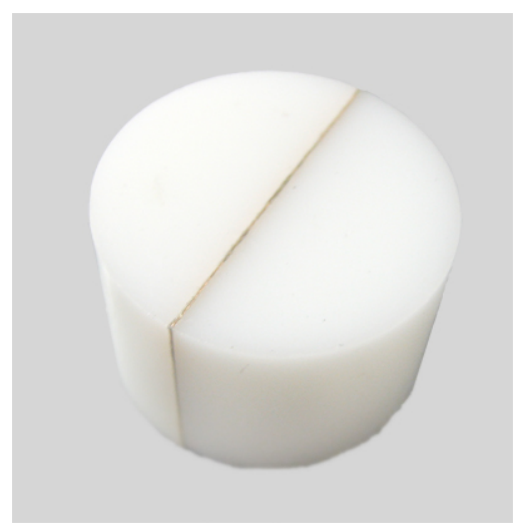

Figure 3.4: Transverse MTF phantom.

A $76 \mu \mathrm{m}$ copper foil is sandwiched between two half-cylinders of UHP.

Table 3.3: VRX-CBCT parameters for transverse resolution analysis.

\begin{tabular}{llll}
\hline Parameter & Value & Parameter & Value \\
\hline resolution & $512 \times 512(2 \times 2$ binning $)$ & tube voltage & $75 \mathrm{kVp}$ \\
pixel pitch & $384 \mu \mathrm{m}$ & tube current & $150 \mu \mathrm{A} @ 90^{\circ}$ \\
SID & $72.8 \mathrm{~cm}$ & & $250 \mu \mathrm{A} @ 45^{\circ}$ \\
SDD & $80.9 \mathrm{~cm} @ 90^{\circ}$ & $250 \mu \mathrm{A} @ 30^{\circ}$ \\
& $91.4 \mathrm{~cm} @ 45^{\circ}$ & FPD frame rate & $15 \mathrm{fps}$ \\
& $93.8 \mathrm{~cm} @ 30^{\circ}$ & rotary table speed & $0.052 \mathrm{rad} / \mathrm{s}$ \\
\hline
\end{tabular}


A modified version of the original VRX-CT calibration algorithm $[67,60]$ was used in this phase of our study. The algorithm was written in Matlab ${ }^{\circledR}$ (The Mathworks Inc., USA). Figure 3.5 shows the custom-made calibration phantom used in this experiment. A $0.4 \mathrm{~mm}$ thick lead wire was mounted on the side of a $22.5 \mathrm{~cm}$ acrylic tube of external diameter $5.1 \mathrm{~cm}$. The phantom was then mounted on the rotary table and aligned so that the central axis of the tube was aligned with the axis of rotation. The $2 \mathrm{D}$ projection images of the phantom were obtained for a full $360^{\circ}$ view at different vr $_{v}$ angles.

To calibrate using the modified 2D VRX calibration, it is important to use the sinogram created from the horizontal FPD midline. As a reminder, the FPD midline by definition does not move on the FPD when the $v r x_{v}$ angle changes, keeping the same slope and $v$-intercept value on the FPD $u v$ coordinate system. To find the midline, a set of 3 steel rods, connected together to form a sheet of rods $2.54 \mathrm{~cm}\left(1^{\prime \prime}\right)$ apart was held between the source and the FPD perpendicular to the isocenterline. All rods were then aligned to be horizontal using an inclinometer with a precision of $0.5^{\circ}$. Three x-ray projection images of the grid were taken at $v r x_{v}$ angles of $65^{\circ}, 45^{\circ}$, and $30^{\circ}$. At each angle, the $2 \mathrm{D}$ projection images of the parallel rods were analyzed to extract three non-parallel lines $L_{1}, L_{2}$, and $L_{3}$ :

$$
L_{n}: \frac{y_{n}-y_{0}}{x_{n}-x_{0}}=\frac{y_{n}^{\prime}-y_{n}}{x_{n}^{\prime}-x_{n}}=m_{n},
$$

where $n=1,2,3$. The slope of the FPD midline $m_{\text {mid }}$ should be zero by definition, therefore the equation for the midline is reduced to $y_{\text {mid }}=y_{0}$. If $m_{n}=0$ for the $n$th line, that line would be the exact solution for the FPD midline. Otherwise by choosing two adjacent lines $L_{i}$ and $L_{i+1}$ so that $m_{i} \times m_{i+1}<0$ :

$$
\frac{y_{i}-y_{0}}{x_{i}-x_{0}} \times m_{i+1}=\frac{y_{i+1}-y_{0}}{x_{i+1}-x_{0}} \times m_{i} .
$$

But $x_{i}=x_{i+1}$ since they are on the same column of the FPD, therefore:

$$
\begin{gathered}
\left(y_{i}-y_{0}\right) \times m_{i+1}=\left(y_{i+1}-y_{0}\right) \times m_{i} \\
y_{m i d}=y_{0}=\frac{y_{i} \cdot m_{i+1}-y_{i+1} \cdot m_{i}}{m_{i+1}-m_{i}} .
\end{gathered}
$$

The closest FPD detector row to the midline was then selected as the FPD midrow.

To create a $2 \mathrm{D}$ sinogram to be used by the calibration algorithm, the intensity values of all pixels of the FPD midrow were used (Figure 3.6). The sinogram was then analyzed using a simple thresholding technique and the center of intensity was calculated for each view to extract the location of the pin.

The calibration algorithm uses the estimated values for the geometric parameters of the system to simulate a $2 \mathrm{D}$ pin sinogram using the following formula:

$$
u=S D D \times \frac{\sin \beta}{\sin \left(v r x_{v}-\beta\right)},
$$




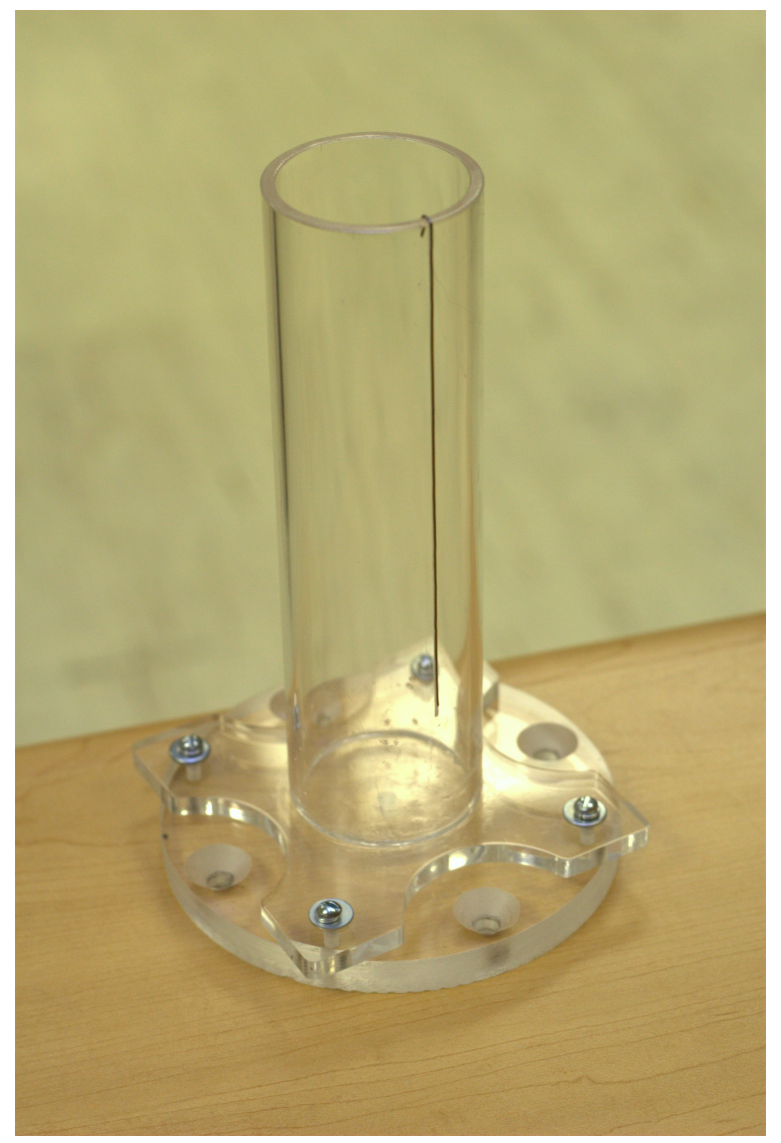

Figure 3.5: Calibration phantom for the first generation VRX-CBCT. The phantom consists of a thin lead wire mounted on the side of an acrylic tube. 


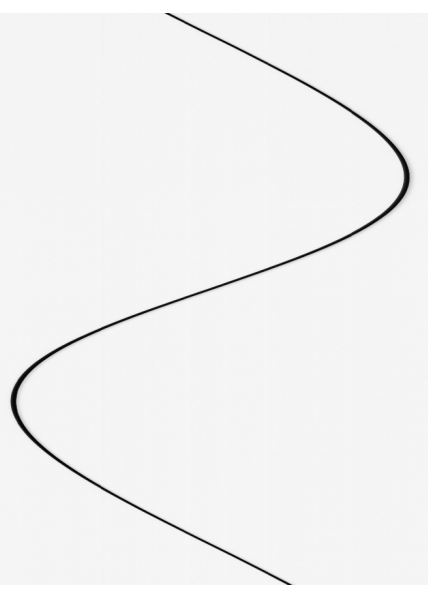

Figure 3.6: Example of a pin sinogram at the midplane.

Each row of the sinogram contains the intensity values of the projection image on the midrow at a specific pin phase $\theta$. In other words, the vertical axis in the sinogram corresponds to $\theta$ and the horizontal axis corresponds to $p$, the pin's x-ray projection profile on the midrow of the FPD. The pin phase changes from $\theta_{0}$ for the top row, to $\theta_{0}+2 \pi+\epsilon$ for the bottom row $(\epsilon>0)$. 
where the fan angle $\beta$ is dependent on the $\operatorname{SID}$, pin radius $r$, and phase $\theta$, and can be calculated using the formula below:

$$
\beta=\arctan \left(\frac{r \cdot \sin \theta}{r \cdot \cos \theta+S I D}\right)
$$

For each view, the sum of square error (SSE) between the location of simulated data and the actual pin was calculated and then divided by the number of views to form the normalized sum of square error of the simulated data. The algorithm uses an unconstrained nonlinear optimization [67] method to minimize the normalized SSE value. The iterative minimization algorithm stops when the difference between the answers in two consecutive iterations is less than $10^{-7}$, or when the number of iterations exceeds 2000. The SID, SDD, vr $x_{v}$, and the base point horizontal offset value obtained from the minimization algorithm were then stored to be used in the reconstruction algorithm.

\subsubsection{CT Reconstruction and MTF Calculation}

\subsubsection{CT Reconstruction Algorithm}

An algorithm similar to the original Feldkamp algorithm (FDK) [45] was developed to reconstruct a 3D CT image. Although the basic concepts of both methods are the same, the FDK method could not be directly implemented in our VRX-CBCT system. In a conventional CBCT, the projection of any single layer of CT image on the imaging surface is assumed to be a horizontal line that falls on a single row of the FPD detector matrix. In VRX-CBCT however, as we move further away from the CT midplane, such projection data are less likely to fall exactly on any single FPD row. Instead, the intensity values on the FPD that are used to reconstruct a single layer of CT other than the midplane are located on a line with a non-zero slope in the FPD $u v$ coordinate system. Therefore the absolute value of the slope for any given non-midplane CT layer increases as the $v r x_{v}$ angle decreases.

To correct for this effect, the intensity values of the FPD projection data for each view were first re-mapped to a new set of intensity values on a virtual FPD detector so that each row of the virtual FPD would contain the intensity values required to reconstruct one $\mathrm{CT}$ image using the FDK method. To form the virtual FPD intensity matrix, first the values of the last column on the far side of the actual FPD were placed directly into the last column of the virtual detector. The expression far side is used for the vertical or horizontal side of the actual FPD that sits farther away from the source, when the FPD rotates about the $v$-axis or the $u$-axis respectively. The values of the remaining columns of the virtual FPD intensity matrix were then calculated using the interpolation method.

Another difference between our algorithm and FDK is that while in the latter method the weighted 2D sinograms for each CT layer are directly used for fan beam reconstruction, in our case the sinograms were first re-mapped to form virtual parallel 
sinograms using a fan beam to parallel beam algorithm. The new intensity matrix was then filtered using Shepp-Logan filter [68] and backprojected using parallel beam inverse Radon transformation [69].

In addition, our FPD projection data have been already pre-processed for signal homogeneity during the FPD gain calibration process, so the FDK weighting matrix was not included in our method.

As with the calibration program, the CT reconstruction program was written in Matlab ${ }^{\circledR}$ programming environment, with the filtered backprojection section implemented in standard $\mathrm{C}$ for faster performance.

\subsubsection{MTF Calculations}

Four $v r x_{v}$ angles of $90^{\circ}, 65^{\circ}, 45^{\circ}$, and $30^{\circ}$ were chosen for this experiment. For each angle, two sets of calibration data and five projections of the MTF phantom were acquired. For each experiment at a specific $v r x_{v}$ angle, the phantom was placed on a platform on top of the rotary table in a random location and in a random orientation within the field of view (FOV) of the detector. This randomization reduces the effect of non-isotropic CT reconstruction in case such phenomenon is present in the final image. The CT images of the phantom were then reconstructed using the algorithm described in section 3.3.4.1 and two adjacent slices near the isoplane were chosen.

The 20 resulting CT images of the phantom ( 5 experiments per angle $\times 2$ calibrations per experiment $\times 2$ adjacent $\mathrm{CT}$ slices) were then rotated to make the foil vertical. All the rows in a region of interest (ROI), chosen to be a square inscribed in the outline of the MTF phantom, were averaged to form the line spread function (LSF). The LSF curve was then adjusted for the DC value, which was set to be the minimum value of the LSF function plus $1 \%$ of the peak value. The Fourier transform of the LSF was then calculated to form the optical transfer function (OTF). The absolute value of OTF was normalized to its value at zero frequency to form the MTF at each $v r x_{v}$ angle. The resulting $20 \mathrm{MTF}$ functions were averaged to reduce the influence of noise in the final MTF for each specific angle.

\subsection{Axial Spatial Resolution Analysis}

In the second phase of the study, we studied the effect of changes in $v r x_{u}$ angle on the spatial resolution of the 3D CT images along the $z$-axis of the object coordinate system, also known as the CT axial direction [65]. In order to examine such effect, the VRX-CBCT system was redesigned to allow the FPD to tilt about its $u$-axis.

Figure 3.7 shows the second generation VRX-CBCT test bed. There were a few major changes made to the FPD movement mechanism compared to the first generation VRX-CBCT. The most important improvement was the implementation 


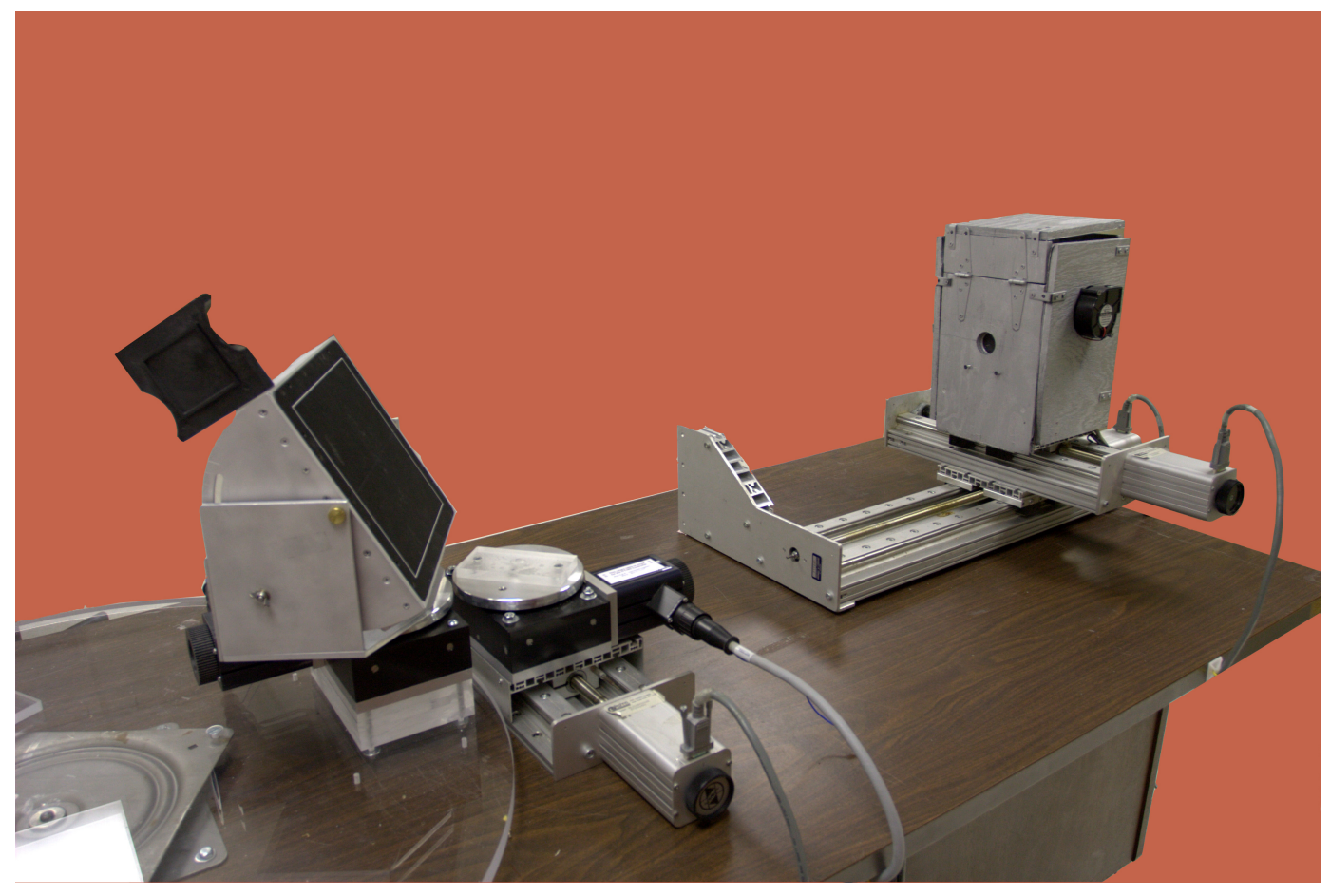

Figure 3.7: Second generation of the VRX-CBCT test bed.

A redesigned detector frame allows FPD rotations about both $v$ - and $u$-axis. 
of a new FPD aluminum frame, designed and built in-house, which allowed tilting the detector about $u$-axes (Figure 3.8). In addition, the frame was installed on a second rotary table, allowing for precise independent changes in the $v r x_{v}$ angle. The rotating axis of the frame and the FPD rotary table were designed to approximately match the $u$ - and $v$-axes of the FPD, respectively. This new feature is designed to keep the separation of the isocenterline and the FPD centerline at a minimum level, when the VRX-CBCT is initially calibrated correctly. As a reminder, in the first generation of the system the FPD could rotate only about the $z^{\prime}$-axis, and not the $v$-axis, which called for realignment of the source and the isocenter every time the $v r x_{v}$ angle changed. Because of the way the new frame was designed, in this part of the experiment any FPD rotation about its $w$-axis was insignificant, and was assumed to be zero for simplicity.

\subsubsection{Experiment Setup}

\subsubsection{Flat Panel Detector}

In the second phase of our experiment, the FPD rotary table was moved to the position where the FPD and its frame were perpendicular to the isocenterline. A geometrical method using laser beam and reflection off the FPD surface was used to find the exact rotation angle of the FPD rotary table that forms a vr $x_{v}$ angle of $90^{\circ}$ (Figure 3.9). The angular error of the laser calibration was measured to be less than $0.01^{\circ}$, however the overall accuracy of our measurement may have been limited by other factors such as the manufacturing error in the FPD frame and the smoothness of the carbon fiber cover of the FPD. Nevertheless, the calibration program attempts to correct small errors in the $v r x_{v}$ angle measurements before the projection images are reconstructed into $\mathrm{CT}$ images.

To measure the effect of changing the $v r x_{u}$ angle on the axial spatial resolution of the CT images, the FPD was titled about its $u$-axis to form vr $x_{u}$ angles of $90^{\circ}$, $30^{\circ}$, and $20^{\circ}$ while the $v r x_{v}$ angle was fixed at $90^{\circ}$. A magnetic inclinometer with the precision of $0.5^{\circ}$ was attached to the top part of the FPD frame to display the best estimate for the $v r x_{u}$ angle. The frame was then fixed in place after the desired tilting angle was obtained.

\subsubsection{MTF Phantom}

A second customized MTF phantom was designed and manufactured to measure the axial spatial resolution of the VRX-CBCT (Figure 3.10). The axial MTF phantom consists of a $4 \mathrm{~mm} \times 10 \mathrm{~mm}$ layer of copper foil with the thickness of $25 \mu \mathrm{m}\left(0.001^{\prime \prime}\right)$, sandwiched between two cubes of acrylic $2.54 \mathrm{~cm}\left(1^{\prime \prime}\right)$ per side. The MTF phantom was placed on top of the rotary table stand described earlier so that the copper foil would be horizontal. As with the previous setup, the phantom was fixed in place to 


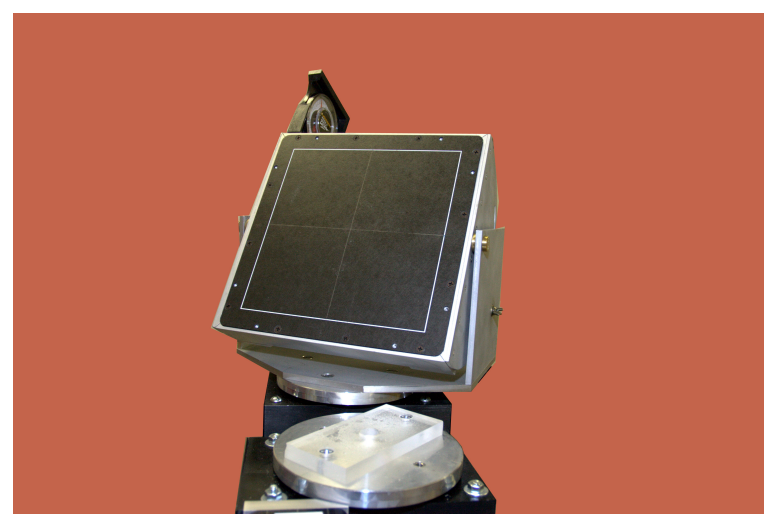

(a)

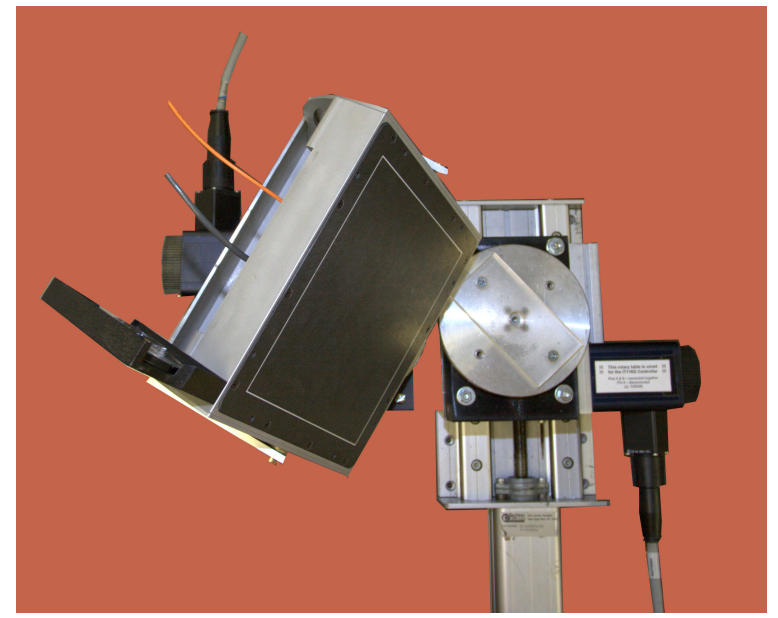

(b)

Figure 3.8: FPD rotation system in the second generation VRX-CBCT. (a) Front view along the $x$-axis, and (b) top view along the $z$-axis of the object coordinate system.

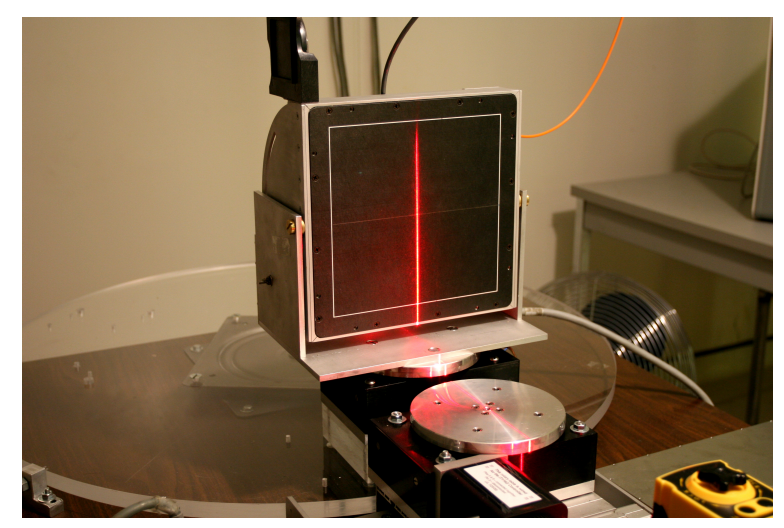

Figure 3.9: VRX-CBCT alignment.

The the laser line passes through the isocenter, reflects from the FPD surface at the vertical midline and falls on the source after passing again through the isocenter. 


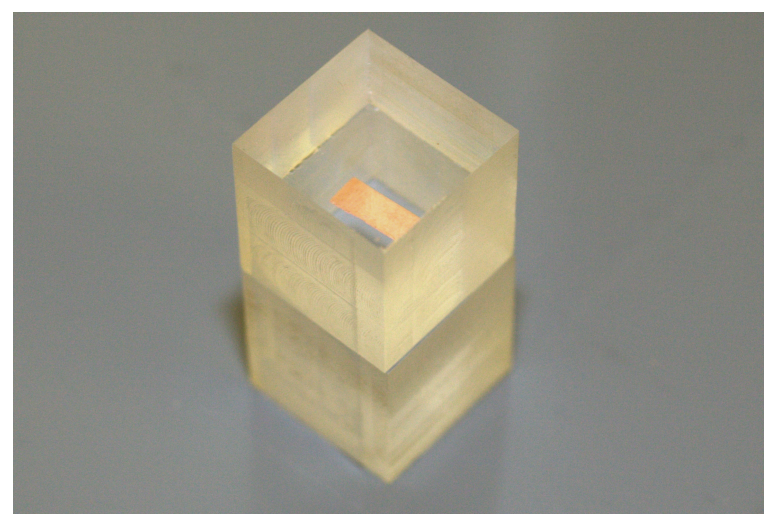

Figure 3.10: Axial MTF phantom.

A $25 \mu \mathrm{m}$ thick copper foil is placed between two acrylic cubes.

prevent any lateral movement during the $\mathrm{CT}$ data acquisition process. The stand was aligned to be vertical using six plastic screws on the side of the stand base with maximum lateral movement error of $25 \mu \mathrm{m}\left(0.001^{\prime \prime}\right)$.

\subsubsection{System Parameters}

Table 3.4 describes the geometric and operating parameters of the second generation VRX-CBCT. The isocenter to detector distance was slightly increased to $16.5 \mathrm{~cm}$ compared to the first generation VRX-CBCT to allow the FPD to rotate freely about its $u$-axis without interfering with the rotary table stand. To increase the homogeneity of the x-ray beam and achieve a similar magnification effect compared to the first experiment, the source to isocenter distance (SID) was increased to $102 \mathrm{~cm}$.

The tube voltage was fixed at $74 \mathrm{kVp}$ for the entire experiment. Both the tube current and the FPD frame rate were variable from $150 \mu \mathrm{A}$ and $15 \mathrm{fps}$ for $v r x_{u}=90^{\circ}$ to $250 \mu \mathrm{A}$ and $5 \mathrm{fps}$ at vr $x_{u}=20^{\circ}$ to maintain an acceptable SNR within the linear range of FPD.

\subsubsection{FPD Pre-processing and Data Acquisition}

As with the previous experiment, the gain calibration, offset calibration, and acquisition of the projection data were done using ViVA ${ }^{T M}$ application. Two different rotation speeds were used to synchronize the rotary table with the acquisition rate of the FPD. For FPD frame rate of $15 \mathrm{fps}$, the rotation speed of the CT rotary table was set to $2.97^{\circ}$ per second $(0.052 \mathrm{rad} / \mathrm{s})$. This speed was reduced to $0.99^{\circ}$ per second $(0.017 \mathrm{rad} / \mathrm{sec})$, where we were forced to use the smaller FPD frame rate of $5 \mathrm{fps}$ to enhance the SNR for $v r x_{u}$ of $30^{\circ}$ and $20^{\circ}$. The total scan time was $121.2 \mathrm{~s}$ per sinogram for $\operatorname{vr} x_{u}=90^{\circ}$, and $363.6 \mathrm{~s}$ for the latter two angles. Out of 2048 projection 
Table 3.4: VRX-CBCT parameters for axial resolution analysis.

\begin{tabular}{llll}
\hline Parameter & Value & Parameter & Value \\
\hline resolution & $512 \times 512(2 \times 2$ binning $)$ & tube voltage & $74 \mathrm{kVp}$ \\
pixel pitch & $384 \mu \mathrm{m}$ & tube current & $150 \mu \mathrm{A} @ 90^{\circ}$ \\
SID & $85.5 \mathrm{~cm}$ & & $250 \mu \mathrm{A} @ 30^{\circ}$ \\
SDD & $102.0 \mathrm{~cm}$ & $250 \mu \mathrm{A} @ 20^{\circ}$ \\
& & FPD frame rate & $15 \mathrm{fps} @ 90^{\circ}$ \\
& & & $5 \mathrm{fps} @ 30^{\circ}$ \\
& & & $5 \mathrm{fps} @ 20^{\circ}$ \\
& & rotary table speed & $0.052 \mathrm{rad} / \mathrm{s} @ 90^{\circ}$ \\
& & & $0.017 \mathrm{rad} / \mathrm{s} @ 30^{\circ}$ \\
& & $0.017 \mathrm{rad} / \mathrm{s} @ 20^{\circ}$ \\
\hline
\end{tabular}

images obtained, 1818 views (representing a full $360^{\circ}$ scan) were selected to form a $3 \mathrm{D}$ sinogram.

\subsubsection{Calibration}

The calibration process described in section 3.3.3 was used to find the exact value of the system geometric parameters SID, SDD, vr $x_{v}$, and horizontal base point offset. To correctly reconstruct the CT images, it was essential to find two other important parameters of the system: vr $x_{u}$ angle and FPD horizontal midline.

Before discussing the calibration of $v r x_{u}$, two important facts must be addressed. When the vrx $x_{v}$ angle is fixed at $90^{\circ}$ and $v r x_{u}$ angle is changed, the projection of each layer of the object being scanned falls approximately on a line parallel to the rows of the FPD. Another fact is that although finding the exact value of $v r x_{u}$ is important, this value is eventually used to address the horizontal distortion of the object being scanned along different rows of the detector due to the different magnification levels. In other words, the projection of two fixed points on the object being scanned that are not lying on the vertical isocenterline are closer on the near side, and farther away on the far side of the FPD compared to their distance on the FPD midline. Therefore an alternative to finding the exact value of $v r x_{u}$ is to bypass this process and directly remap the intensity values on the FPD using a similar technique described in the previous experiment.

The initial calibration to find SID, SDD, vr $x_{v}$, and base point offset was done using the lead wire on acrylic tube phantom described earlier. However to find the FPD horizontal midline, another phantom was manufactured using a smaller acrylic tube of $25.4 \mathrm{~mm}$ diameter, and three balls with an approximate diameter of $100 \mu \mathrm{m}$ each, placed along the side of the acrylic tube at equal distances of $25.4 \mathrm{~mm}\left(1^{\prime \prime}\right)$ from one another. The phantom was then rotated to position the balls at a phase angle of $-\frac{\pi}{2}$ (i.e., where the $y$ components of the balls were at their maximum value). 
First, two snapshots of the phantom were taken at $v r x_{u}$ angle of $90^{\circ}$ at rotary table phase difference of $\delta \theta=\theta_{2}-\theta_{1}=\pi$. The locations of the projection of the three lead balls in the first snapshot were $\left(u_{1}, v_{1}\right),\left(u_{2}, v_{2}\right)$, and $\left(u_{3}, v_{3}\right)$ and in the second snapshot were $\left(u_{1}^{\prime}, v_{1}\right),\left(u_{2}^{\prime}, v_{2}\right)$, and $\left(u_{3}^{\prime}, v_{3}\right)$. The distances $d_{i}=u_{i}^{\prime}-u_{i},(i=1 \ldots 3)$ were calculated at $\operatorname{vr} x_{u}=90^{\circ}$ along with the average distance $\bar{d}=\frac{1}{3} \sum_{i=1}^{3} d_{i}$. For the $v r x_{u}$ angle of $20^{\circ}$, the distances $d_{i}^{\prime}$ between the two views of the balls varied due to magnification differences between the far side and the near side. Using interpolation technique, $v_{0}$ was selected so that $d^{\prime}\left(v_{0}\right)=\bar{d}$. The row that was the closest to $v=v_{0}$ was the FPD midrow, and $v_{0}$ was set to be the vertical offset.

\subsubsection{CT Reconstruction and MTF Calculation}

\subsubsection{CT Reconstruction Algorithm}

Similar to the technique used in section 3.3.4.1, the intensity values of the FPD projection data for each view were re-mapped to a new set of intensity values on a virtual FPD detector. The purpose of this conversion was to correct for the variable magnification effect caused by tilting the FPD. To form the virtual FPD intensity matrix, the values of the last row on the far side of the FPD were put directly into the last column of the virtual detector, and the remaining rows of the virtual FPD intensity matrix were calculated by interpolating between the actual intensity values on the real FPD.

For each row in the ROI of the virtual FPD intensity matrix, the intensity values of that row for each view were compiled to form a single 2D sinogram. The algorithm previously described in section 3.3.4.1 was then used to reconstruct a stack of $\mathrm{CT}$ images from the collection of the 2D sinograms.

\subsubsection{MTF Calculations}

The MTF phantom described in section 3.4.1.2 was placed on the rotary table stand within the FOV. The stand height was adjusted so that the center of the MTF phantom would be approximately on the isoplane, where the projection of the midplane falls on the FPD midline. The calculated MTF using this method was anticipated to be the maximum practical MTF of the VRX-CBCT system at each vr $x_{u}$ angle.

For each $\operatorname{vr} x_{u} \in\left\{90^{\circ}, 30^{\circ}, 20^{\circ}\right\}$, three sets of projection images of the MTF phantom were acquired. For each set, however, the location of the MTF phantom on the stand was randomly chosen within the FOV. Such random placement of the MTF phantom was expected to help achieve a more realistic MTF measurement by reducing the effect of geometrical MTF variations.

A stack of 3D CT images was obtained from the series of $2 \mathrm{D}$ sinograms. For the MTF measurement in this experiment, the 3D CT image was viewed as a stack 
of sagittal slices rather than a series of cross-sectional CT slices. The sagittal images of the MTF phantom were rotated so that the phantom's copper foil was aligned horizontally.

The intensity values of each column that crossed the thin foil in the sagittal slices formed a single LSF. An ROI consisting of 15 such columns was selected. Also for this experiment, the sagittal slice that fell on the vertical isoplane and its two adjacent slices were chosen to be included in the LSF measurement. This procedure resulted in a total of $135 \mathrm{LSF}$ curves per $v r x_{u}$ angle, where there were 3 independent samples (one for each PI acquisition) and $3 \times 15$ dependent LSFs ( 3 adjacent sagittal slices $\times 15$ columns in the ROI per sagittal slice).

The LSF curves were adjusted for the DC value, which was set to be the minimum value of the LSF function. The lower $1 \%$ of the LSF was cut and replaced with zeros to reduce the effect of high frequency noise. The Optical Transfer Function (OTF) of each modified LSF was then calculated using the Fast Fourier Transform (FFT) method. All the OTFs were averaged to form a single OTF for each vr $x_{u}$ angle. The MTF curve was then calculated for each angle by dividing the absolute value of the OTF function by its value at zero frequency.

\subsection{Isotropic Spatial Resolution Analysis}

In the third phase of the study, we analyzed the effect of changes in the transverse and axial spatial resolutions of the VRX-CBCT, when the vr $x_{v}$ and vr $x_{u}$ angles changed simultaneously. One of the objectives in this experiment was to minimize any simplification assumptions about the projection data, and eliminate the need to precondition the projection images. In other words, in the third and final phase of the study, we abandoned our previous practice of using a simple FDK-based algorithm and instead developed a completely new approach to reconstruct the 3D CT images from the 2D projection images acquired using the VRX-CBCT system.

\subsubsection{Experiment Setup}

\subsubsection{Flat Panel Detector}

We use the notation $v r x_{v u}$ for the isotropic angles formed when the FPD is rotated about its $v$-axis to an angle measured as $v r x_{v u}$, followed by a rotation about the $u$-axis for the same value. As an example, the $v r x_{v u}=45^{\circ}$ implies that the FPD is first rotated $45^{\circ}$ about its $v$-axis and then $45^{\circ}$ about its $u$-axis. Since $v r x_{u}$ and $v r x_{v}$ are the angles between the isocenterline and its $u$ and $v$ components respectively, $v r x_{v u}$ is not the same as the actual angle between the FPD imaging surface and the 
isocenterline $(\tau)$, which can be calculated using the following formula:

$$
\tau=\frac{v r x_{v u}}{\sqrt{2}}
$$

Nevertheless, because of the nature of CT images in VRX-CBCT, where a stack of 2D CT slices from different layers along the axial direction are combined to form a 3D CT image, we believe that $v r x_{v u}$ is a more relevant independent variable than $\tau$ in our experiment.

Another important point that was considered when designing this experiment is the fact that based on the way the second generation VRX-CBCT is designed, the $v r x_{v u}$ is different from $v r x_{u v}$, where the sequence of rotations is reversed. Since $u$ - and $v$-axes are forming a local coordinate system that is not necessarily a simple translation from the absolute object coordinate system $x y z$, in the latter case, titling the FPD about its $u$-axis also rotates the $v$-axis that lies on the surface of the FPD. Therefore when rotating the FPD about its already rotated $v$-axis, the final result is not the same as a $v r x_{v u}$ rotation. To explain this further, assume that both $v r x_{u}$ and $v r x_{v}$ are $90^{\circ}$ and the VRX-CBCT is simplified into a conventional CBCT. We call the FPD $u$ - and $v$-axes in this position $u^{\prime}$ and $v^{\prime}$ respectively. An alternative method of achieving a $v r x_{v u}$ rotation would be to first rotate the FPD about the $v^{\prime}$ axis, and then about its current local $u$-axis, therefore by definition $v r x_{v u}=v r x_{u v^{\prime}}$ but they are both different from vr $x_{u v}$. This method of rotation was chosen to prevent any rotation about the $w$-axis of the FPD and to keep the focus of this experiment exclusively on the effect of $v r x_{u}$ and $v r x_{v}$.

\subsubsection{MTF Phantom}

A new MTF phantom was designed to measure the spatial resolution of the VRX-CBCT in both transverse and axial directions. This phantom consisted of four rectangular acrylic cubes of size $12.5 \mathrm{~mm}(\mathrm{~L}) \times 11.5 \mathrm{~mm}(\mathrm{~W}) \times 25 \mathrm{~mm}(\mathrm{H})$ labeled $a$ through $d$ (Figure 3.11a). Two $10 \mathrm{~mm} \times 10 \mathrm{~mm}$ pieces of $25 \mu \mathrm{m}$ thick copper foil were placed between the long side of the top two $(a$ and $b)$ and the bottom two $(c$ and $d$ ) acrylic cubes, and two nylon screws were used per cube pair to form two new half phantoms $a b$ and $c d$. A third piece of copper foil of size $4 \mathrm{~mm} \times 10 \mathrm{~mm}$ with the same thickness was then placed and centered between the two half phantoms, and four nylon screws were used to attach the two pieces $a b$ and $c d$ together to form the complete MTF phantom as shown in Figure 3.11b. The final size of the MTF phantom was $23 \mathrm{~mm}(\mathrm{~W}) \times 25 \mathrm{~mm}(\mathrm{~L}) \times 25 \mathrm{~mm}(\mathrm{H})$.

Since the previous stand would prevent the FPD from tilting freely in an isotropic manner, a new stand was designed for the rotary table that used a smaller acrylic tube with the external diameter of $17 \mathrm{~mm}$ to keep the same magnification ratio on the horizontal isoplane as in the previous experiment. The MTF phantom was placed on top of the new rotary table stand so that the two larger copper foils were vertical and the single smaller copper foil was almost aligned with the isoplane. The phantom 


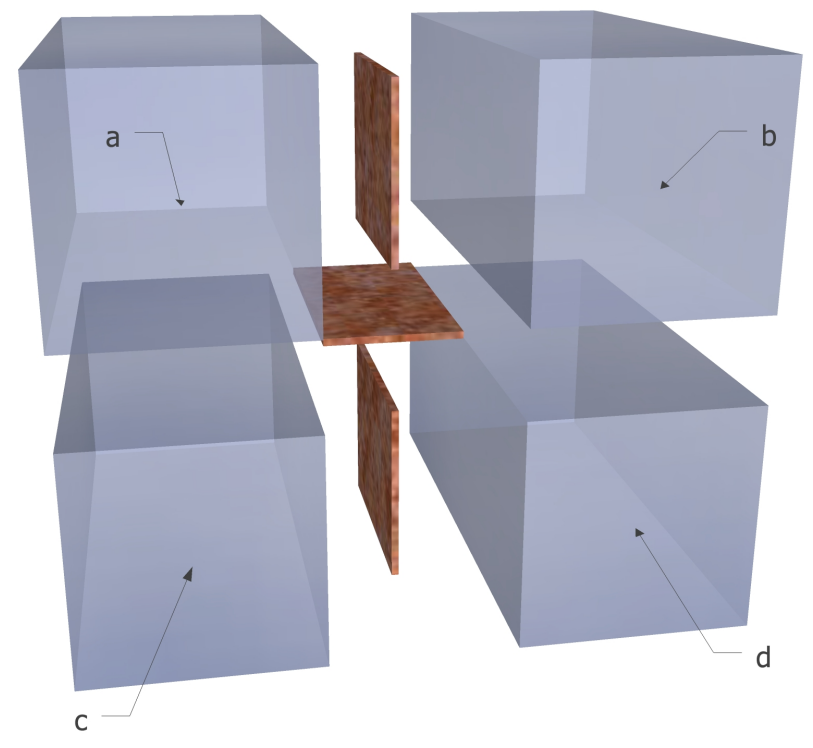

(a)

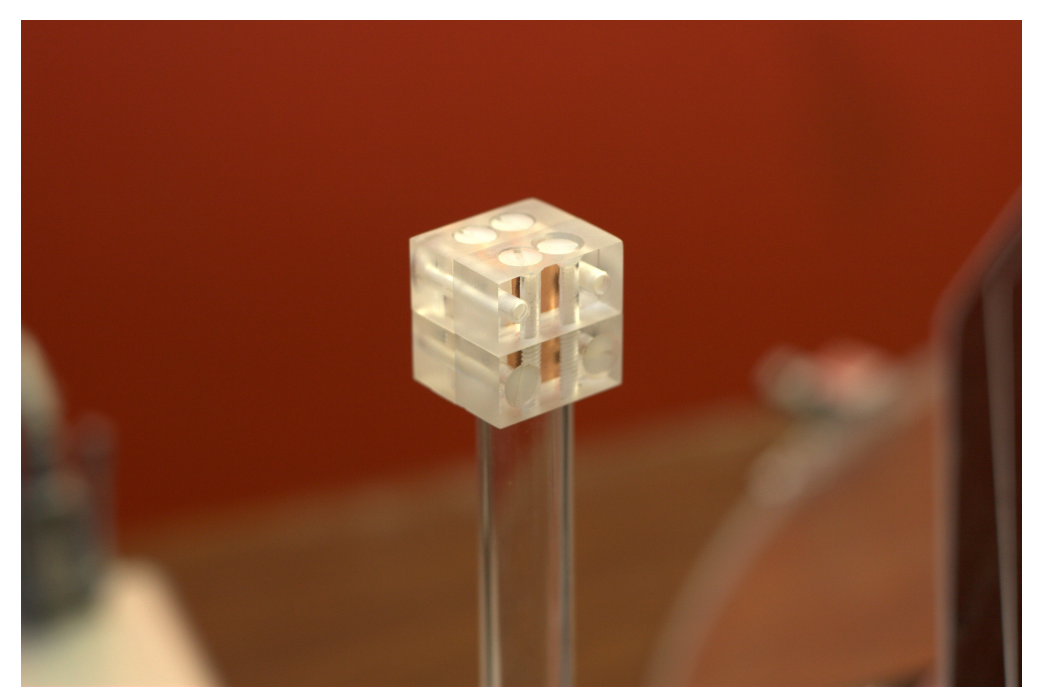

(b)

Figure 3.11: The isotropic MTF phantom.

(a) An illustration of the phantom showing two vertical and one horizontal copper foils of $25 \mu \mathrm{m}$ thickness sandwiched between four rectangular acrylic cubes $a$ through $d$, and (b) the actual MTF phantom on the new acrylic stand. 
was stabilized on the stand to prevent lateral movements, and the stand was aligned to be vertical using the same technique described earlier in this document.

\subsubsection{System Parameters}

The geometric and operating parameters of this experiment are shown in Table 3.5. For all $v r x_{v u}$ angles, the tube voltage was kept at $74 \mathrm{kVp}$ as with the previous experiment. To achieve an acceptable SNR, the tube current and the FPD frame rate were adjusted respectively from $150 \mu \mathrm{A}$ and $15 \mathrm{fps}$ for $v r x_{v u}=90^{\circ}$ to $250 \mu \mathrm{A}$ and 5 fps at $v r x_{v u}=30^{\circ}$.

\subsubsection{FPD Pre-processing and Data Acquisition}

For each $v r x_{v u}$ angle, the FPD gain calibration, offset calibration, and acquisition of the projection data were done using $\operatorname{ViVA}^{\mathrm{TM}}$ application. For $v r x_{v u} \in\left\{90^{\circ}, 60^{\circ}\right\}$, the FPD frame rate was set to $15 \mathrm{fps}$ with the rotation speed of the object rotary table set to 2.97 degrees per second $(0.052 \mathrm{rad} / \mathrm{s})$ resulting in a total scan time of $121.2 \mathrm{~s}$ (about $2 \mathrm{~min}$ ) per $360^{\circ}$. For smaller angles vr $x_{v u} \in\left\{45^{\circ}, 30^{\circ}\right\}$ however, the FPD frame rate was set to $5 \mathrm{fps}$ and the rotation speed of the rotary table was reduced to 0.99 degrees per second $(0.017 \mathrm{rad} / \mathrm{sec})$. The total scan time in the latter case was $363.6 \mathrm{~s}$ (about $6 \mathrm{~min}$ ) per $360^{\circ}$. From the total of 2048 projection images, 1818 views were selected to form a 3D sinogram.

Table 3.5: VRX-CBCT parameters for isotropic resolution analysis.

\begin{tabular}{llll}
\hline Parameter & Value & Parameter & Value \\
\hline resolution & $512 \times 512(2 \times 2$ binning $)$ & tube voltage & $74 \mathrm{kVp}$ \\
pixel pitch & $384 \mu \mathrm{m}$ & tube current & $150 \mu \mathrm{A} @ 90^{\circ}$ \\
SID & $85.5 \mathrm{~cm}$ & & $200 \mu \mathrm{A} @ 60^{\circ}$ \\
SDD & $102.0 \mathrm{~cm}$ & & $250 \mu \mathrm{A} @ 45^{\circ}$ \\
& & FPD frame rate & $250 \mu \mathrm{A} @ 30^{\circ}$ \\
& & $15 \mathrm{fps} @ 90^{\circ}$ \\
& & & $5 \mathrm{fps} @ 60^{\circ}$ \\
& & & $5 \mathrm{fps} @ 35^{\circ}$ \\
& & & $0.052 \mathrm{rad} / \mathrm{s} @ 90^{\circ}$ \\
& & & $0.052 \mathrm{rad} / \mathrm{s} @ 60^{\circ}$ \\
& & $0.017 \mathrm{rad} / \mathrm{s} @ 45^{\circ}$ \\
\hline
\end{tabular}




\subsubsection{Calibration}

A new calibration process was developed, using Matlab ${ }^{\circledR}$, to find the geometric parameters of the VRX-CBCT. The heart of the calibration algorithm is a simulation engine that can find the exact projection point on a virtual FPD for any given point in the $x y z$ object coordinate system, given the geometric parameters of the VRX-CBCT.

\subsubsection{Basic Concept}

Figure 3.12 illustrates the basic geometry used in our algorithm. The objective of the simulation algorithm is to find the coordinates of the projection point $P_{1}^{\prime}$ : $\left(x_{1}^{\prime}, y_{1}^{\prime}, z_{1}^{\prime}\right)$ of a known point $P_{1}:\left(x_{1}, y_{1}, z_{1}\right)$ in the object coordinate system and transform it to the $u v$ coordinate system of the FPD. Since $P_{1}^{\prime}$ is the intersection of the x-ray trajectory line $L_{1}$ and the FPD imaging plane $F$ :

$$
\begin{aligned}
S: & (d, 0,0) \\
B: & (d-D, 0,0) \\
\mathbf{n}: & {\left[n_{1}, n_{2}, n_{3}\right]=} \\
& {[\sin \phi \sin \psi, \cos \psi, \cos \phi], }
\end{aligned}
$$

where $d$ is SID and $D$ is SDD , $\mathbf{n}$ is a normal vector for FPD plane $F$, and $\phi$ and $\psi$ are $v r x_{u}$ and $v r x_{v}$ angles respectively. The equations for $L_{1}$ and $F$ can be written as:

$$
\begin{gathered}
F: \mathbf{n} \cdot\left(P_{1}^{\prime}-B\right)=0 \\
L_{1}: P_{1}^{\prime}=S+c\left(P_{1}-S\right) .
\end{gathered}
$$

By replacing the value of $P_{1}^{\prime}$ in the first equation with its equivalent value from the second equation, we get:

$$
\begin{gathered}
\Rightarrow \mathbf{n} \cdot\left[S+c\left(P_{1}-S\right)\right]-\mathbf{n} \cdot B=0 \\
c=\frac{\mathbf{n} \cdot(B-S)}{\mathbf{n} \cdot\left(P_{1}-S\right)} \\
=\frac{\left[n_{1}, n_{2}, n_{3}\right] \cdot[-D, 0,0]}{\left[n_{1}, n_{2}, n_{3}\right] \cdot\left[x_{1}-d, y_{1}, z_{1}\right]} \\
=\frac{n_{1} D}{n_{1}\left(d-x_{1}\right)-n_{2} y_{1}-n_{3} z_{1}} .
\end{gathered}
$$

By placing the value of $c$ back into the formula for $L_{1}$, we get :

$$
P_{1}^{\prime}=(d, 0,0)-\frac{n_{1} D}{n_{1}\left(d-x_{1}\right)-n_{2} y_{1}-n_{3} z_{1}} \cdot\left(x_{1}-d, y_{1}, z_{1}\right)
$$




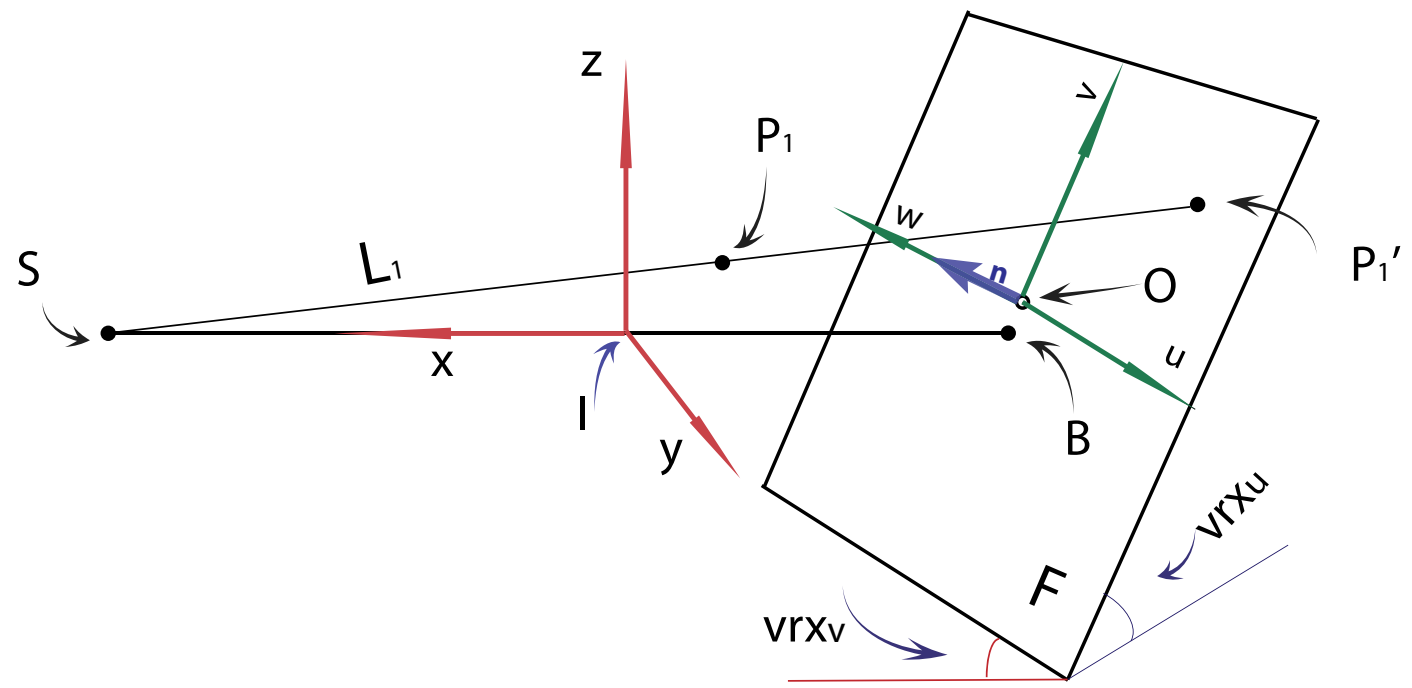

Figure 3.12: Geometry of the isotropic VRX-CBCT.

$I$ is the isocenter and the origin of the $x y z$ coordinate system and $O$ is the FPD centerpoint and the origin of the uvw coordinate system. $S$ and $B$ are the source and the base point respectively. $P_{1}$ is a known point in the $x y z$ coordinate system that is projected along line $L_{1}$ on the oblique plane $F$. The intersection of $L_{1}$ and $F$ is the projection point $P_{1}^{\prime}$. $\mathbf{n}$ is the normal vector to the plane $F$. 
and the uvw coordinates of the projection point $P_{1}^{\prime}$ can be obtained using a compound rotation and a simple translation:

$$
P_{1}^{\prime}\left(u_{1}, v_{1}, w_{1}\right)=\left[R \times P_{1}^{\prime}\left(x_{1}, y_{1}, z_{1}\right)\right]+\left(u_{0}, v_{0}, 0\right),
$$

where $u_{0}$ and $v_{0}$ are the horizontal and vertical offsets. $R$ consists of two independent rotations $R_{v}$ about the $v$-axis and $R_{u}$ about $v$-axis such that:

$$
\begin{aligned}
R & =R_{v} \times R_{u} \\
& =\left[\begin{array}{ccc}
\cos \left(\frac{\pi}{2}-\phi\right) & -\sin \left(\frac{\pi}{2}-\phi\right) & 0 \\
\sin \left(\frac{\pi}{2}-\phi\right) & \cos \left(\frac{\pi}{2}-\phi\right) & 0 \\
0 & 0 & 1
\end{array}\right] \times\left[\begin{array}{ccc}
\cos \left(\frac{\pi}{2}-\psi\right) & 0 & -\sin \left(\frac{\pi}{2}-\psi\right) \\
0 & 1 & 0 \\
\sin \left(\frac{\pi}{2}-\psi\right) & 0 & \cos \left(\frac{\pi}{2}-\psi\right)
\end{array}\right] \\
& =\left[\begin{array}{ccc}
\sin \phi & -\cos \phi & 0 \\
\cos \phi & \sin \phi & 0 \\
0 & 0 & 1
\end{array}\right] \times\left[\begin{array}{ccc}
\sin \psi & 0 & -\cos \psi \\
0 & 1 & 0 \\
\cos \psi & 0 & \sin \psi
\end{array}\right] .
\end{aligned}
$$

Using the rotation matrix $R$, the $w$ component of the projection point $P_{1}^{\prime}$ is always zero, leaving $P_{1}^{\prime}$ on the surface of the virtual FPD imaging plane $F$ as expected. Therefore $P_{1}^{\prime}$ can now be uniquely identified in the $u v$ coordinate system of the FPD using the notation $\left(u_{1}^{\prime}, v_{1}^{\prime}\right)$.

Now assume that we have an infinitesimal object with infinite linear attenuation coefficient located on the VRX-CBCT rotary table. The exact location of this point in the object coordinate system is known to be $(x, y, z)$, however the exact values of the geometric parameters SID, SDD, vr $x_{u}, v r x_{v}$, and the horizontal and vertical offset values $\left(u_{0}, v_{0}\right)$ are not immediately known and only their approximate values are available. The procedure above can calculate the projection point $\tilde{E}^{\prime}:\left(u_{E^{\prime}}, v_{E^{\prime}}\right)$ based on the available information (i.e., the best estimated values). If the estimates for the geometric parameters are correct, $\tilde{E}^{\prime}$ should fall exactly on the actual projection $P^{\prime}$. However in practice these two points are almost always separate from each other. If the scalar value $e$ indicates the error between these two points in the $u v$ coordinate system, then:

$$
e^{2}=\left|\overrightarrow{P^{\prime}}-\overrightarrow{\tilde{E}^{\prime}}\right|^{2}=\delta_{u}^{2}+\delta_{v}^{2}
$$

A minimization process can be used to find the best independent variables (i.e., the geometric parameters SID, SDD, vr $x_{u}, v r x_{v}, u_{0}$ and $v_{0}$ ) to bring the two points $\tilde{E}^{\prime}$ and $P^{\prime}$ closer to each other to the point where $e^{2} \leq \epsilon^{2}$ where $\epsilon$ is the maximum acceptable error.

\subsubsection{Implementation}

A new phantom was developed for the $3 \mathrm{D}$ calibration process. The phantom consists of 15 steel ball bearings of $1.6 \mathrm{~mm}\left(1 / 16^{\prime \prime}\right)$ diameter attached along the side of a $23 \mathrm{~cm}$ long acrylic tube of $17 \mathrm{~mm}$ outer diameter as shown in Figure 3.13. The 


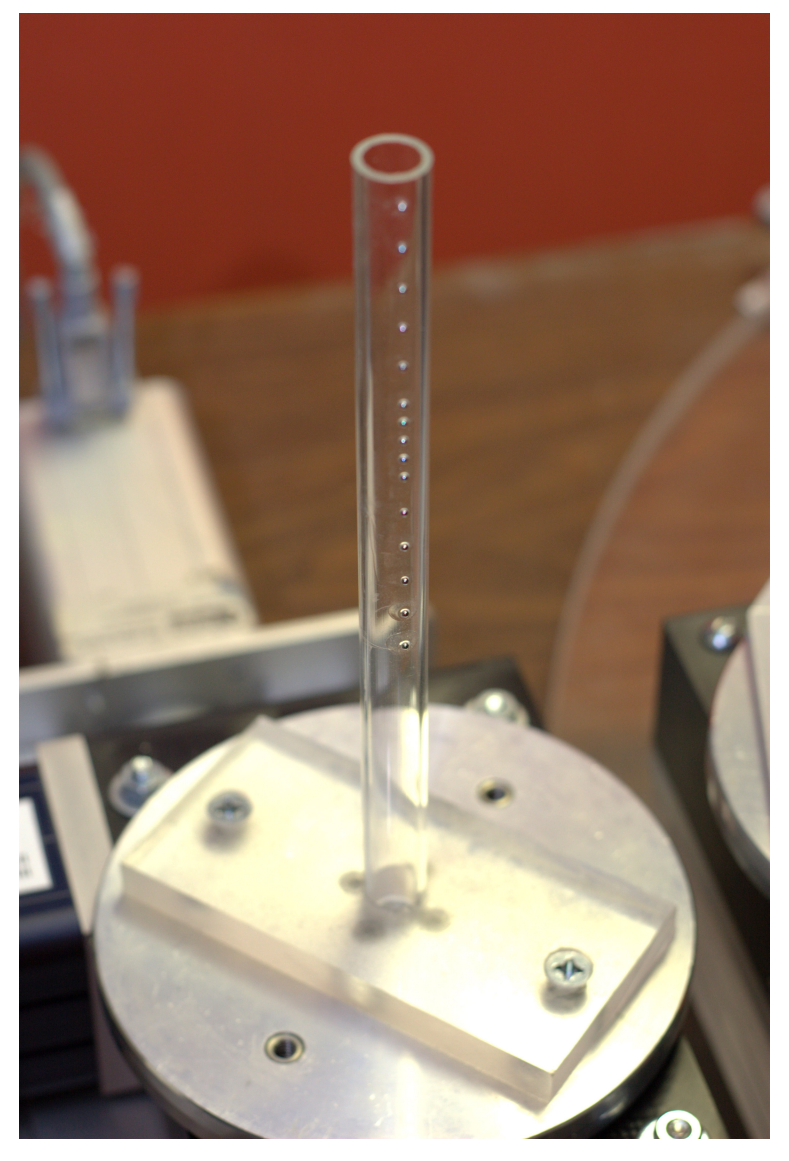

Figure 3.13: 3D calibration phantom.

The phantom consists of 15 steel ball bearings of size $1.6 \mathrm{~mm}$ attached to the outer surface of a $23 \mathrm{~cm}$ long acrylic tybe of $17 \mathrm{~mm}$ outer diameter. 
center ball was placed at a height where it was estimated to fall approximately on the isoplane. Then 12 more balls were placed symmetrically on both sides of the center ball at $10 \mathrm{~mm}$ intervals. The remaining 2 balls were attached between each pair of the three central balls, to form a central region of 5 balls at $5 \mathrm{~mm}$ intervals. All balls were designed to fall on a single line parallel to the central axis of the acrylic tube.

The phantom was placed on the rotary table and aligned to be vertical. A full $360^{\circ} \mathrm{CT}$ scan of the phantom was obtained containing $18182 \mathrm{D}$ projection images. The algorithm selected $n$ frames $f_{0}$ to $f_{n-1}$ out of the complete projection image sequence $\mathcal{F}$ so that:

$$
f_{i} \in\left\{f_{\theta} \mid \theta=\theta_{0}+\frac{i}{n} \times 2 \pi, i=0 \ldots n-1\right\} \subset \mathcal{F},
$$

where $\theta$ is the phase value of the rotary table and $\theta_{0}$ is the initial phase. Each $f_{i}$ contains the complete information about the location $\left(u_{i}, v_{i}\right)$ of the center of intensity of the projection points $P_{i}^{\prime}$ for all - or a subset of - the 15 steel balls $P_{i}$ on the phantom. Using a histogram-based adaptive thresholding filter and center of intensity analysis, the locations of the projection points for all the visible balls $\left\{P_{i}^{\prime}:\left(u_{i}^{\prime}, v_{i}^{\prime}\right) \mid i=1 \ldots k, k \leq 15\right\}$ were obtained and stored. By calculating the relative distances between visible balls, the projection $P_{c}^{\prime}$ of the central ball was identified in the set and used as a reference point for the minimization algorithm to match the corresponding location sets.

The algorithm then steps into its next stage, where it attempts to simulate the projection points of a set of 15 infinitesimal imaginary balls $E_{j}$, with the same geometric properties of the calibration phantom, to a virtual FPD tilted to the $\tilde{\phi}$ and $\psi$ angles, the estimated values of $v r x_{u}$ and $v r x_{v}$ respectively. Five other estimates of the geometric parameters of the system, namely $\tilde{D}^{\prime}, \tilde{d}^{\prime}, \tilde{u}_{0}, \tilde{v}_{0}$, and the initial phase $\tilde{\theta}_{0}$ were also used in the simulation process. The result was a set of 15 point locations on the $u v$ coordinate system $\left\{\tilde{E}_{j}^{\prime}:\left(\tilde{u}_{E_{j}^{\prime}}, \tilde{v}_{E_{j}^{\prime}}\right) \mid j=1 \ldots 15\right\}$.

The two points $P_{c}^{\prime}$ and $\tilde{E}_{8}^{\prime}$, which were used as the reference points on each set were matched first. Then remaining simulated points $\left\{\tilde{E}_{j}^{\prime}\right\}$ that were missing a counterpart in the actual projection point set $\left\{P_{i}^{\prime}\right\}$ were dismissed from the set. The result was a set of estimated projection points $\left\{\tilde{E}_{i}^{\prime} \mid i=1 \ldots k, k \leq 15\right\}$, where each element of it corresponded to one and only one element in the $\left\{P_{i}^{\prime}\right\}$ set. The normalized sum of square errors (NSSE) was then calculated using the following formula:

$$
\mathrm{NSSE}=\frac{1}{k} \sum_{i=1}^{k} e_{i}^{2}=\frac{1}{k} \sum_{i=1}^{k}\left(\left(\tilde{u}_{E_{i}}-u_{i}\right)^{2}+\left(\tilde{v}_{E_{i}}-v_{i}\right)^{2}\right) .
$$

The algorithm uses an iterative simplex method [70], implemented in Matlab's fminsearch function, to find the geometric parameters $\operatorname{vr} x_{u}, v r x_{v}$, SSD, SID, $u_{0}$, $v_{o}$, and $\theta_{0}$ that satisfy the minimization conditions, i.e., when either the function 
tolerance or the geometric parameters tolerance was less than $10^{-7}$, or if the number of iterations simply exceeded 2000 .

There are two important problems that need to be addressed here. First, the number of projection image frames $n$ selected for the calibration process should be large enough to prevent the minimization algorithm from falling to a local minimum. At the same time, our experience with large n's shows that the matched simulated points tend to be skewed toward outer region balls, leaving relatively larger partial NSSE in the central region of the calibration phantom compared to that of fewer frames. We believe that a reasonable range for $n$ is any odd number between 5 and 30. In our experiments, $n$ was chosen to be 7 .

The second problem to address is the effectiveness of the simplex minimization algorithm when the initial estimated geometric parameters are relatively far away from the actual values. In the simplex algorithm, all $n+1$ points in the $n$-dimensional space have the same priority when finding the minimum error function, whereas in reality some geometric parameters are more sensitive than the others. This characteristic of the minimization algorithm increases the probability of it falling into local minima. To prioritize the variables, the minimization was done in three stages. Figure 3.14 shows the three different stages of NSSE minimization in a VRX-CBCT calibration run for $v r x_{v u}=30^{\circ}$. In the first stage only $\theta_{0}, u_{0}$, and $v_{0}$ were optimized. The result was a new set of best estimates for these geometric parameters that were fed to the minimization algorithm in the second stage along with two more parameters vr $x_{u}$ and $v r x_{v}$. In the third stage, all seven geometric parameters were optimized, with the first five derived directly from the result of the second phase. In our experiments, this method proved to increase the overall effectiveness of the $3 \mathrm{D}$ calibration process.

\subsubsection{CT Reconstruction and MTF Calculation}

\subsubsection{CT Reconstruction Algorithm}

The new geometry of the system prevents us from using a simple FDK algorithm efficiently. The previous VRX-CBCT reconstruction algorithms relied on preconditioning the projection images either along the $u$-axis or the $v$-axis of the FPD. Although possible to implement, a simple FDK algorithm that takes advantage of $2 \mathrm{D}$ preconditioning of the projection images may introduce even larger errors in the final 3D CT image. The assumptions to simplify the geometry for a typical algorithm may not apply especially at small $v r x_{v u}$ angles. For this reason, a new approach to reconstruct 3D CT images using the VRX-CBCT system was deemed necessary.

The new VRX-CBCT reconstruction algorithm is a voxel-driven method, meaning that the intensity value of each voxel in the reconstruction cube is independently calculated from a single cell, or by interpolation between a group of cells, on the FPD projection data matrix. In the alternative method called ray-driven, each detector cell on the FPD contributes to the intensity values of several voxels along the projec- 


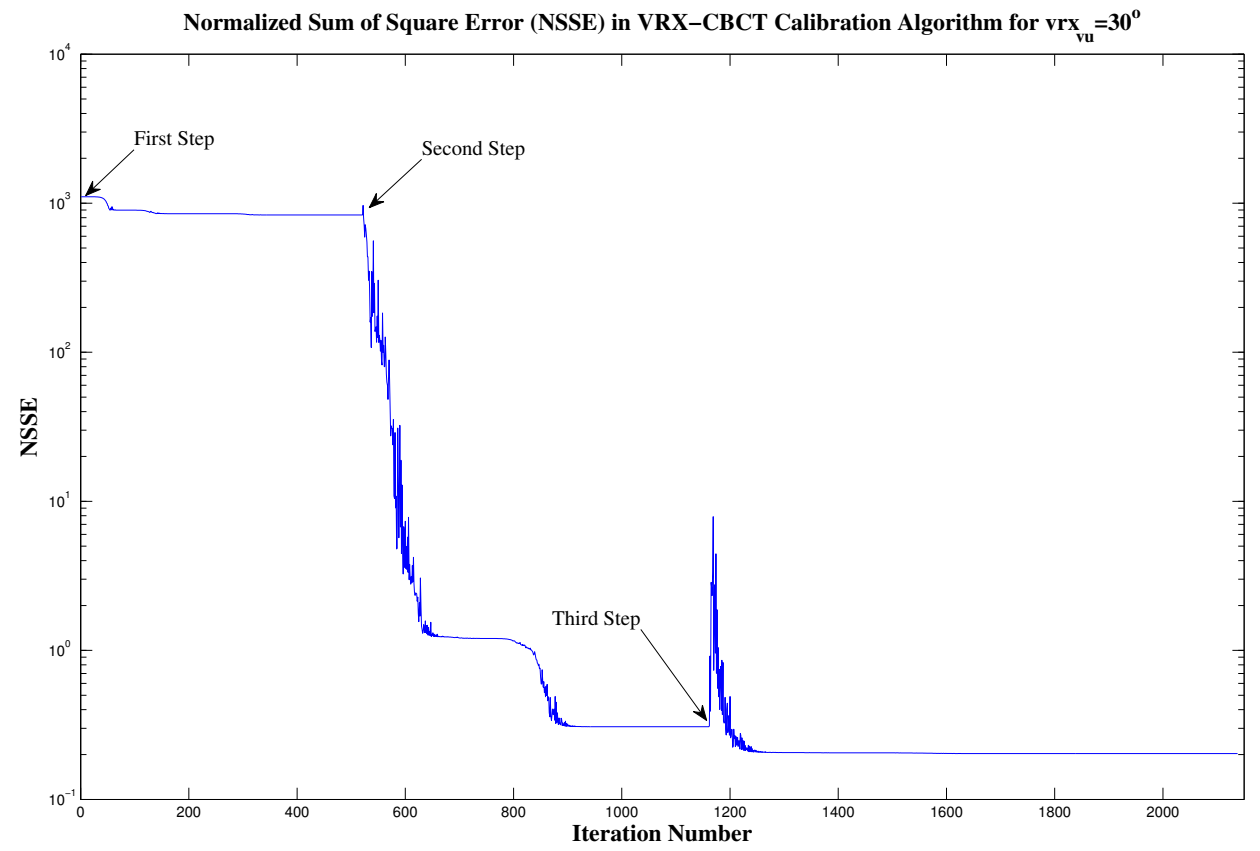

Figure 3.14: NSSE three-stage minimization.

System geometrical parameters $\theta_{0}, u_{0}$, and $v_{0}$ were optimized in the first stage. In the second stage, these calibrated parameters were minimized along with vr $x_{u}$ and vr $x_{v}$. The calibration algorithm then minimizes all seven system geometric parameters (the first five plus SID and SDD) in the third stage. 
tion line. Although these two methods should result in similar reconstruction images, choosing a voxel-driven method for backprojection in VRX-CBCT would eliminate the non-linear signal intensity aggregation caused by the increase in the angular density of cells (i.e., number of contributing cells to a single voxel in a unit of angle) going toward the far side of the FPD. Moreover, since we already had a simulation engine that could find the projection location of known points in the object coordinate system, a voxel-driven approach was simply more practical to implement.

In the first step of the new VRX-CBCT reconstruction algorithm, a 3D matrix representing a virtual cube of $n(\mathrm{~L}) \times m(\mathrm{~W}) \times l(\mathrm{H})$ voxels of size $\sigma_{n} \times \sigma_{m} \times \sigma_{l} \mathrm{~mm}^{3}$ was created in the computer memory. The geometry of the cube was set so that the axis of rotation would pass through the center of the cube's $n \times m$ rectangular sides. The location of the voxels' center point $\left\{\left(x_{i j k}, y_{i j k}, z_{i j k}\right)\right\}$ was then calculated based on the following formula:

$$
\left(x_{i j k}, y_{i j k}, z_{i j k}\right)=\left[\begin{array}{ccc}
\sigma_{n} & 0 & 0 \\
0 & \sigma_{m} & 0 \\
0 & 0 & \sigma_{l}
\end{array}\right] \times\left[\begin{array}{c}
\frac{i-n}{2} \\
\frac{j-m}{2} \\
\frac{k-l}{2}
\end{array}\right], \quad\left\{\begin{array}{l}
i=1 \ldots n \\
j=1 \ldots m \\
k=1 \ldots l
\end{array} .\right.
$$

The simulation engine described in section 3.5.3.1 was used to project the center of all voxels in the cube onto a virtual FPD using the geometric parameters provided by the calibration method. The algorithm would then calculate the intensity values of the voxels $I_{i j k}$ in each specific view $f_{\theta}$, using bilinear interpolation over the values of a group of 4 adjacent cells in the vicinity of the location of the projection points. After all voxels were filled with the proper intensity values $I_{i j k}$, the cube, which now represents a $3 \mathrm{D}$ backprojected image of a single view $f_{\theta}$, was first normalized and then rotated about the $z$-axis to match the view angle $\theta$. The algorithm repeated the procedure for each remaining frame $f_{\theta}$ and added the result of the rotated cube after each iteration to the first cube. The final 3D image was complete when the algorithm had processed all the frames that would constitute a full $360^{\circ} \mathrm{VRX}-\mathrm{CBCT}$ scan.

\subsubsection{MTF Calculations}

The MTF phantom described in section 3.5.1.2 was placed on the rotary table stand within the FOV of the tilted FPD such that the center of the MTF phantom was approximately on the isoplane. Four isotropic vr $x_{v u}$ angles of $90^{\circ}, 60^{\circ}, 45^{\circ}$, and $30^{\circ}$ were selected for this experiment. For each $v r x_{v u}$ angle in the experiment, five sets of projection images of the MTF phantom were acquired. A 3D CT image (cube of voxels) of size $20 \mathrm{~mm}(\mathrm{~L}) \times 20 \mathrm{~mm}(\mathrm{~W}) \times 10 \mathrm{~mm}(\mathrm{H})$ with $50 \mathrm{\mu m}^{3}$ voxel size was obtained from 700 views selected from a total of 1818 series of $2 \mathrm{D}$ projection data, using bilinear interpolation and Shepp-Logan filtration. The LSF and MTF values for each isotropic $v r x_{v u}$ angle were then calculated using the technique described in section 3.4.4.2.

In addition to finding the spatial resolution characteristics of the isotropic VRX$\mathrm{CBCT}$, we used the $3 \mathrm{D}$ reconstruction algorithm on the projection images taken at 
several non-isotropic $\operatorname{vr} x_{v}$ and vr $x_{u}$ angles. The results were then compared to those of the isotropic system. 


\section{Chapter 4}

\section{Results and Discussion*}

In this chapter, we present the results of the spatial resolution analysis of the variable resolution x-ray cone-beam CT (VRX-CBCT) system. The line spread function (LSF), representing the resolution of the system in the spatial domain, and the modulation transfer function (MTF), representing the resolution of the system in the frequency domain were calculated using the custom-made MTF phantoms described in Chapter 3. Two components of spatial resolution of the system, namely the transverse and axial spatial resolution were analyzed in three phases. In phase one, $\mathrm{LSF}_{\text {trans }}$ and $\mathrm{MTF}_{\text {trans }}$ were measured for the VRX-CBCT system when the flat panel detector (FPD) was tilted only about its vertical axis $(v)$. In the second phase, $\mathrm{LSF}_{\text {axial }}$ and $\mathrm{MTF}_{\text {axial }}$ were measured in the second generation VRX-CBCT system when the FPD was tilted only about its horizontal axis $(u)$. In the third phase of the study, both transverse and axial spatial resolution components in spatial and frequency domains were analyzed in an isotropic VRX-CBCT using 3D CT images produced by a new reconstruction algorithm, when the FPD tilted about both its $u$ and $v$-axes in an isotropic manner - i.e., when both rotation angles $v r x_{v}$ and $v r x_{u}$ were the same. In addition, the effectiveness of the new VRX-CBCT reconstruction algorithm was measured by repeating the $\mathrm{MTF}_{\text {trans }}$ and $\mathrm{MTF}_{\text {axial }}$ measurements of the first two phases of the study using the new algorithm and comparing the results to those with the algorithms previously used.

\subsection{Transverse Spatial Resolution of the First Generation VRX- CBCT}

Figure 4.1a illustrates the reconstructed VRX-CBCT images of the central slice of the MTF phantom taken at vrx $x_{v}$ angles of $90^{\circ}, 65^{\circ}, 45^{\circ}$, and $30^{\circ}$. It can be observed that the width of the CT image of the copper foil gets thinner as the vr $x_{v}$ angle decreases. This result suggests that the combined effect of scattering of light

* Section 4.1 of this chapter is adapted with permission from Dahi et al., "Performance analysis of a CsI-based flat panel detector in a cone beam variable resolution x-ray system", SPIE - The International Society for Optical Engineering, vol. 6510, pp. 65104B-1 - 65104B-8, 2007.

Section 4.2 is adapted with permission from Dahi et al., "Analysis of axial spatial resolution in a variable resolution x-ray cone beam CT (VRX-CBCT) system", SPIE - The International Society for Optical Engineering, vol. 69134, pp. 69134Y-1 - 69134Y-8, 2008. 


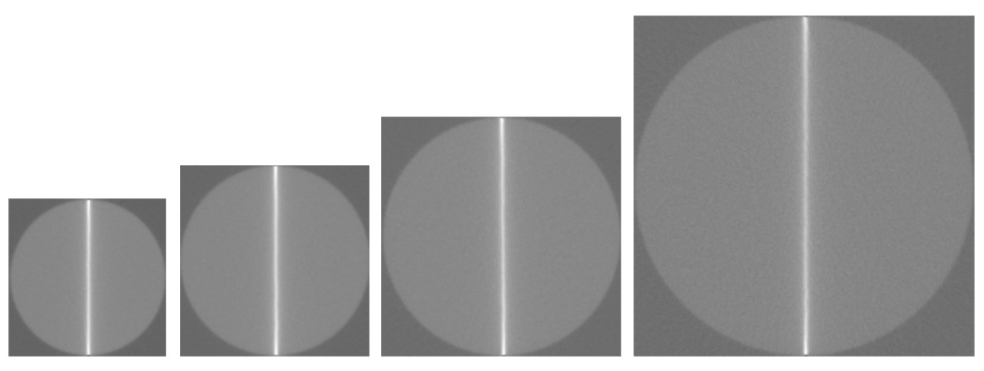

(a)

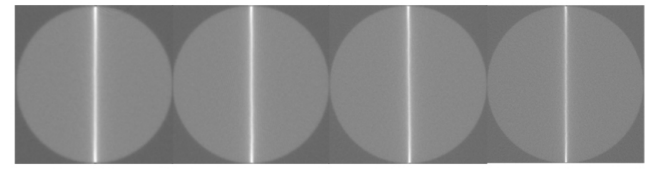

(b)

Figure 4.1: VRX-CBCT images taken at different $v r x_{v}$ angles.

The images are taken at $90^{\circ}$ (far left), $65^{\circ}, 45^{\circ}$, and $30^{\circ}$ (far right) (a) in their actual size, and (b) resized to match the dimensions of the image taken at $\operatorname{vr} x_{v}=90^{\circ}$. 
photons in the phosphor and the trajectory of the x-ray across multiple detectors are outweighed by the VRX effect in the VRX-CBCT system. The images taken with smaller $v r x_{v}$ angles are resized in Figure $4.1 \mathrm{~b}$ to match the one taken at $90^{\circ}$, which is equivalent to conventional CBCT. The improvement in spatial resolution can be seen when the $v r x_{v}$ angle decreases from $90^{\circ}$ (far left) to $30^{\circ}$ (far right).

The MTF curves of the VRX-CBCT system at different $v r x_{v}$ angles are shown in Figure 4.2a. The sharp drop in all of the MTF curves is associated with the overall low MTF of the indirect FPD as well as the relatively large sampling distance of about $0.4 \mathrm{~mm}$ that corresponds to a cut-off frequency of $2.5 \mathrm{~mm}^{-1}$ in the projection mode. Table 4.1 lists the values of spatial frequencies corresponding to MTF values of 0.1 and 0.2 as well as the MTF values at spatial frequencies of $0.5,1.0$, and $1.5 \mathrm{~mm}^{-1}$ for all four VRX angles chosen for the experiment. Overall it can be seen that the MTF for the VRX-CBCT system increases as the VRX angle decreases despite the increasing lateral spreading of the detected energy clouds caused by the increasing lateral penetration of the x-ray photons. To demonstrate this improvement more clearly, the same MTF curves in Figure 4.2a are shown with logarithmic scale in Figure 4.2b. All curves show a smooth behavior down to about $1 \%$ MTF. The odd shape of the MTF curves below this value could be caused by the high frequency acquisition noise and CT reconstruction artifacts. For practical purposes, the spatial frequencies at $1 \% \mathrm{MTF}$ are used to compare the cut-off frequencies of the MTF curve at each $v r x_{v}$ angle presented. The results indicate that for the $v r x_{v}$ angle of $30^{\circ}$ compared to the conventional CBCT, the system MTF cut-off frequency has improved by a factor of $2.5 / 1.5=1.67$ with the standard deviation of \pm 0.007 . This value is approximately $16.5 \%$ less than the theoretical value of $\sin 90^{\circ} / \sin 30^{\circ}=2.0$ expected for the experiment. This reduction in VRX effectiveness may be justified by the characteristics of the FPD described earlier.

Figure 4.3 shows an actual CT slice of a $10 \mathrm{~mm}$ beta-tricalcium phosphate scaffold (TCP) taken with the VRX-CBCT system at a $v r x_{v}$ angle of $10^{\circ}$. At this angle, the effective sampling distance is approximately 5.8 times smaller than the sampling distance at $90^{\circ}$. Although this sampling improvement does not completely translate into improvement in spatial resolution, it can be seen from Figure $4.3 \mathrm{~b}$ that it yields reasonable detail.

\subsection{Axial Spatial Resolution of the Second Generation VRX- CBCT}

The image of the thin copper foil in the central sagittal slice of the phantom at vr $x_{u}$ angle of $20^{\circ}$ shows significantly better resolution than that of vr $x_{u}=90^{\circ}$ (Figure 4.4). Compared qualitatively to the results from the previous section, the VRX effect on the axial direction seems to be more efficient than in the transverse direction.

MTF curves at different $v r x_{u}$ angles are shown in Figure 4.5. The standard error 


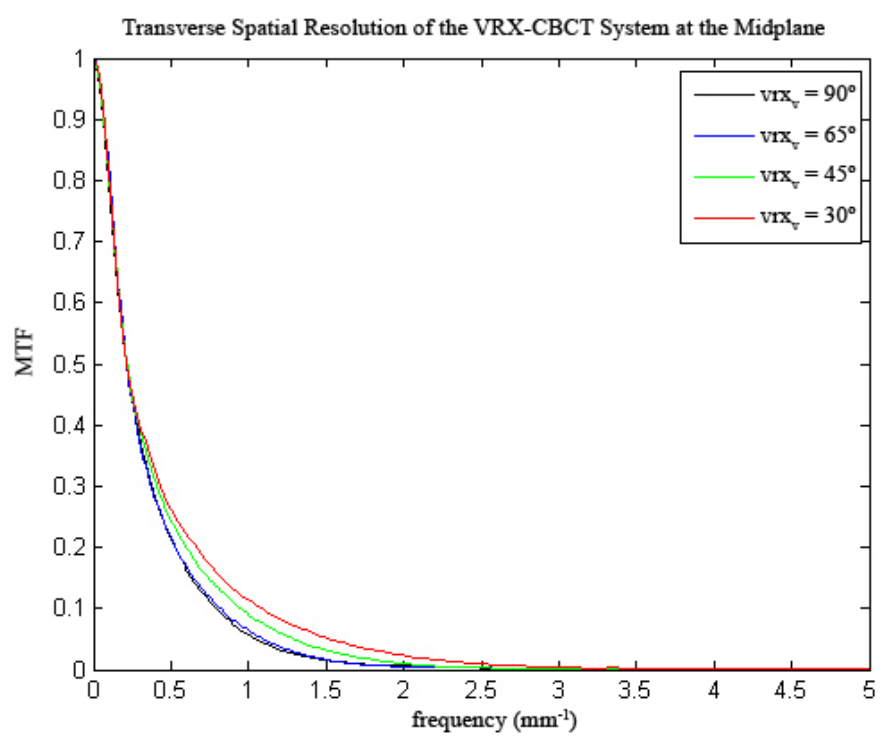

(a)

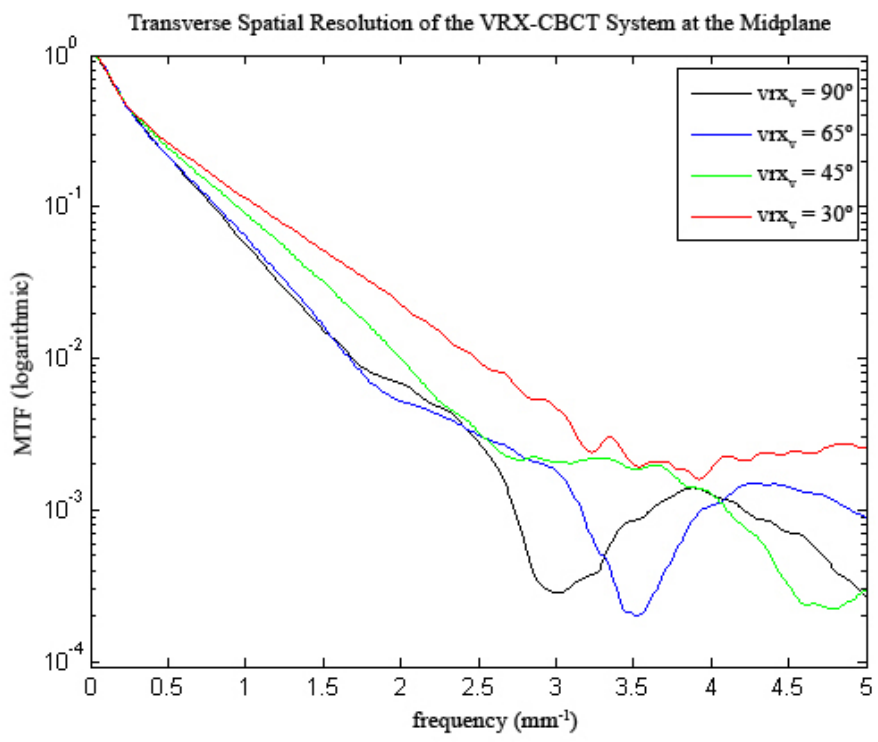

(b)

Figure 4.2: Transverse system MTF curves of the VRX-CBCT.

(a) Transverse MTF curves in normal scale, and (b) transverse MTF curves in semi logarithmic scale. 
Table 4.1: Selected frequencies and MTF values of the VRX-CBCT system.

\begin{tabular}{ccccc}
\hline & vrx $_{v}=90^{\circ}$ & vrx $_{v}=65^{\circ}$ & vr $x_{v}=45^{\circ}$ & vr $x_{v}=30^{\circ}$ \\
\hline spatial frequencies & & & & \\
@ 0.2 MTF & $0.53 \mathrm{~mm}^{-1}$ & $0.54 \mathrm{~mm}^{-1}$ & $0.60 \mathrm{~mm}^{-1}$ & $0.66 \mathrm{~mm}^{-1}$ \\
@ 0.1 MTF & $0.79 \mathrm{~mm}^{-1}$ & $0.82 \mathrm{~mm}^{-1}$ & $0.95 \mathrm{~mm}^{-1}$ & $1.08 \mathrm{~mm}^{-1}$ \\
MTF values & & & & \\
@ 0.5 mm & & & & \\
@ $1.0 \mathrm{~mm}^{-1}$ & $0.217 \pm 0.005$ & $0.217 \pm 0.013$ & $0.246 \pm 0.004$ & $0.264 \pm 0.006$ \\
$@ 1.5 \mathrm{~mm}^{-1}$ & $0.016 \pm 0.005$ & $0.064 \pm 0.008$ & $0.090 \pm 0.004$ & $0.114 \pm 0.004$ \\
\end{tabular}




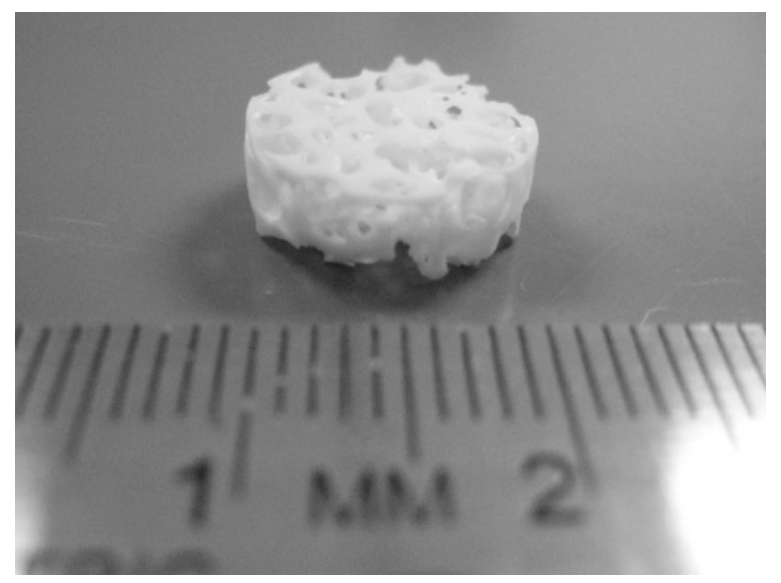

(a)

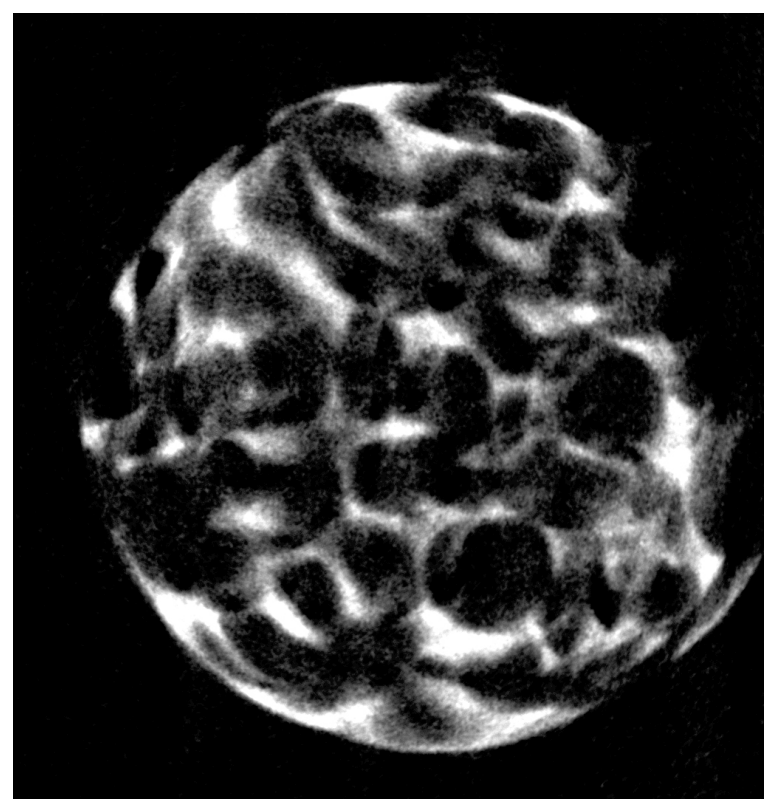

(b)

Figure 4.3: A VRX-CBCT image of a piece of beta-tricalcium phosphate scaffold (TCP).

(a) The actual specimen and (b) its VRX-CBCT image taken at $v r x_{v}$ angle of $10^{\circ}$. TCP specimen courtesy of Dr. Yunzhi Yang, University of Texas Health Science Center, Houston, TX. 
Figure 4.4: Sagittal views of the axial MTF phantom.

(a) At vr $x_{u}=90^{\circ}$, and (b) at vr $x_{u}=20^{\circ}$. The image taken at vr $x_{u}$ angle of $20^{\circ}$ shows a significant resolution improvement over the image taken at vr $x_{u}$ of $90^{\circ}$.

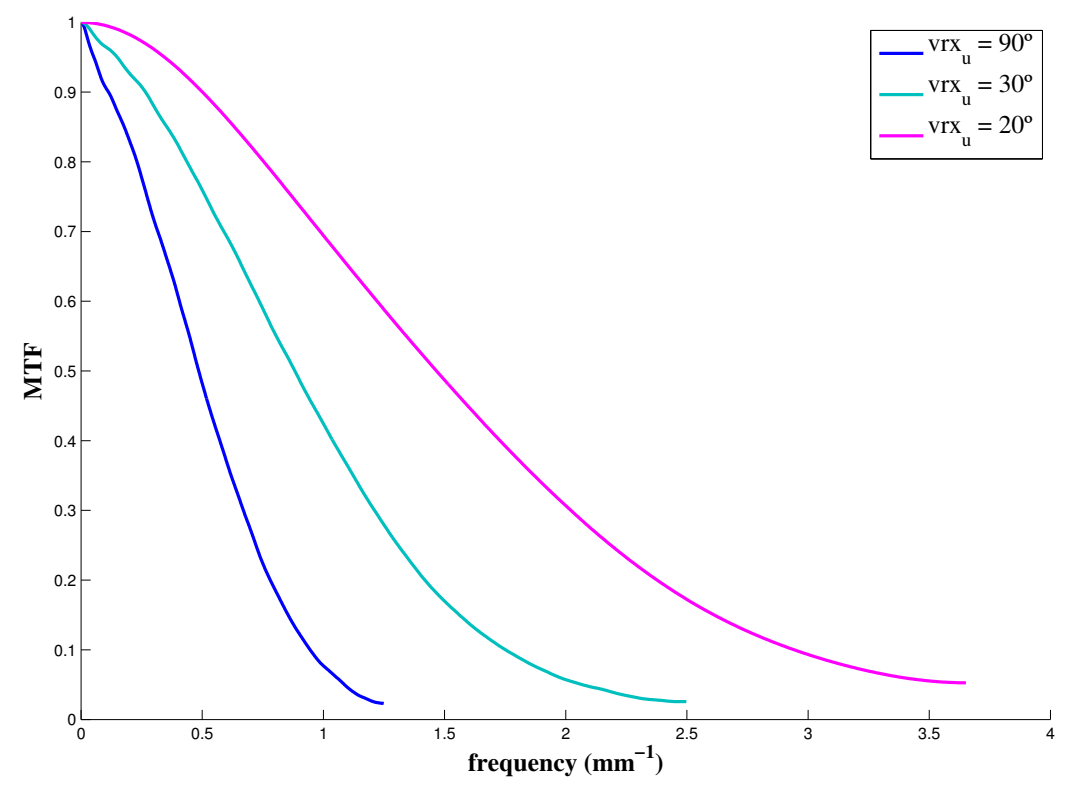

Figure 4.5: Axial spatial resolution of the VRX-CBCT system at various vr $x_{u}$ angles.

There is a significant improvement in the spatial resolution of the VRX-CBCT system at small $v r x_{u}$ angles comparing to that of $v r x_{u}=90^{\circ}$. The curves do not cross the frequency axis, suggesting that the number of samples per LSF is smaller than an optimal value. 
of the mean for each experiment is in the order of $10^{-2}$ or less, and thus the error bars are omitted from the graph. It can be seen that the MTF curve of the VRX-CBCT is significantly higher when the system is set at the smallest vr $x_{u}$ angle of $20^{\circ}$. The curves do not cross the frequency axis, suggesting that perhaps the number of samples per LSF is smaller than an optimal value. It is possible that the MTF curves include the effect of aliasing, hence showing higher values of modulation than anticipated, toward the high frequencies. Comparison of the frequencies corresponding to the $10 \%$ value of MTF for vr $x_{u}$ angles of $90^{\circ}$ and $30^{\circ}$ in Figure 4.5 shows a resolution improvement of $1.75 / 0.95=1.84$. This ratio is about $34 \%$ higher than the corresponding frequency ratio $1.08 / 0.79=1.37$ at $10 \%$ of MTF in the previous experiment (Table 4.1).

Figure 4.6 shows CT images of a slightly stretched metal coiled spring. The center-to-center distance between adjacent rings was adjusted to be slightly smaller than the FPD pixel pitch at $v r x_{u}$ angle of $90^{\circ}$, while being slightly larger than the projected pixel pitch of the FPD at $30^{\circ}$. These images show that the coiled spring is not fully resolved at $\operatorname{vr} x_{u}$ angle of $90^{\circ}$, whereas at $\operatorname{vr} x_{u}=30^{\circ}$ most of the coil rings

are distinctly visible in the CT image. Using smaller vr $x_{u}$ angle also helped reduce aliasing, which was distorting the image with repetitive patterns.

\subsection{Comprehensive Spatial Resolution of the Isotropic VRX- CBCT}

Figure 4.7 compares CT reconstructed images of the MTF phantom midplane at two isotropic $v r x_{v u}$ angles of $90^{\circ}$ and $30^{\circ}$ and two $v r x_{v}$ angles of $30^{\circ}$ and $15^{\circ}$. The top three images show that the transverse spatial resolution of the VRX-CBCT system improves as the vr $_{v}$ angle decreases. This result is similar to the qualitative result in the first phase of the study in which an FDK-based algorithm was used to reconstruct the CT images. However, when comparing the two isotropic images at $v r x_{v u}=90^{\circ}$ and $v r x_{v u}=30^{\circ}$ with the CT image taken at $v r x_{v}=30^{\circ}$, it can be seen that the VRX effect for the CT image taken at isotropic angle $v r x_{v u}=30^{\circ}$ is significantly less than the VRX effect at $v r x_{v}=30^{\circ}$.

Figure 4.8 shows quantitative measurement for the transverse spatial resolution of the VRX-CBCT system at various $v r x_{v}$ angles. It can be seen that in general the MTF values increase as the vr $x_{v}$ angle decreases. This result is comparable to our findings in Section 4.1. Differences between the MTF curve at vr $x_{v}=15^{\circ}$ and the curve at vr $_{v}=90^{\circ}$ are statistically significant considering the maximum standard deviation of the mean for all MTF curves at any points did not exceed 0.0042 .

Although no major changes to the spatial resolution in the axial direction were expected when the FPD was tilted about its $v$-axis, the axial MTF curves were still calculated for each $v r x_{v}$ angle (Figure 4.9). In theory, there should not be any difference between the MTF curves presented in this figure. The small variations in the MTF curves are assumed to be partially associated with under-sampling, due 


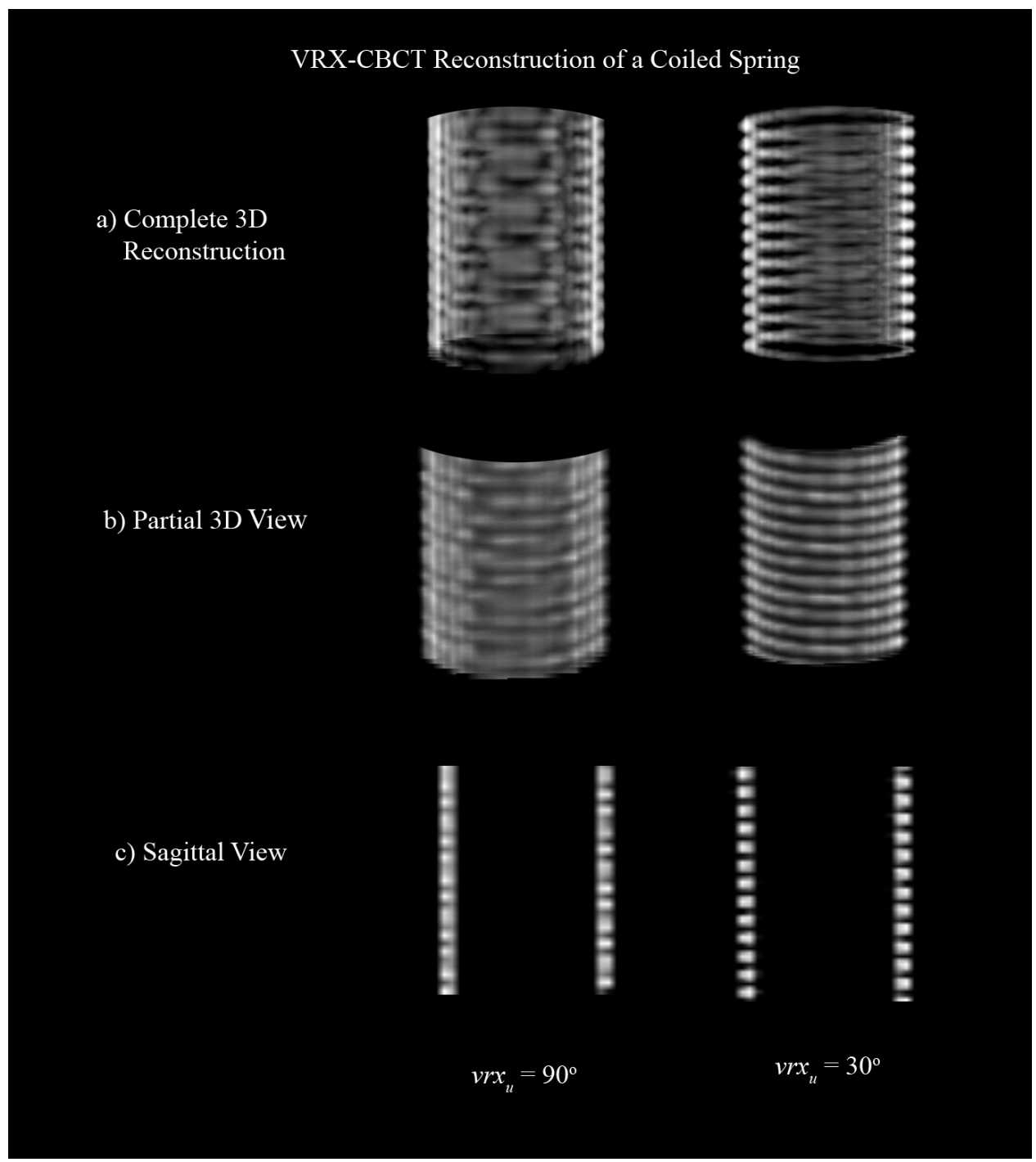

Figure 4.6: Reconstructed CT images of a slightly stretched coiled spring. (a) Full 3D views, (b) partial 3D views, and (c) sagittal view at two vr $x_{u}$ angles of $90^{\circ}$ and $30^{\circ}$. The images taken at vr $_{u}$ of $30^{\circ}$ are resolved more effectively than the images taken at $90^{\circ}$. 

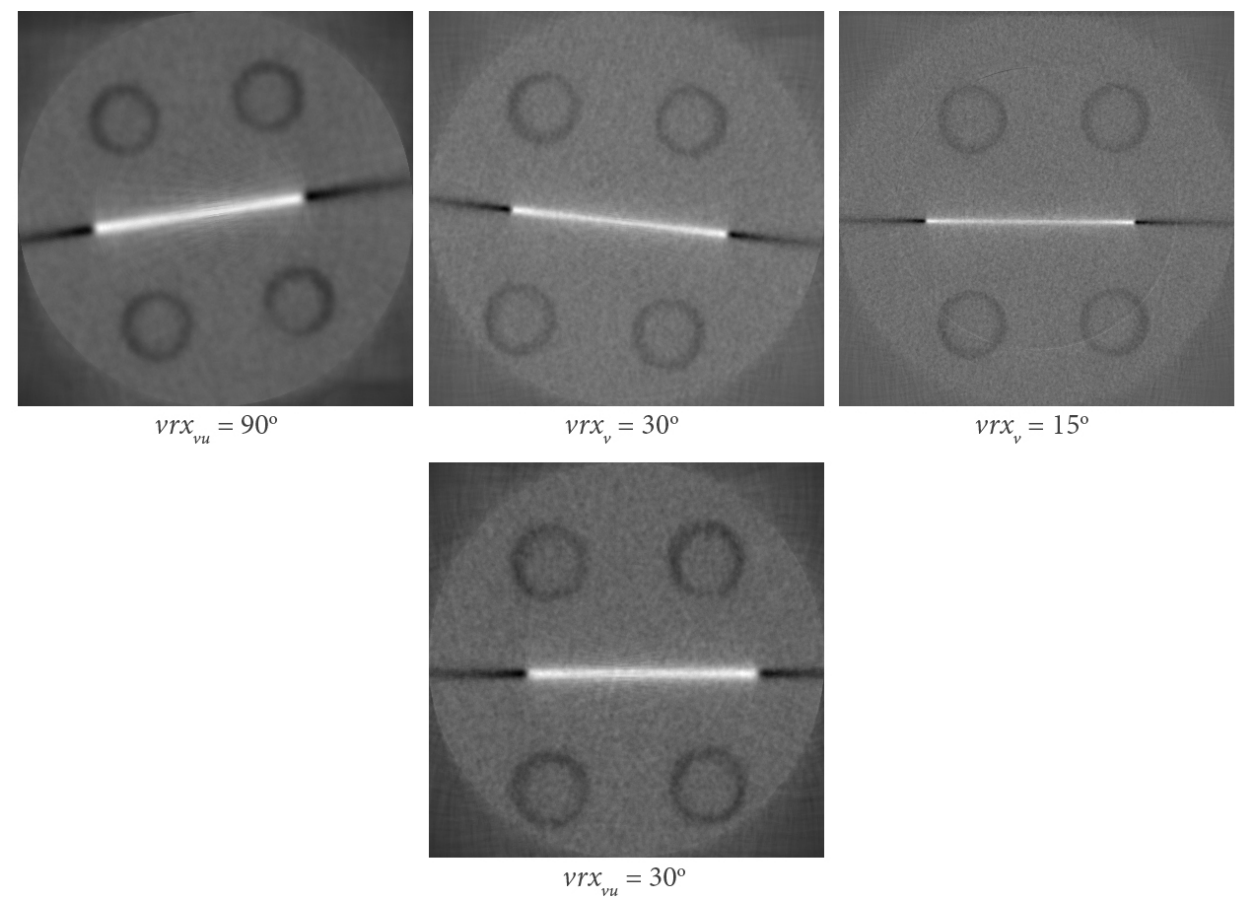

$v r x_{v}=15^{\circ}$

Figure 4.7: VRX-CBCT images of an isotropic MTF phantom.

The top images show the resolution improvement as the $v r x_{v}$ angle decreases. The image at the bottom taken at the isotropic vr $x_{v u}$ angle of $30^{\circ}$ shows no significant spatial resolution improvement over the rest of the images. 


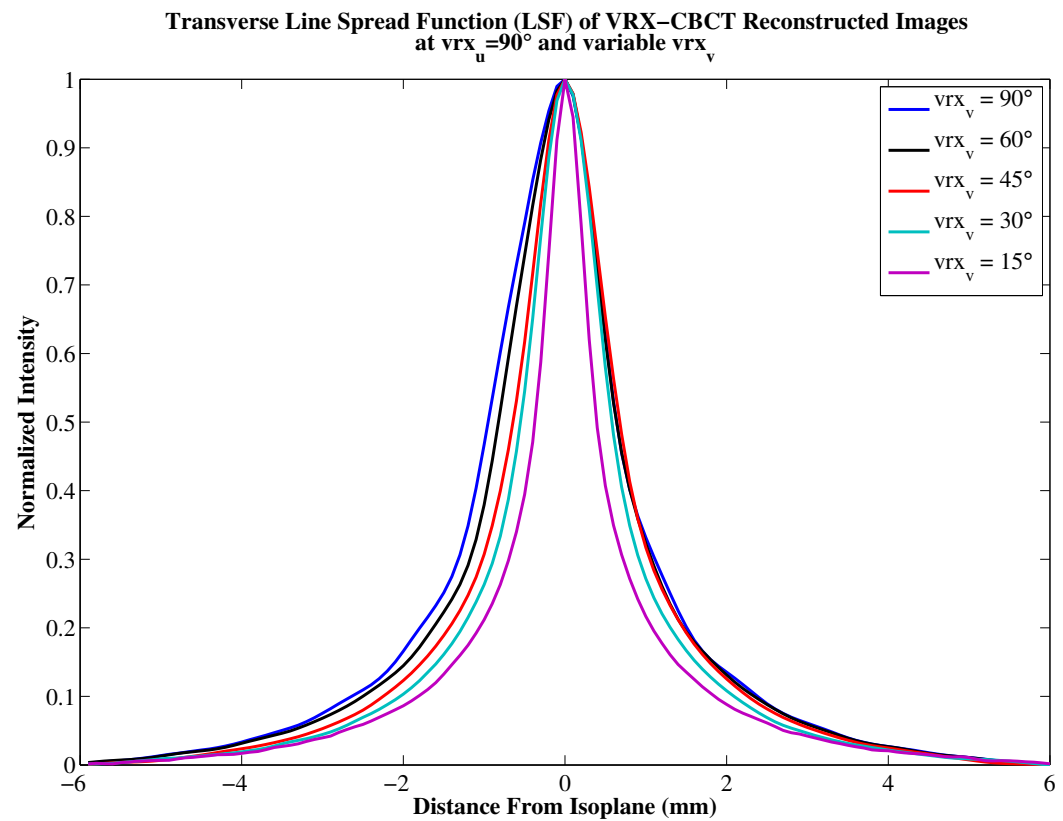

(a)

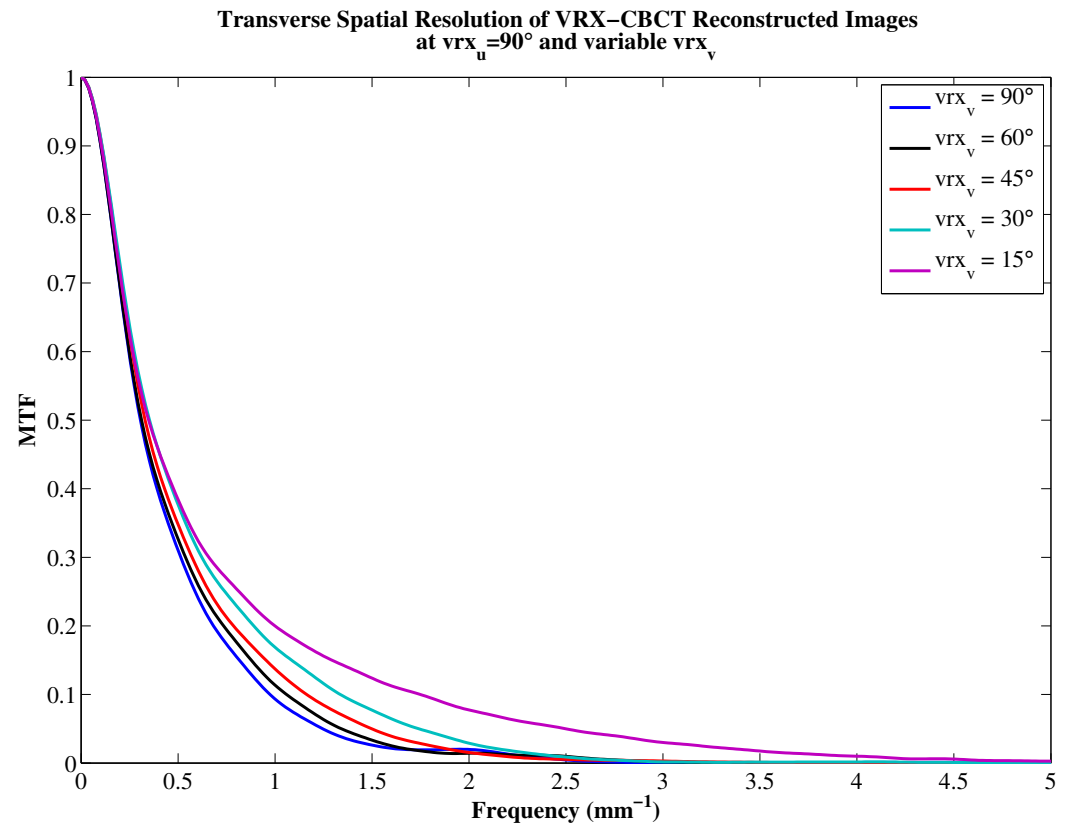

(b)

Figure 4.8: Transverse spatial resolution of the VRX-CBCT system at different $v r x_{v}$ angles using the 3D reconstruction algorithm.

(a) The line spread function and (b) the modulation transfer function. 


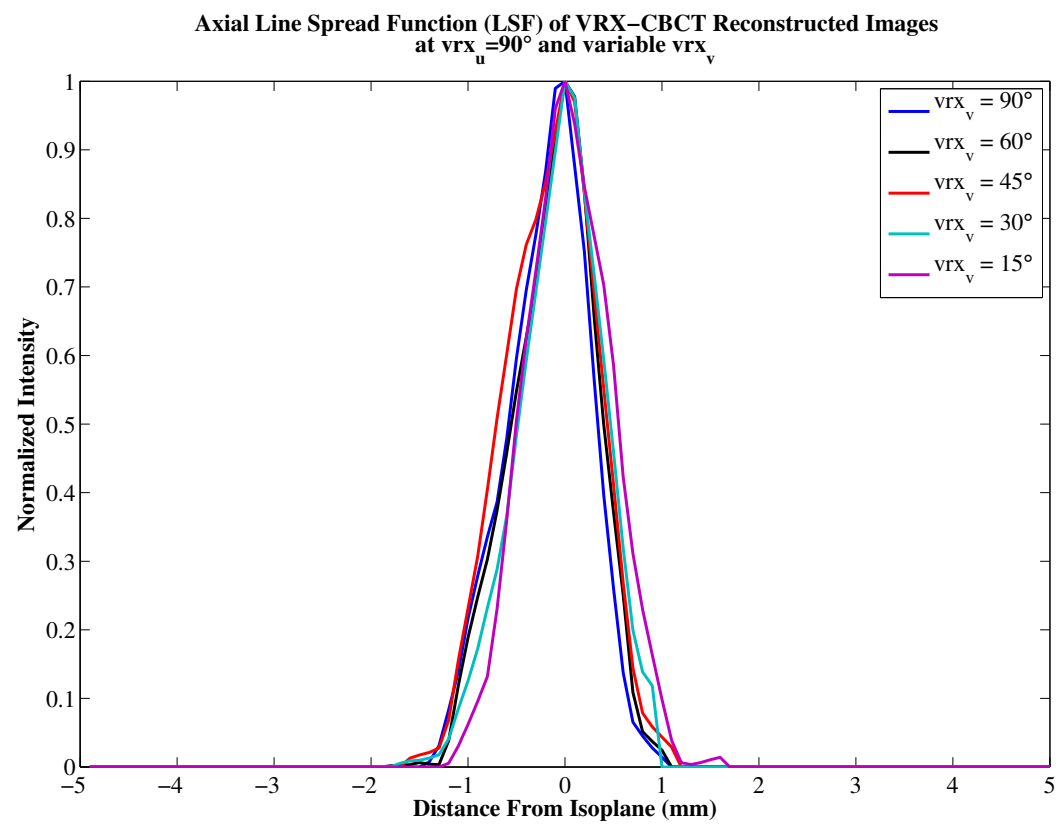

(a)

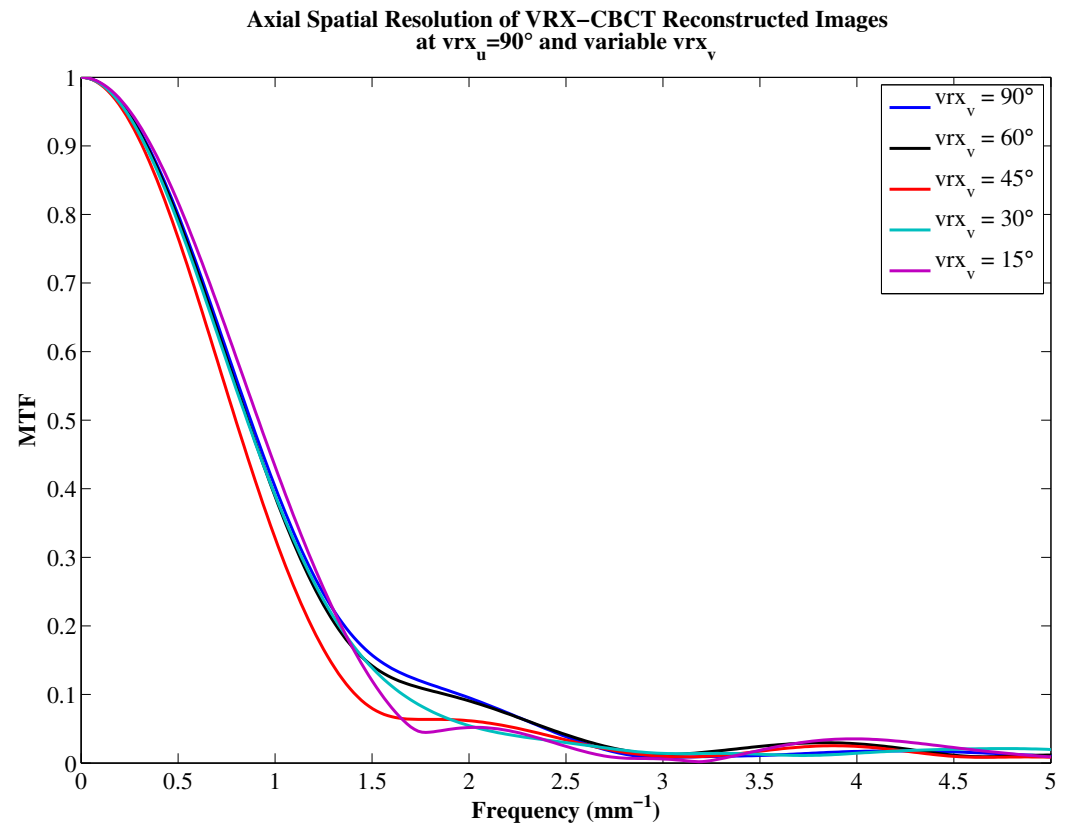

(b)

Figure 4.9: Axial spatial resolution of the VRX-CBCT system at different $v r x_{v}$ angles using the $3 \mathrm{D}$ reconstruction algorithm.

(a) The line spread function and (b) the modulation transfer function. 
to limited background material (acrylic) around the horizontal copper foil in the MTF phantom. The under-sampling could cause aliasing, which falsely improves the MTF curves at frequencies below the Nyquist frequency. This results in less accurate measurement of the spatial resolution.

Figure 4.10 illustrates the improvement in the axial spatial resolution of the 3D CT images as the $v r x_{u}$ angle is decreased. The result is comparable to the MTF curves in Figure 4.5, where the MTF increases for smaller vr $x_{u}$ angles. The overall MTF gain in Figure 4.10 compared to Figure 4.5 may be associated with the new 3D reconstruction algorithm used in the third phase of the study. The MTF curve at vr $x_{u}=60^{\circ}$ is slightly below the MTF curve at vr $x_{u}=90^{\circ}$. In a perfect system where there is no FPD cell cross-talk, the spatial resolution should improve $\sin 90^{\circ} / \sin 60^{\circ}=1.15$ or $15 \%$ over the spatial resolution of a comparable CBCT. In the current VRX-CBCT system, at $\operatorname{vr} x_{u}$ angles close to $60^{\circ}$, it is possible that the negative effect of signal smearing on the overall system MTF - described in Section 2.2.3.2 - is equal to or even more significant than any improvement in the spatial resolution of the system at such angles. In other words, there may be a range of $\operatorname{vr} x_{u}$ angles in the vicinity of $60^{\circ}$ that the overall spatial resolution of the VRX-CBCT system either does not improve at all or decreases compared to the MTF of a conventional CBCT.

The transverse spatial resolution of the VRX-CBCT system does not change significantly when the $v r x_{u}$ angle decreases (Figure 4.11), which - combined with the results from Figure 4.9 - supports the idea that the VRX effect only affects the resolution in the direction corresponding to the rotated FPD axis.

We were unable to detect a well-defined trend in the transverse spatial resolution of the VRX-CBCT system at different isotropic $v r x_{v u}$ angles down to $30^{\circ}$ (Figure 4.12). In general the transverse MTF curves for $v r x_{v u}$ of $90^{\circ}, 60^{\circ}$, and $45^{\circ}$ follow the same trend observed in Figures 4.2 and 4.8 with smaller MTF gains. The transverse MTF curve for $v r x_{v u}$ angle of $30^{\circ}$ is almost matching the corresponding curve for $45^{\circ}$. On the other hand, comparison of Figures 4.13 and 4.10 shows a slight increase in the improvement rate of the axial spatial resolution in isotropic VRX-CBCT system versus the resolution in the VRX-CBCT system, where the FPD is only tilted about its $u$-axis. It is important to point out that the $15^{\circ}$ curves are missing from the result of the isotropic experiment due to technical difficulties encountered when reconstructing the VRX-CBCT image of the MTF phantom at such a small isotropic angle. It is unknown if there would be any spatial resolution improvement in the transverse direction at $v r x_{v u}$ angles smaller than $30^{\circ}$. Nevertheless, lack of a significant improvement in the transverse spatial resolution of the isotropic VRX-CBCT was unexpected considering the results in the previous sections.

A few factors may have been involved in creating this phenomenon. Among those, inaccurate $3 \mathrm{D}$ calibration is believed to be the most likely reason for this outcome. In our experiments at $45^{\circ}$ and $30^{\circ}$, the automatic calibration process failed to find the most accurate horizontal offset value. A manual process to find the optimal offset values for CT images taken at $v r x_{u}=45^{\circ}$ and $30^{\circ}$, provided offset values that were up to $0.6 \mathrm{~mm}$ different from the automatic process results. This is most 


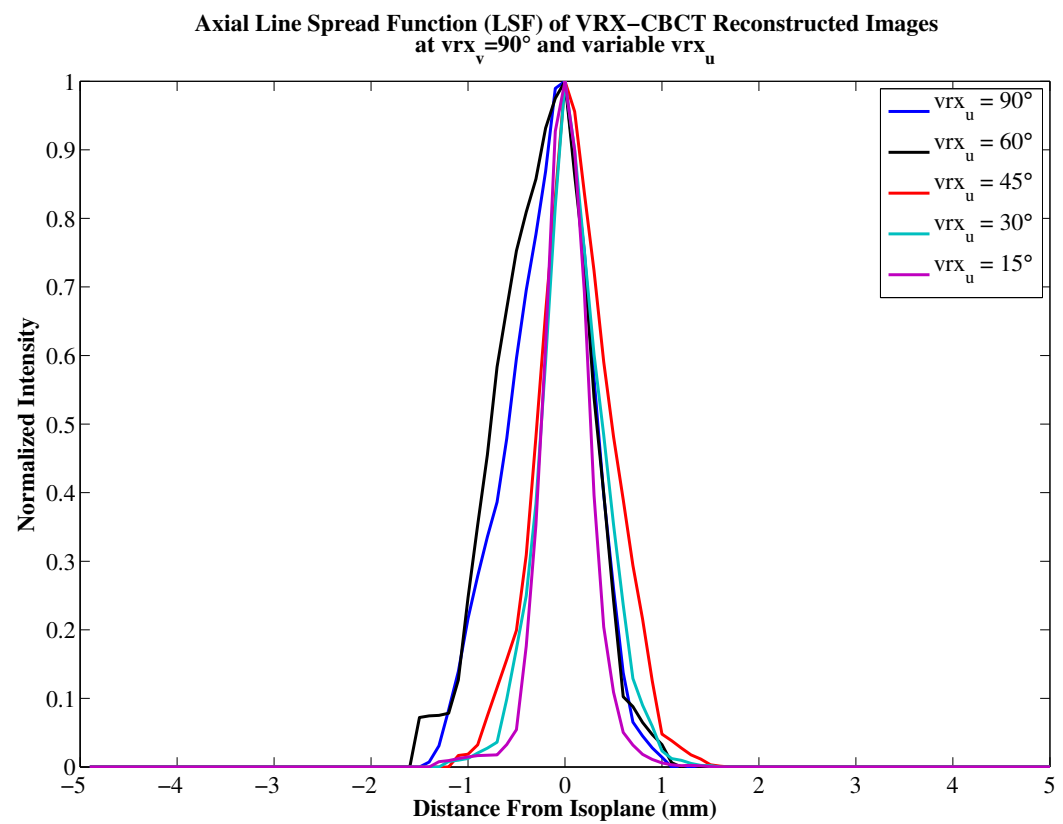

(a)

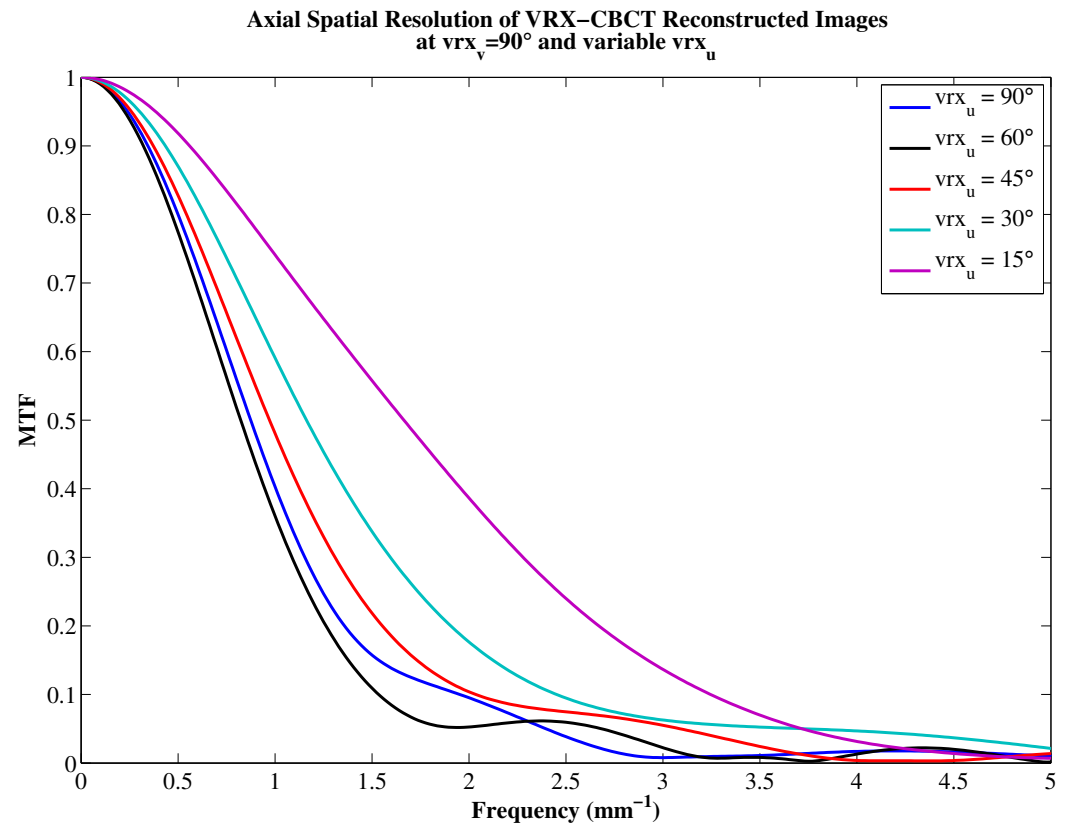

(b)

Figure 4.10: Axial spatial resolution of the VRX-CBCT system at different $v r x_{u}$ angles using the $3 \mathrm{D}$ reconstruction algorithm.

(a) The line spread function and (b) the modulation transfer function. 


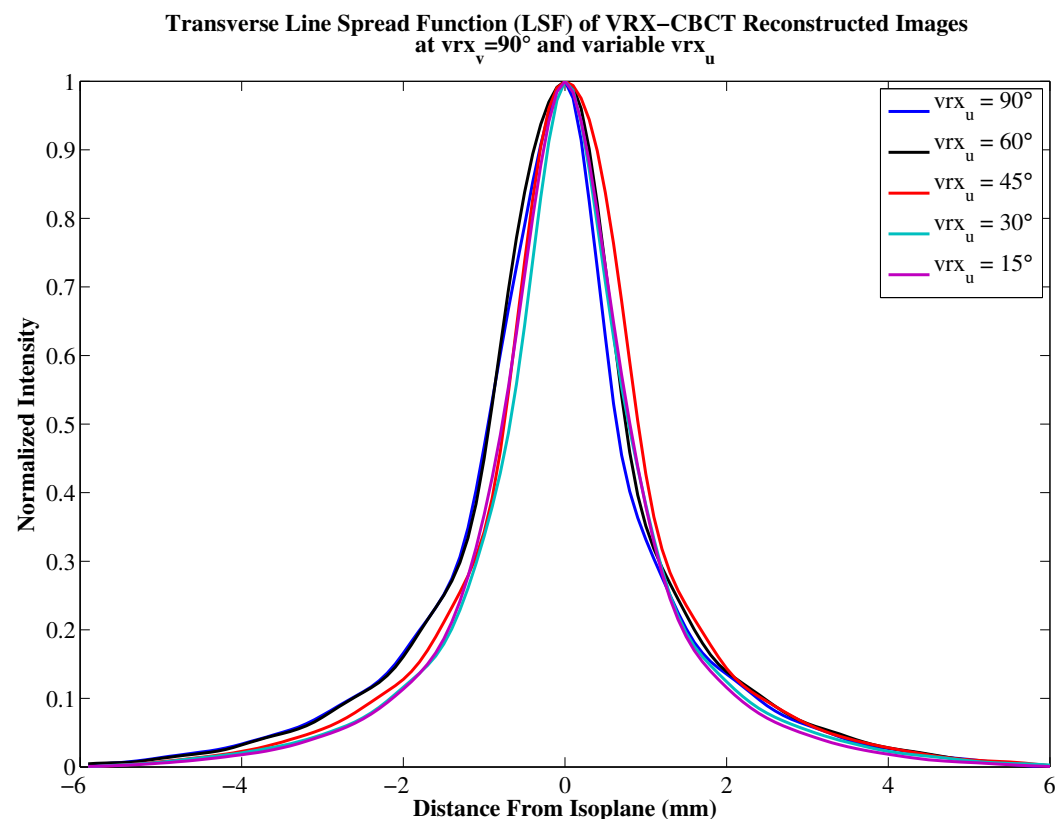

(a)

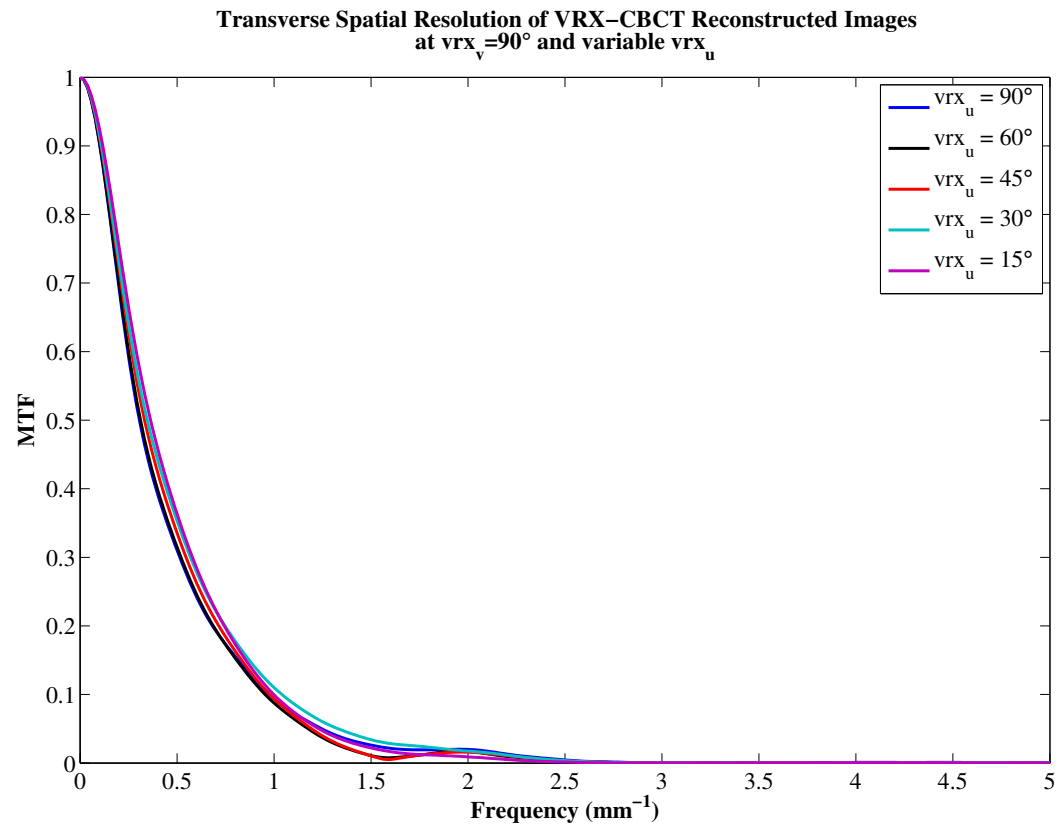

(b)

Figure 4.11: Transverse spatial resolution of the VRX-CBCT system at different $v r x_{u}$ angles.

(a) The line spread function and (b) the modulation transfer function. 


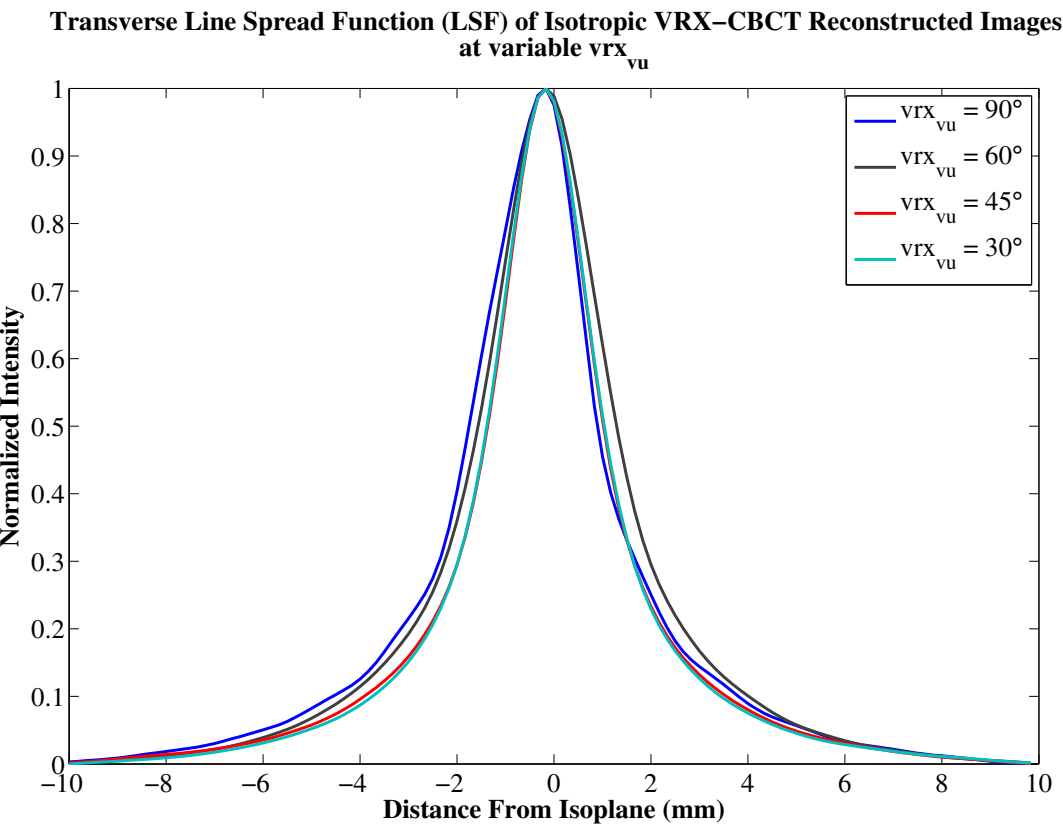

(a)

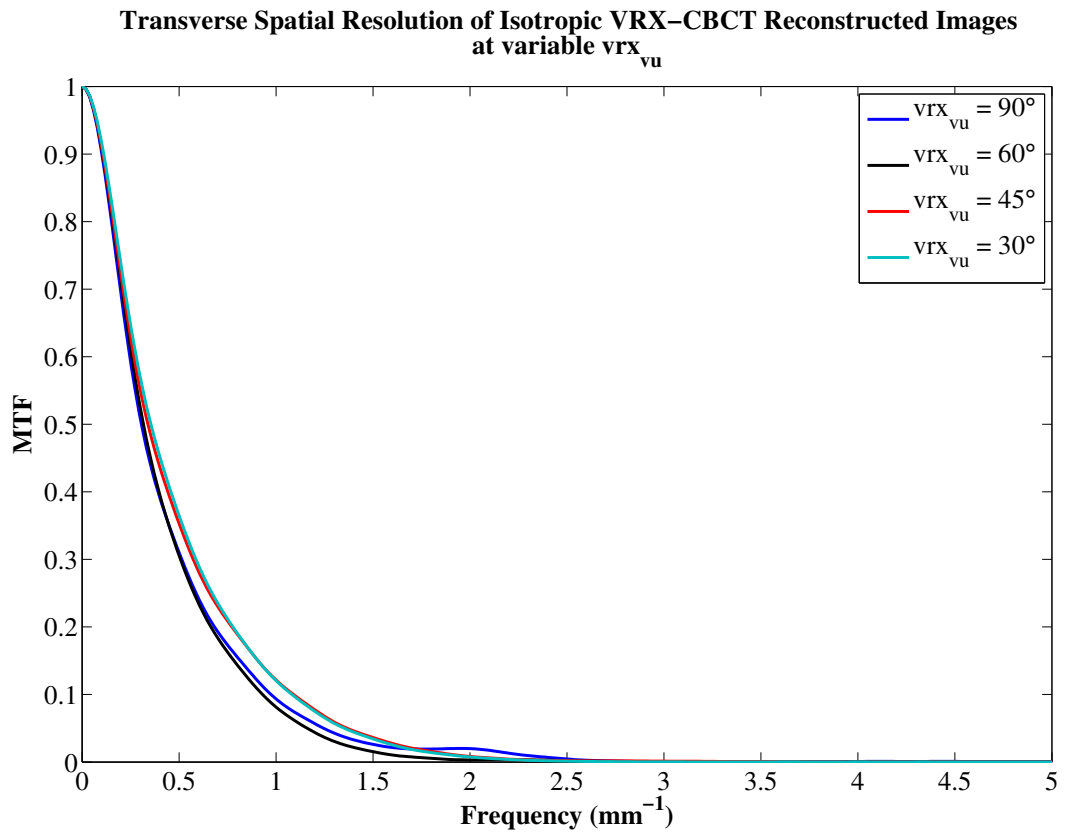

(b)

Figure 4.12: Transverse spatial resolution of the isotropic VRX-CBCT system at different vr $x_{v u}$ angles.

(a) The line spread function and (b) the modulation transfer function. 


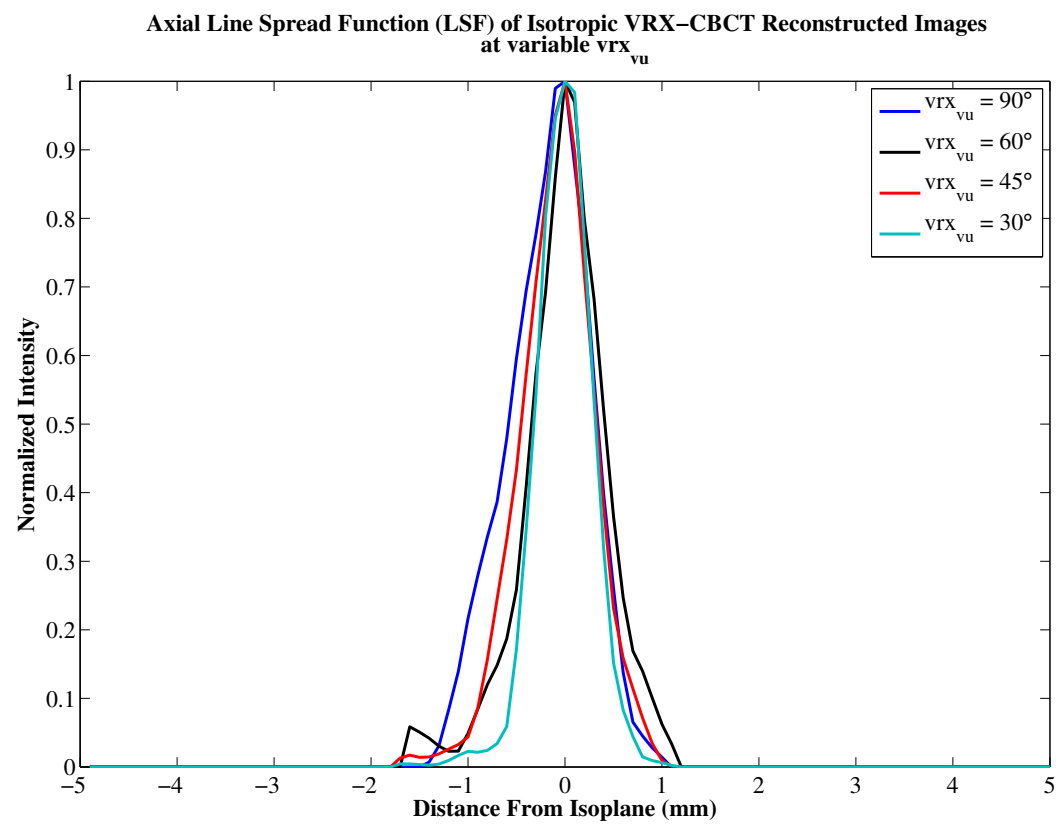

(a)

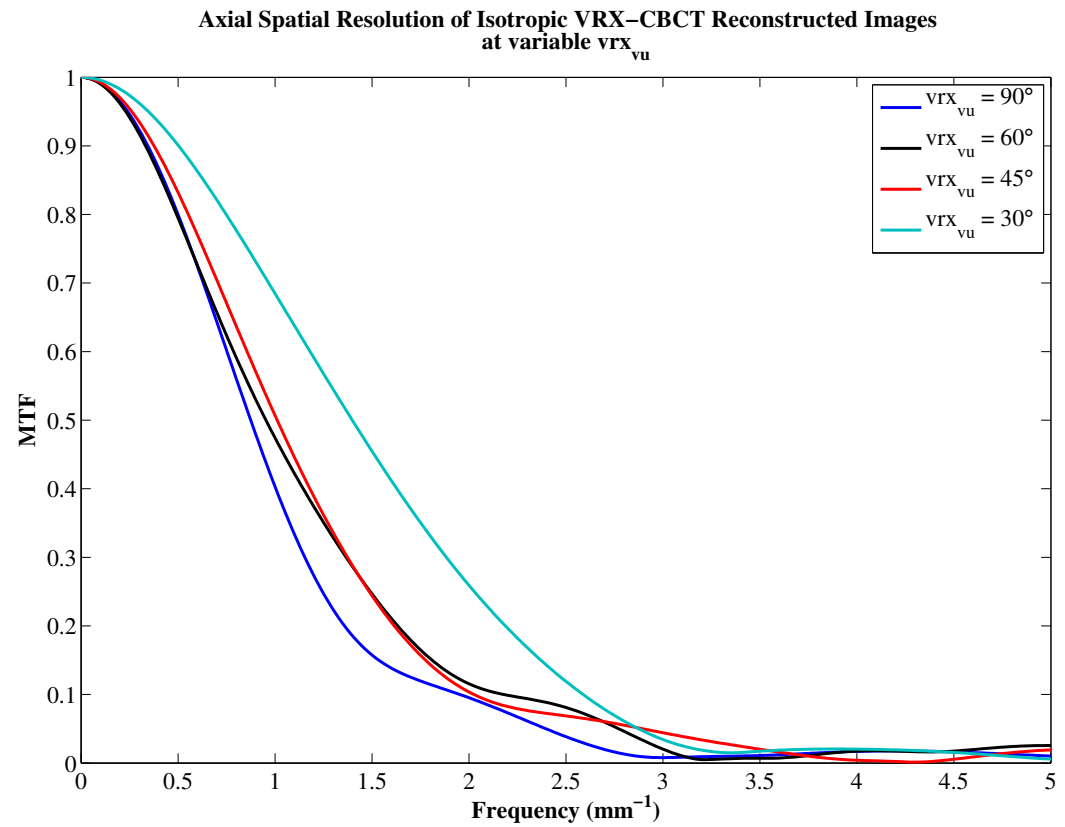

(b)

Figure 4.13: Axial spatial resolution of the isotropic VRX-CBCT system at different $v r x_{v u}$ angles.

(a) The line spread function and (b) the modulation transfer function. 
likely due to increased sensitivity of the system at small vr $x_{v u}$ angles to the slightest lateral movement of the MTF phantom. To overcome this problem, a highly precise calibration phantom needs to be manufactured that restricts the unwanted lateral movement of the steel balls during the scan to a fraction of the current precision.

Under-sampling of the data and more aliasing in the larger $v r x_{v u}$ angles compared to smaller angles could also have had an effect on the current behavior of the isotropic VRX-CBCT in the transverse direction. Although it seems less likely, the significant increase in the noise level at isotropic $v r x_{v u}$ angles, compared to that of corresponding non-isotropic $v r x_{v}$ and $v r x_{u}$ angles, may have also interfered with the calibration, reconstruction, and/or MTF measurement. A more comprehensive study of the isotropic VRX-CBCT is necessary to fully understand this lack of significant transverse resolution improvement in an isotropic VRX-CBCT. 


\section{Chapter 5}

\section{Conclusions}

In this study, we designed and evaluated a specific VRX-CBCT system that uses a micro-focal x-ray tube and a CsI-based indirect FPD. The spatial resolution of the system was studied at different vr $x_{v}$ and $v r x_{u}$ angles, where the FPD was tilted about its vertical $v$-axis and horizontal $u$-axis, respectively. In addition, the spatial resolution of an isotropic VRX-CBCT was measured and the result was compared to the non-isotropic cases.

Preliminary results for selected vr $x_{v}$ angles show that the overall transverse spatial resolution of the VRX-CBCT increases at small $v r x_{v}$ angles compared to a conventional CBCT. However, this does not imply that such observation is valid for all vr $x_{v}$ angles in the $90^{\circ}$ to $0^{\circ}$ range. In other words, there may be a range of $v r x_{v}$ angles between $90^{\circ}$ and $0^{\circ}$ for which the overall system MTF is equal to or even lower than that of a conventional CBCT.

Also it was shown in this study that, compared to a similar conventional CBCT, the axial spatial resolution of the VRX-CBCT system increased when the vr $x_{u}$ angle was set to small values. The rate of increase in the axial spatial resolution seems to be more than that of the transverse spatial resolution. The thinner CT slices created by such a device at small $v r x_{u}$ angles may be useful in detecting smaller lesions with low contrast that are otherwise not detectable using a conventional CBCT.

The isotropic VRX-CBCT did not deliver a significant improvement in spatial resolution in the transverse direction within the range of $v r x_{v u}$ angles we were able to operate. Comparing the MTF curves of the isotropic and non-isotropic settings reveals a measurable drop in overall efficiency of the VRX-CBCT when the FPD is tilted about both its axes.

\section{$5.1 \quad$ Limitations}

The limitations of this project can be summarized in the following statements:

1. The most important limitation of the VRX-CBCT system came from the detection of incident x-ray photons when the CsI-based FPD is tilted. At oblique detector angles, the trajectory of the x-ray photons within the CsI scintillator is on average longer than the thickness of the scintillator, therefore a single x-ray photon may pass over several detector cells, generating multiple light photons 
along the path. In addition, regardless of the x-ray incidence angle, the light photons generated in the CsI scintillator by interaction of one x-ray photon with the scintillator material, travel isotropically and may be absorbed by more than one detector cell. Although each individual detector cell in the FPD acts independently from other cells to provide a unique response to the amount of light absorbed, the combination of the above effects could cause smearing in the signal, which would in turn reduce the overall spatial resolution of the FPD.

2. Another important technical limitation of the current VRX-CBCT was our inability to acquire acceptable 3D CT images for isotropic $v r x_{v u}$ angles less than $30^{\circ}$, due to significant decrease in signal density in parts of the FPD where the frame and the protective material enclosing the detector blocked the x-ray photons before they reached the scintillator. In other words, the x-ray shadow of the FPD protective frame on the imaging plane created highly insensitive regions in the final image that prevented the calibration program from functioning correctly. Our attempt to increase the overall signal density by decreasing the source to detector distance (SDD) was also ineffective due to significant over-saturation of the FPD in near side caused by the increase in signal inhomogeneity. To overcome this problem, we suggest that the manufacturers of the indirect flat panel detectors place the scintillator as close to the protective surface as possible and use a more x-ray transparent material for the enclosing frame of the FPD.

3. Although the overall low performance of the current VRX-CBCT system in transverse MTF could be partially explained by the signal smearing effect described in the first section, the difference between the performance of the system in transverse versus the axial direction may suggest a limitation of the current 3D reconstruction algorithm. Even though the current version of VRX$\mathrm{CBCT}$ reconstruction algorithm has improved the overall MTF of the system in the transverse direction, multiple assumptions were made to numerically find the best intensity values for the projection images. More research should be done to increase the efficiency of this algorithm, including but not limited to more thoroughly studying the behavior of the FPD at oblique angles, and using the results to adjust the reconstruction algorithm's intensity calculation before back-projection.

4. A highly precise 3D calibration program is essential for the success of the VRX$\mathrm{CBCT}$ reconstruction algorithm. The current VRX-CBCT calibration program lacked precision in finding the exact position of the ball bearings on the projection images. Using the current method, the positions of the balls were measured using adaptive thresholding and center of intensity calculations. In reality, the center of intensity of the x-ray shadows of the calibration phantom ball bearings may not represent the actual position of the shadow of the ball bearings' center due to several factors, including the signal profile of such shadows on the FPD imaging plane. A more thorough study of the signal profile, as mentioned in the previous section, could lead to improvement in extracting the actual positions of 
the balls in the projection image and consequently a more accurate calibration program.

\subsection{Potential Applications}

A VRX-CBCT system affords unique advantages for several applications such as small animal imaging and digital mammography. In small animal imaging, the resolution of the $\mathrm{CT}$ images should always be maximized based on the size of the animal being scanned. This is the main advantage of the VRX-CBCT system over other high resolution CBCT devices such as micro-CT's. In digital mammography there is typically a need to examine a limited field of view at high resolution to determine structure of micro-calcifications or small soft tissue lesions. In current digital mammography units, a FPD is used to acquire a full field of view image and then a separate smaller FOV higher resolution detector is required to obtain the detail. A VRX-CBCT system could be used to acquire both types of image using the same equipment. In addition, it may be possible to combine the two phases into a single CT scan, by implementing a target imaging technique [59] in the current VRX-CBCT, and thus lowering the patient dose during a full CT scan.

It is important to realize that the 3D VRX technique is not limited to the current version of the VRX-CBCT system. Although the spatial resolution of the current VRX-CBCT is limited mainly by the characteristics of the FPD, the 3D VRX technique could potentially be used with almost any $2 \mathrm{D}$ x-ray detector, resulting in a possible increase in the spatial resolution of such CT device. As an example, tilting the detector of a conventional micro-CT could increase its spatial resolution, providing new opportunities in different applications. 


\section{List of References}

[1] J. Hsieh, Computed Tomography. Principles, Design, Artifacts, and Recent Advances. Bellingham, WA, SPIE Publications, 2003.

[2] J. T. Bushberg, J. A. Seibert, E. M. L. Jr., and J. M. Boone, The Essential Physics of Medical Imaging, 2nd ed. Philadelphia, PA, Lippincott Williams \& Wilkins, 2001.

[3] S. Achenbach, D. Ropers, A. Kuettner, T. Flohr, B. Ohnesorge, H. Bruder, H. Theessen, M. Karakaya, W. G. Daniel, W. Bautz, W. A. Kalender, and K. Anders, "Contrast-enhanced coronary artery visualization by dual-source computed tomography-initial experience," Eur J Radiol, vol. 57, no. 3, pp. 331-335, 2006.

[4] E. Samei, N. T. Ranger, J. T. Dobbins, and Y. Chen, "Intercomparison of methods for image quality characterization. I. Modulation transfer function," Med Phys, vol. 33, no. 5, pp. 1454-1465, 2006.

[5] X-ray. [Online]. Available: http://student.britannica.com/elementary/art-66143 [Accessed: 4/12/2009].

[6] W. Zhao, G. Ristic, and J. A. Rowlands, "X-ray imaging performance of structured cesium iodide scintillators," Med Phys, vol. 31, no. 9, pp. 2594-2605, 2004.

[7] R. Ning, X. Tang, and D. Conover, "X-ray scatter correction algorithm for cone beam CT imaging," Med Phys, vol. 31, no. 5, pp. 1195-1202, 2004.

[8] S. J. Boyce and E. Samei, "Imaging properties of digital magnification radiography," Med Phys, vol. 33, no. 4, pp. 984-996, 2006.

[9] J. E. Gray and M. Trefler, "Phase effects in diagnostic radiological images," Med Phys, vol. 3, no. 4, pp. 195-203, 1976.

[10] M. R. Ay, S. Sarkar, M. Shahriari, D. Sardari, and H. Zaidi, "Assessment of different computational models for generation of x-ray spectra in diagnostic radiology and mammography," Med Phys, vol. 32, no. 6, pp. 1660-1675, 2005.

[11] I. M. Gagne and D. M. Robinson, "The impact of tumor motion upon CT image integrity and target delineation," Med Phys, vol. 31, no. 12, pp. 3378-3392, 2004.

[12] P. J. La Riviere and P. Vargas, "Correction for resolution nonuniformities caused by anode angulation in computed tomography." IEEE Trans Med Imaging, vol. 27 , no. 9, pp. 1333-1341, 2008. 
[13] J. M. Boone, "Determination of the presampled MTF in computed tomography," Med Phys, vol. 28, no. 3, pp. 356-360, 2001.

[14] S. Greschus, F. Kiessling, M. P. Lichy, J. Moll, M. M. Mueller, R. Savai, F. Rose, C. Ruppert, A. Gunther, M. Luecke, N. E. Fusenig, W. Semmler, and H. Traupe, "Potential applications of flat-panel volumetric CT in morphologic and functional small animal imaging," Neoplasia, vol. 7, no. 8, pp. 730-740, 2005.

[15] C. Nimsky, O. Ganslandt, P. Hastreiter, R. Wang, T. Benner, A. G. Sorensen, and R. Fahlbusch, "Preoperative and intraoperative diffusion tensor imagingbased fiber tracking in glioma surgery," Neurosurgery, vol. 56, no. 1, pp. 130-137, 2005.

[16] A. Gopal and S. S. Samant, "Bar-pattern technique for modulation transfer function measurement in portal imaging," Proceedings of SPIE - The International Society for Optical Engineering, vol. 5030 I, pp. 433-444, 2003.

[17] B. Nielsen, "Measurement of the focal spot size of diagnostic x-ray tubes by the pinhole camera technique. the influence of the pinhole on the measured focal spot size." Health Phys, vol. 38, no. 4, pp. 683-686, 1980.

[18] J. Law, "Measurement of focal spot size in mammography x-ray tubes." $\mathrm{Br} J$ Radiol, vol. 66, no. 781, pp. 44-50, 1993.

[19] S. Mori, M. Endo, K. Nishizawa, M. Ohno, H. Miyazaki, K. Tsujita, and Y. Saito, "Prototype heel effect compensation filter for cone-beam ct." Phys Med Biol, vol. 50, no. 22, pp. N359-70, 2005.

[20] J. R. Vetter and J. E. Holden, "Correction for scattered radiation and other background signals in dual-energy computed tomography material thickness measurements." Med Phys, vol. 15, no. 5, pp. 726-731, 1988.

[21] J. T. Dobbins, D. L. Ergun, L. Rutz, D. A. Hinshaw, H. Blume, and D. C. Clark, "DQE(f) of four generations of computed radiography acquisition devices," Med Phys, vol. 22, no. 10, pp. 1581-1593, 1995.

[22] M. Albert and A. D. Maidment, "Linear response theory for detectors consisting of discrete arrays." Med Phys, vol. 27, no. 10, pp. 2417-2434, 2000.

[23] J. Morishita, K. Doi, R. Bollen, P. C. Bunch, D. Hoeschen, G. Sirand-rey, and Y. Sukenobu, "Comparison of two methods for accurate measurement of modulation transfer functions of screen-film systems," Med Phys, vol. 22, no. 2, pp. 193-200, 1995.

[24] A. Kuhls-Gilcrist, G. Yadava, V. Patel, A. Jain, D. Bednarek, and S. Rudin, "The solid-state x-ray image intensifier (ssxii): An emccd-based x-ray detector." SPIE - The International Society for Optical Engineering, vol. 6913, pp. 69130K-1 69 130K-10, 2008. 
[25] I. A. Cunningham and A. Fenster, "A method for modulation transfer function determination from edge profiles with correction for finite-element differentiation," Med Phys, vol. 14, no. 4, pp. 533-537, 1987.

[26] H. Fujita, K. Doi, and M. L. Giger, "Investigation of basic imaging properties in digital radiography. 6. MTFs of II-TV digital imaging systems," Med Phys, vol. 12 , no. 6 , pp. $713-720,1985$.

[27] A. Sasov, "Desktop x-ray micro-CT instruments," Proceedings of SPIE - The International Society for Optical Engineering, vol. 4503, pp. 282-290, 2001.

[28] R. Birch and M. Marshall, "Computation of bremsstrahlung x-ray spectra and comparison with spectra measured with a $\mathrm{Ge}(\mathrm{Li})$ detector," Phys Med Biol, vol. 24, no. 3, pp. 505-517, 1979.

[29] F. A. DiBianca, V. Gupta, and H. D. Zeman, "A variable resolution x-ray detector for computed tomography: I. Theoretical basis and experimental verification," Med Phys, vol. 27, no. 8, pp. 1865-1874, 2000.

[30] R. Melnyk and F. A. DiBianca, "Modeling and measurement of the detector presampling MTF of a variable resolution x-ray CT scanner," Med Phys, vol. 34, no. 3, pp. 1062-1075, 2007.

[31] S. C. Lee, H. K. Kim, I. K. Chun, M. H. Cho, S. Y. Lee, and M. H. Cho, "A flatpanel detector based micro-CT system: performance evaluation for small-animal imaging," Phys Med Biol, vol. 48, no. 24, pp. 4173-4185, 2003.

[32] G. Borasi, E. Samei, M. Bertolini, A. Nitrosi, and D. Tassoni, "Contrast-detail analysis of three flat panel detectors for digital radiography," Med Phys, vol. 33, no. 6, pp. 1707-1719, 2006.

[33] J. E. Tkaczyk, B. Claus, D. G. Trotter, and J. W. Eberhard, "Response of a $\mathrm{CsI} /$ amorphous-Si flat panel detector as function of incident x-ray angle," SPIE - The International Society for Optical Engineering, vol. 6142, pp. $61423 \mathrm{~F}-1$ $61423 \mathrm{~F}-10,2006$.

[34] S. G. Armato, D. J. Doshi, R. Engelmann, C. L. Croteau, and H. MacMahon, "Temporal subtraction in chest radiography: automated assessment of registration accuracy," Med Phys, vol. 33, no. 5, pp. 1239-1249, 2006.

[35] P. C. Johns and M. J. Yaffe, "Theoretical optimization of dual-energy x-ray imaging with application to mammography," Med Phys, vol. 12, no. 3, pp. 289$296,1985$.

[36] E. E. Graves, J. Ripoll, R. Weissleder, and V. Ntziachristos, "A submillimeter resolution fluorescence molecular imaging system for small animal imaging," Med Phys, vol. 30, no. 5, pp. 901-911, 2003. 
[37] B. S. Halpern, M. Dahlbom, A. Quon, C. Schiepers, C. Waldherr, D. H. Silverman, O. Ratib, and J. Czernin, "Impact of patient weight and emission scan duration on PET/CT image quality and lesion detectability," J Nucl Med, vol. 45, no. 5, pp. 797-801, 2004.

[38] W. A. Kalender, "X-ray computed tomography," Phys Med Biol, vol. 51, no. 13, pp. R29-43, 2006.

[39] T. G. Schmidt, J. Star-Lack, N. R. Bennett, S. R. Mazin, E. G. Solomon, R. Fahrig, and N. J. Pelc, "A prototype table-top inverse-geometry volumetric CT system," Med Phys, vol. 33, no. 6, pp. 1867-1878, 2006.

[40] N. L. Ford, M. M. Thornton, and D. W. Holdsworth, "Fundamental image quality limits for microcomputed tomography in small animals," Med Phys, vol. 30, no. 11, pp. 2869-2877, 2003.

[41] M. L. Giger and K. Doi, "Investigation of basic imaging properties in digital radiography. I. Modulation transfer function," Med Phys, vol. 11, no. 3, pp. 287295, 1984.

[42] W. M. Kohrt, "Preliminary evidence that DEXA provides an accurate assessment of body composition," J Appl Physiol, vol. 84, no. 1, pp. 372-377, 1998.

[43] E. L. Nickoloff and R. Riley, "A simplified approach for modulation transfer function determinations in computed tomography," Med Phys, vol. 12, no. 4, pp. 437-442, 1985.

[44] M. Ohkubo, S. Wada, T. Matsumoto, and K. Nishizawa, "An effective method to verify line and point spread functions measured in computed tomography," Med Phys, vol. 33, no. 8, pp. 2757-2764, 2006.

[45] I. A. Feldkamp, L. C. Davis, and J. W. Kress, "Practical cone-beam algorithm," Journal of the Optical Society of America A: Optics and Image Science, and Vision, vol. 1, no. 6, pp. 612-619, 1984.

[46] J. H. Siewerdsen, D. J. Moseley, B. Bakhtiar, S. Richard, and D. A. Jaffray, "The influence of antiscatter grids on soft-tissue detectability in cone-beam computed tomography with flat-panel detectors," Med Phys, vol. 31, no. 12, pp. 3506-3520, 2004 .

[47] R. Weissleder and U. Mahmood, "Molecular imaging." Radiology, vol. 219, no. 2, pp. 316-333, 2001.

[48] R. Weissleder and M. J. Pittet, "Imaging in the era of molecular oncology." Nature, vol. 452, no. 7187, pp. 580-589, 2008.

[49] D. W. Holdsworth and M. M. Thornton, "Micro-CT in small animal and specimen imaging," Trends in Biotechnology, vol. 20, no. 8, pp. S34-S39, 2002. 
[50] E. Ritman, "Molecular imaging in small animals-roles for micro-CT," J Cell Biochem Suppl, vol. 39, pp. 116-124, 2002.

[51] E. L. Ritman, "Micro-computed tomography-current status and developments," Annu Rev Biomed Eng, vol. 6, pp. 185-208, 2004.

[52] A. Sasov and D. Dewaele, "High-resolution in-vivo micro-CT scanner for small animals," Proceedings of SPIE - The International Society for Optical Engineering, vol. 4503, pp. 256-264, 2001.

[53] W. Haddad, I. McNulty, J. Trebes, E. Anderson, R. Levesque, and L. Yang, "Ultrahigh-resolution x-ray tomography," Science, vol. 266, no. 5188, pp. 1213$1215,1994$.

[54] M. J. Paulus, S. S. Gleason, S. J. Kennel, P. R. Hunsicker, and D. K. Johnson, "High resolution x-ray computed tomography: an emerging tool for small animal cancer research," Neoplasia, vol. 2, no. 1-2, pp. 62-70, 2000.

[55] F. A. DiBianca, P. Zou, L. M. Jordan, J. S. Laughter, H. D. Zeman, and J. Sebes, "A variable resolution x-ray detector for computed tomography: II. Imaging theory and performance," Med Phys, vol. 27, no. 8, pp. 1875-1880, 2000.

[56] F. A. DiBianca, R. Melnyk, A. Sambari, L. M. Jordan, J. S. Laughter, and P. Zou, "Solid-state VRX CT detector," SPIE - The International Society for Optical Engineering, vol. 3977, pp. 205-210, 2000.

[57] F. A. DiBianca, R. Melnyk, C. Duckworth, S. Russ, L. M. Jordan, and J. S. Laughter, "Comparison of VRX CT scanner geometries," SPIE - The International Society for Optical Engineering, vol. 4320, pp. 627-635, 2001.

[58] R. Melnyk, "Theoretical and experimental evaluation of spatial resolution in a variable resolution x-ray computed tomography scanner," Ph.D. dissertation, The University of Tennessee Health Science Center, December 2007.

[59] F. A. DiBianca, D. Gulabani, L. M. Jordan, S. Vangala, D. Rendon, J. S. Laughter, R. Melnyk, M. W. Gaber, and G. S. Keyes, "Four-arm variable-resolution x-ray detector for CT target imaging," SPIE - The International Society for Optical Engineering, vol. 5745, no. I, pp. 332-339, 2005.

[60] D. A. Rendon, F. A. DiBianca, and G. S. Keyes, "Comparison of multi-arm VRX CT scanners through computer models," SPIE - The International Society for Optical Engineering, vol. 6510, pp. $65103 \mathrm{Y}-1$ - $65103 \mathrm{Y}-9,2007$.

[61] M. W. Gaber, C. M. Wilson, C. D. Duntsch, H. Shukla, J. A. Zawaski, L. M. Jordan, D. A. Rendon, S. Vangala, G. S. Keyes, and F. A. DiBianca, "Volumetric analysis of tumors in rodents using the variable resolution x-ray (VRX) CTscanner," SPIE - The International Society for Optical Engineering, vol. 5746, pp. 9-15, 2005. 
[62] R. T. Droege and R. L. Morin, "A practical method to measure the MTF of CT scanners," Med Phys, vol. 9, no. 5, pp. 758-760, 1982.

[63] J. T. Dobbins, "Effects of undersampling on the proper interpretation of modulation transfer function, noise power spectra, and noise equivalent quanta of digital imaging systems," Med Phys, vol. 22, no. 2, pp. 171-181, 1995.

[64] B. Dahi, G. S. Keyes, D. A. Rendon, and F. A. DiBianca, "Performance analysis of a CsI-based flat panel detector in a cone beam variable resolution x-ray system," SPIE - The International Society for Optical Engineering, vol. 6510, pp. 65 104B-1 - 65 104B-8, 2007.

[65] B. Dahi, G. S. Keyes, D. A. Rendon, and F. A. Dibianca, "Analysis of axial spatial resolution in a variable resolution x-ray cone beam CT (VRX-CBCT) system," SPIE - The International Society for Optical Engineering, vol. 6913, pp. $69134 \mathrm{Y}-1-69134 \mathrm{Y}-8,2008$.

[66] Varian, "PaxScan ${ }^{\circledR}$ 2020," Accessed on: 03/23/2009. [Online]. Available: http://www.varian.com/media/xray/products/pdf/ps2020.pdf

[67] L. M. Jordan, F. A. DiBianca, R. Melnyk, A. Choudhary, H. Shukla, J. Laughter, and M. W. Gaber, "Determination of calibration parameters of a VRX CT system using an 'amoeba' algorithm," Journal of X-Ray Science and Technology, vol. 12, no. 4, pp. 281-293, 2004.

[68] L. A. Shepp and B. F. Logan Jr., "Fourier reconstruction of a head section," IEEE Transactions on Nuclear Science, vol. NS-21, no. 3, pp. 21-43, 1974.

[69] A. C. Kak and M. Slaney, Principles of Computerized Tomographic Imaging. New York, NY, USA: IEEE Press, 1988.

[70] J. C. Lagarias, J. A. Reeds, M. H. Wright, and P. E. Wright, "Convergence properties of the Nelder-Mead simplex method in low dimensions," SIAM Journal of Optimization, vol. 9, no. 1, pp. 112-147, 1998. 


\section{Appendix A}

\section{VRX-CBCT Calibration Code}

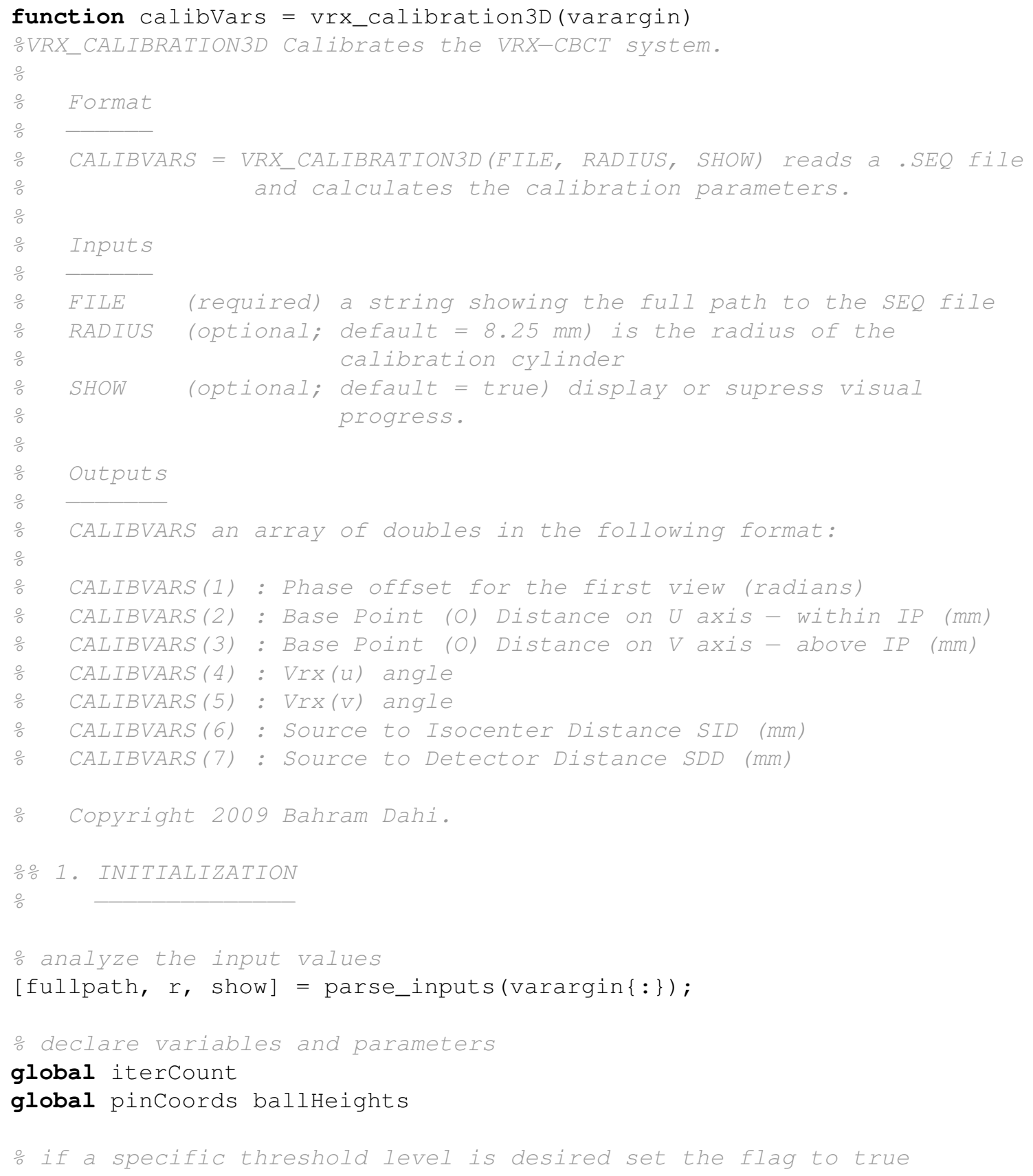




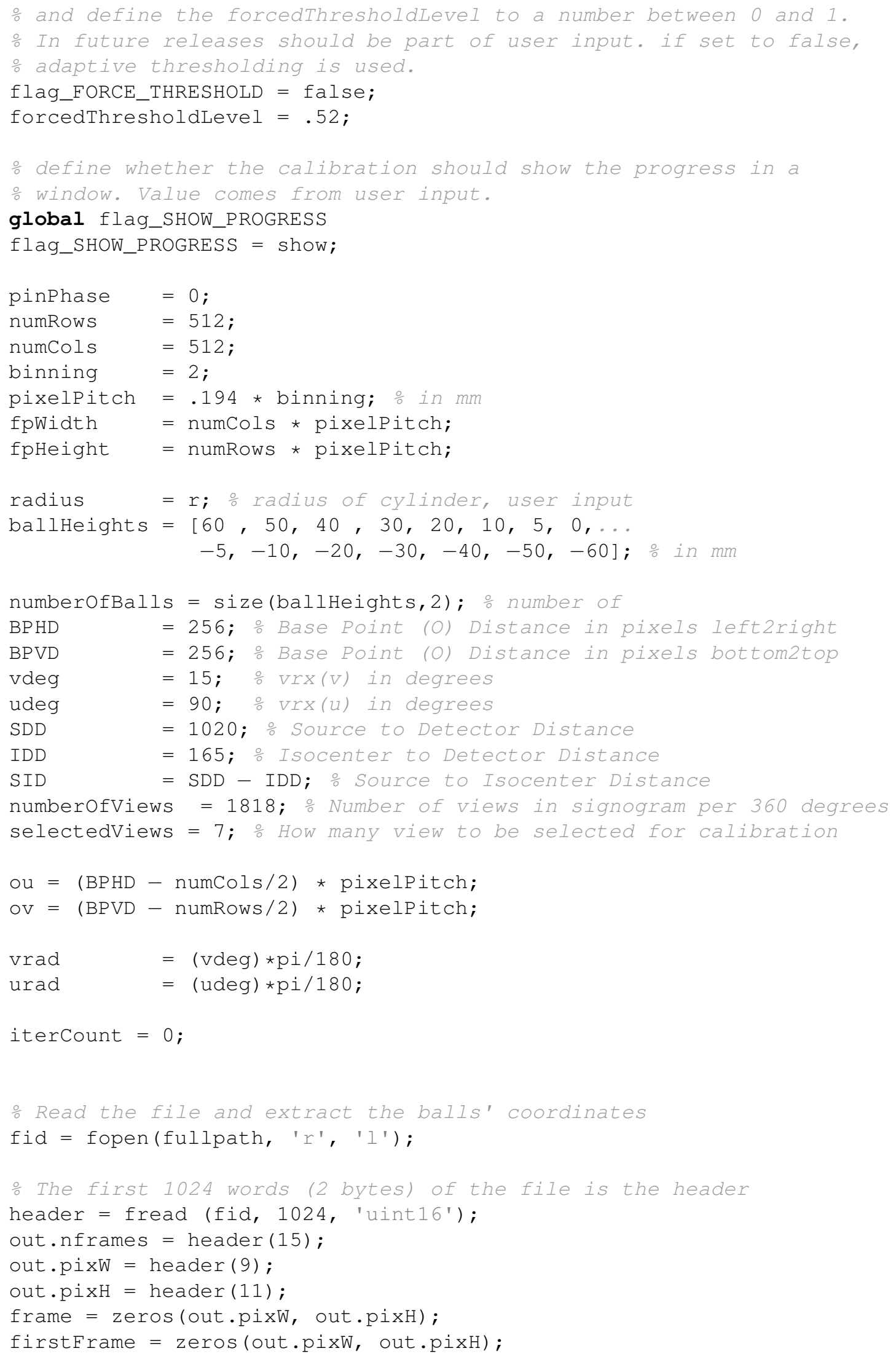




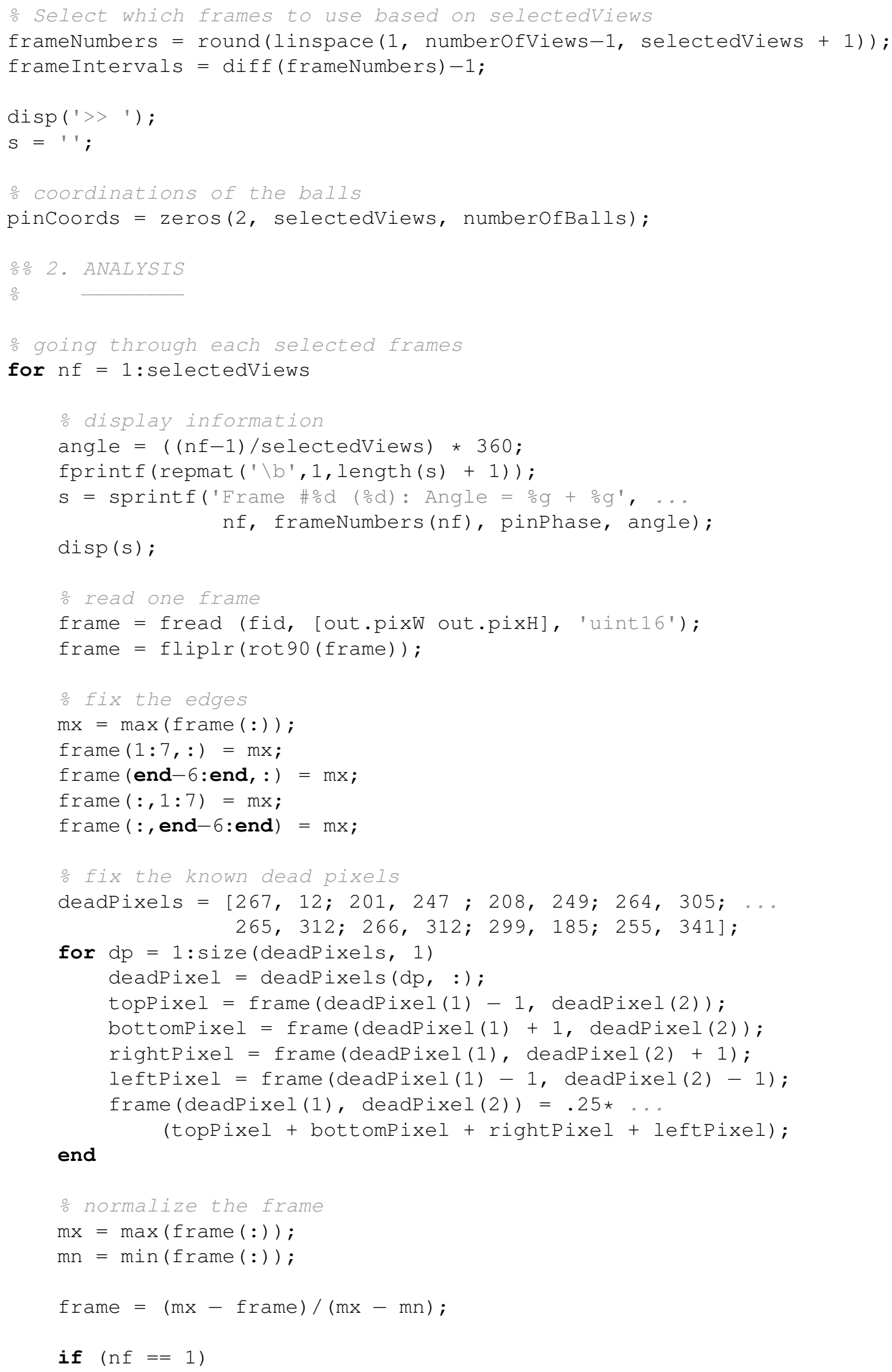




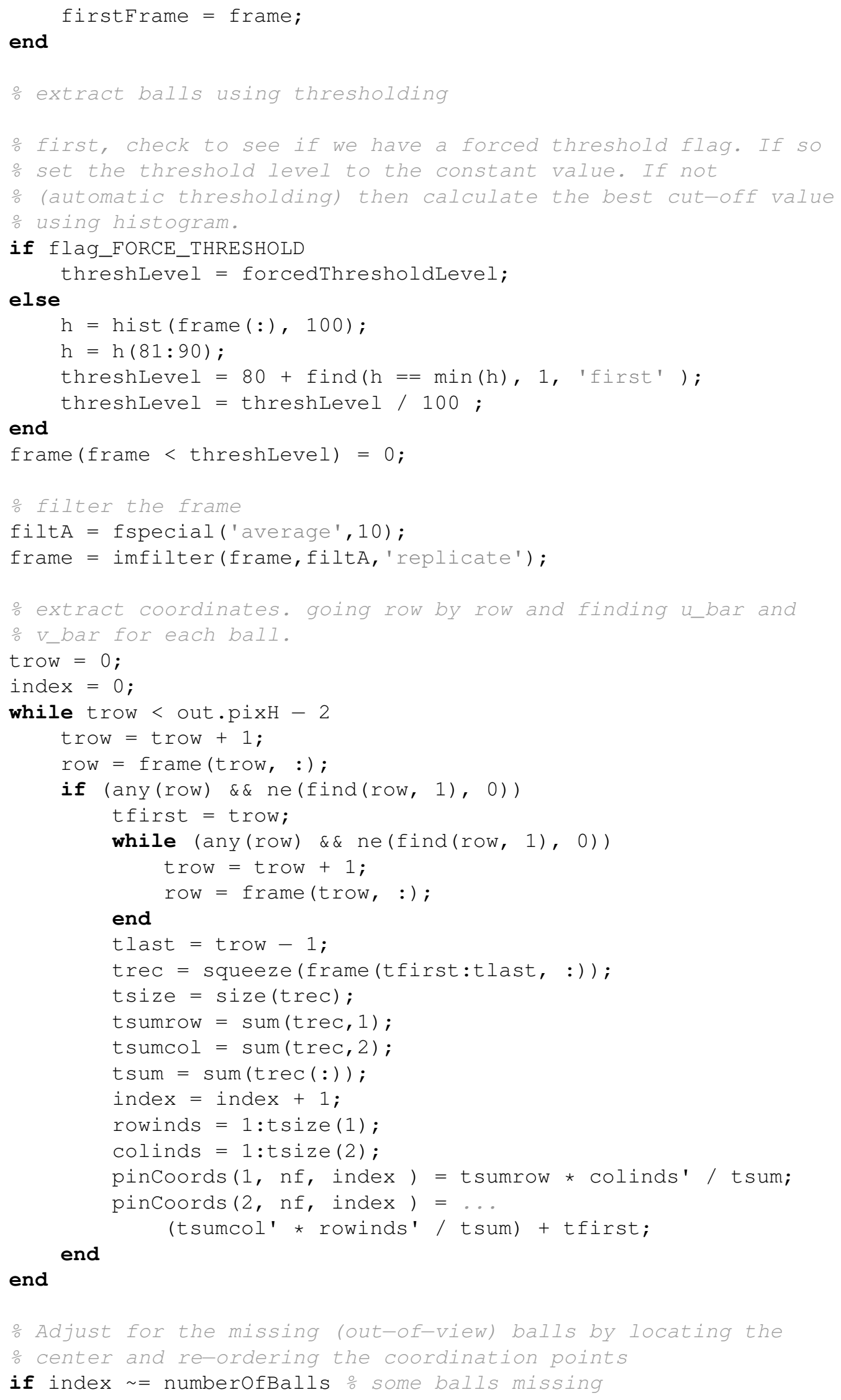




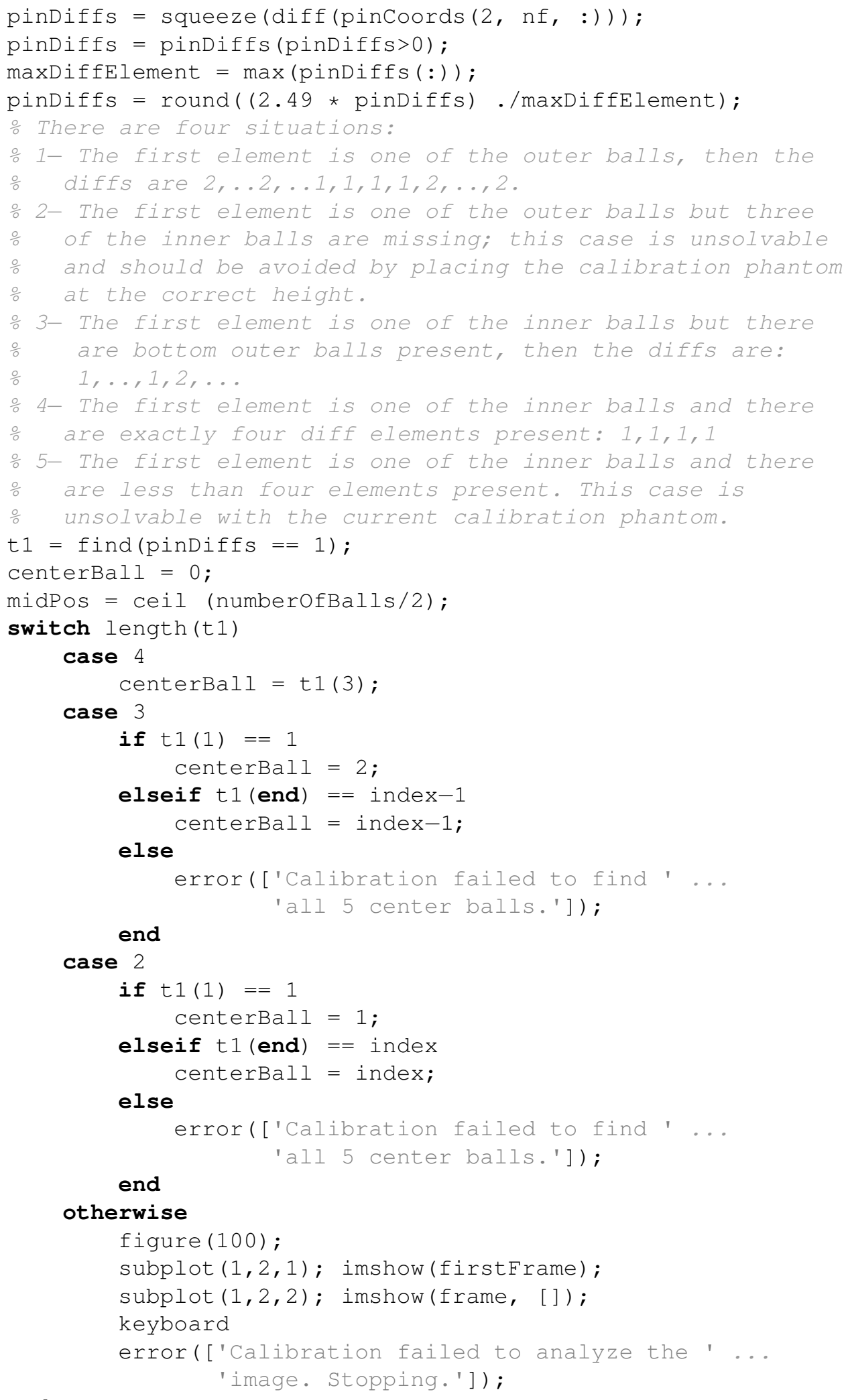




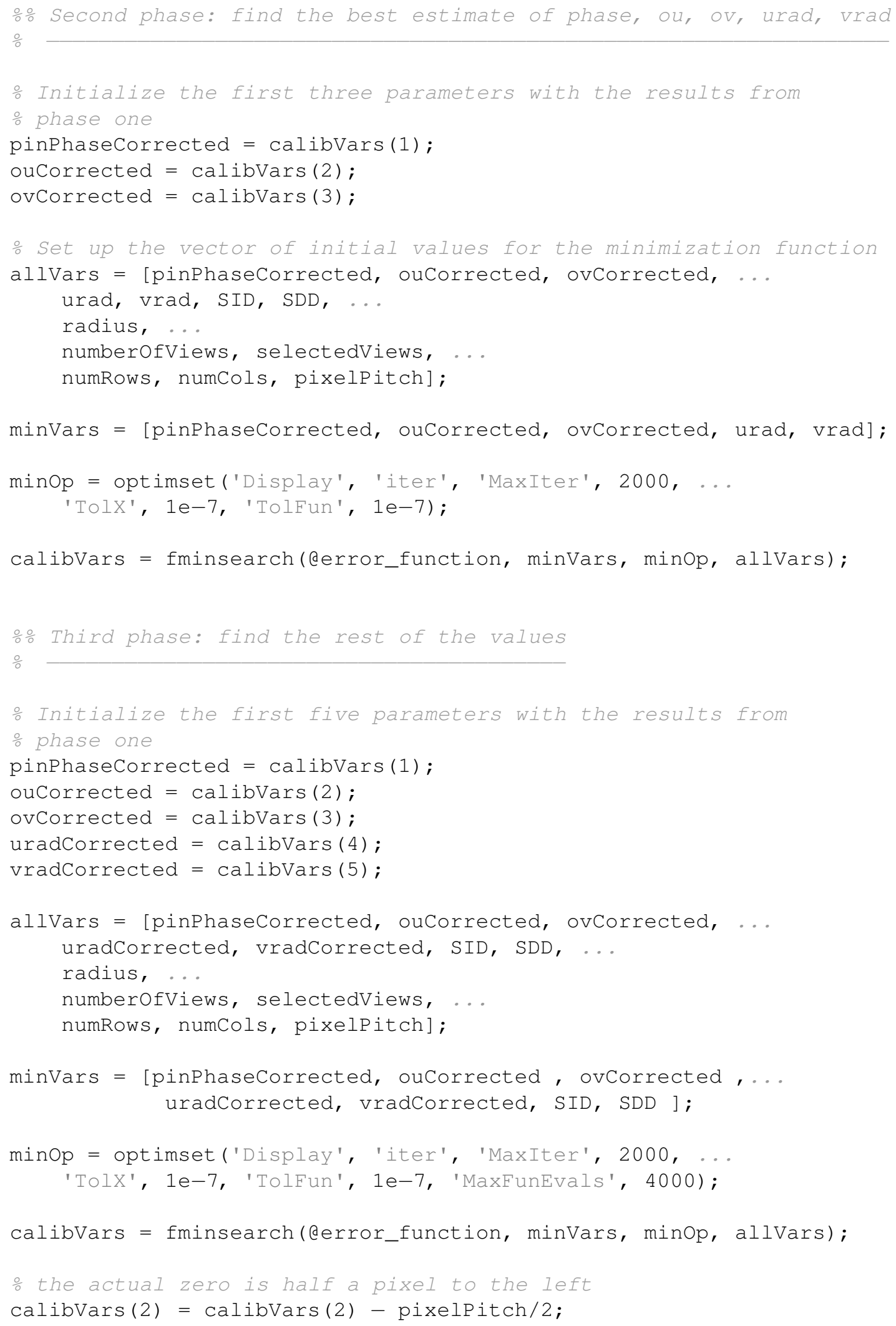




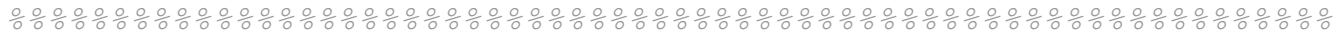

응 Internal functions

응응응응응응응응응응응응응응응응응응응응응응응응응응응응응응응응응응응응응응응응응응응응응응응응응응응응응응응응응응응응응응응응

function errorVal = error_function (minVars, allVars)

global p1 iterCount;

global pincoords ballHeights;

global flag_SHOW_PROGRESS

응 initial values

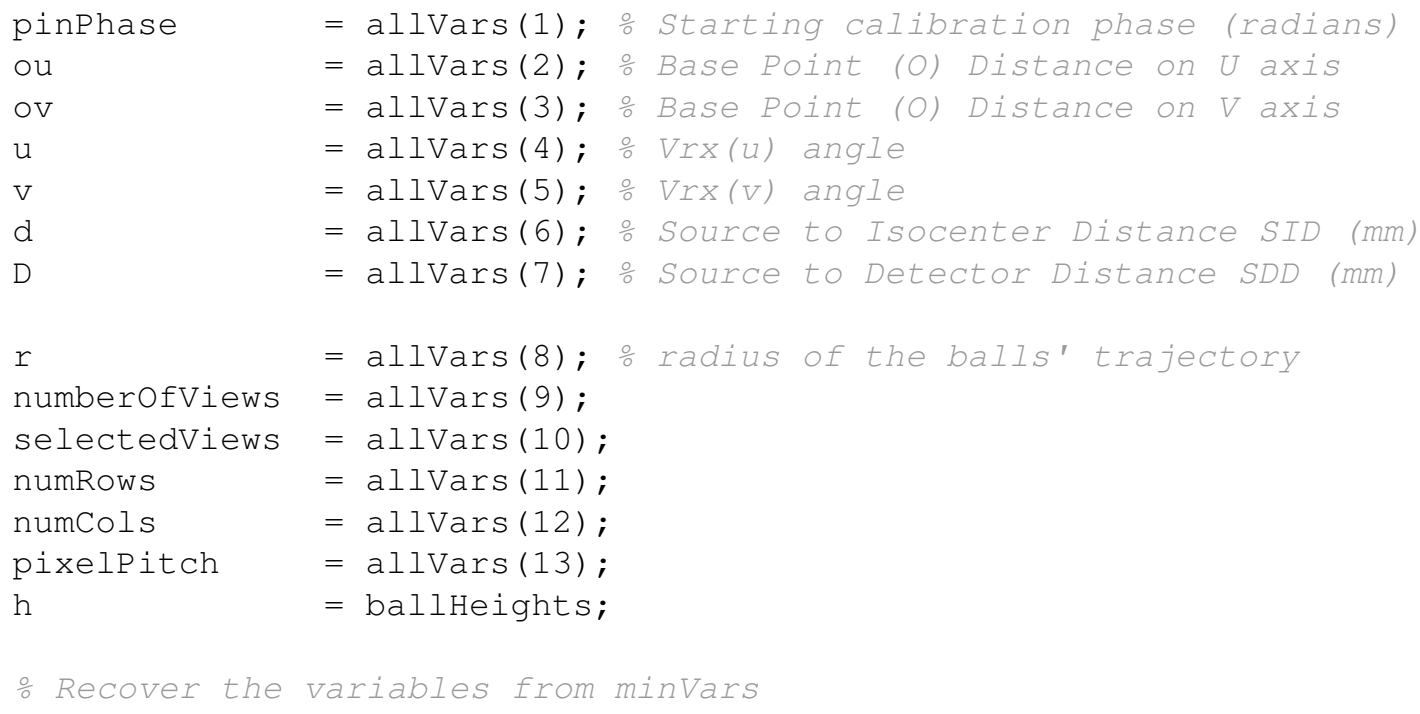




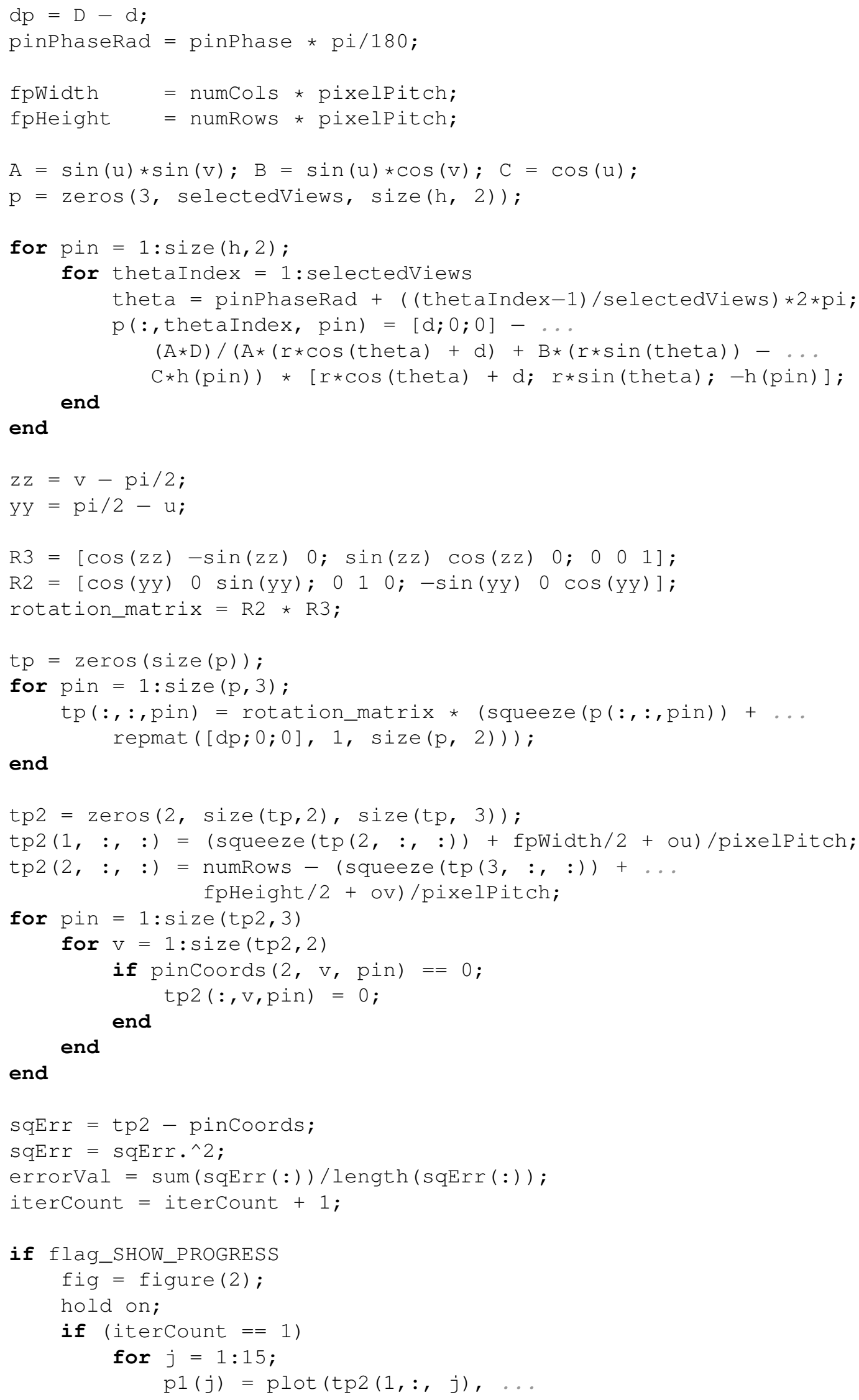




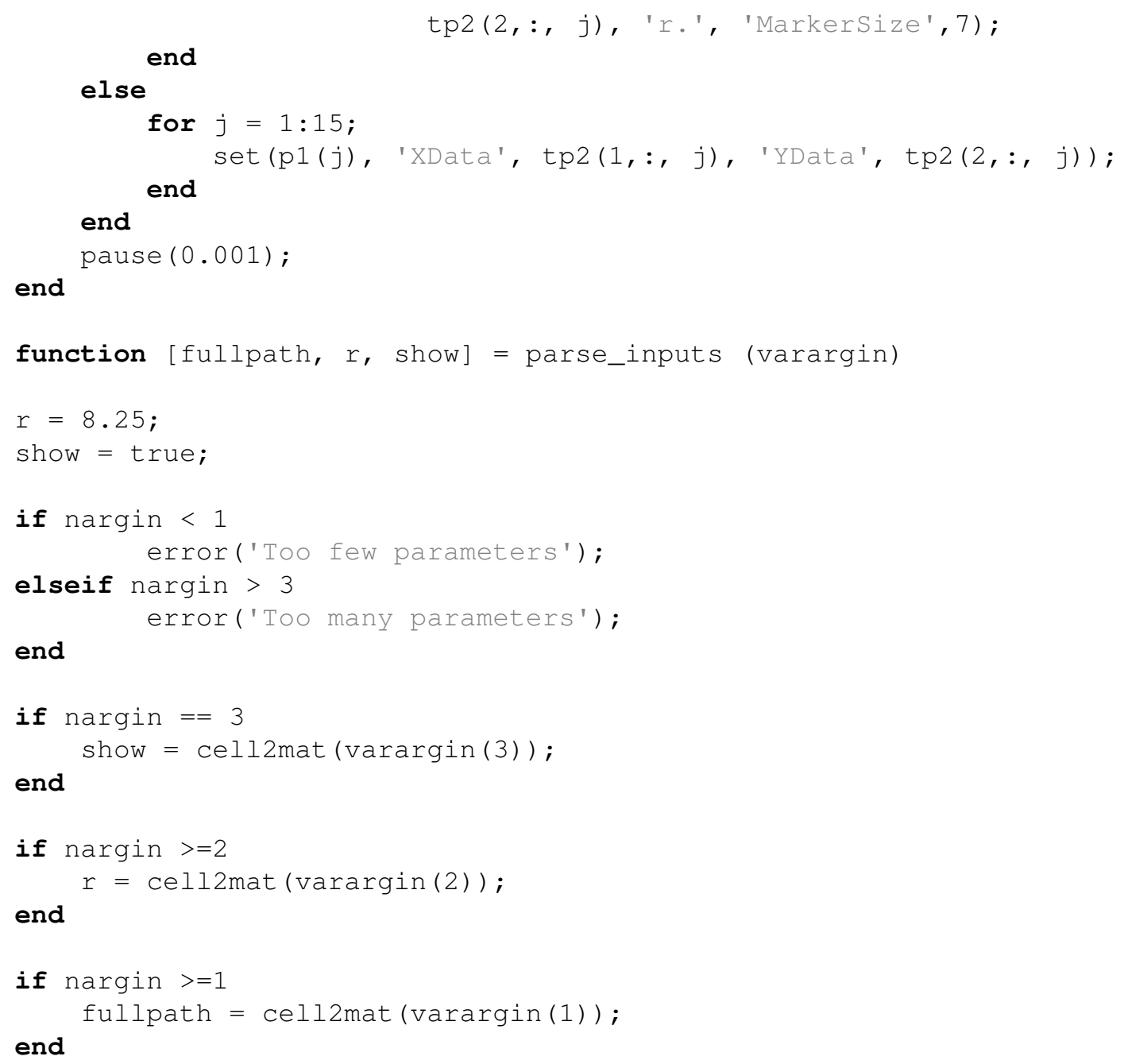




\section{Appendix B}

\section{VRX-CBCT Reconstruction Code}

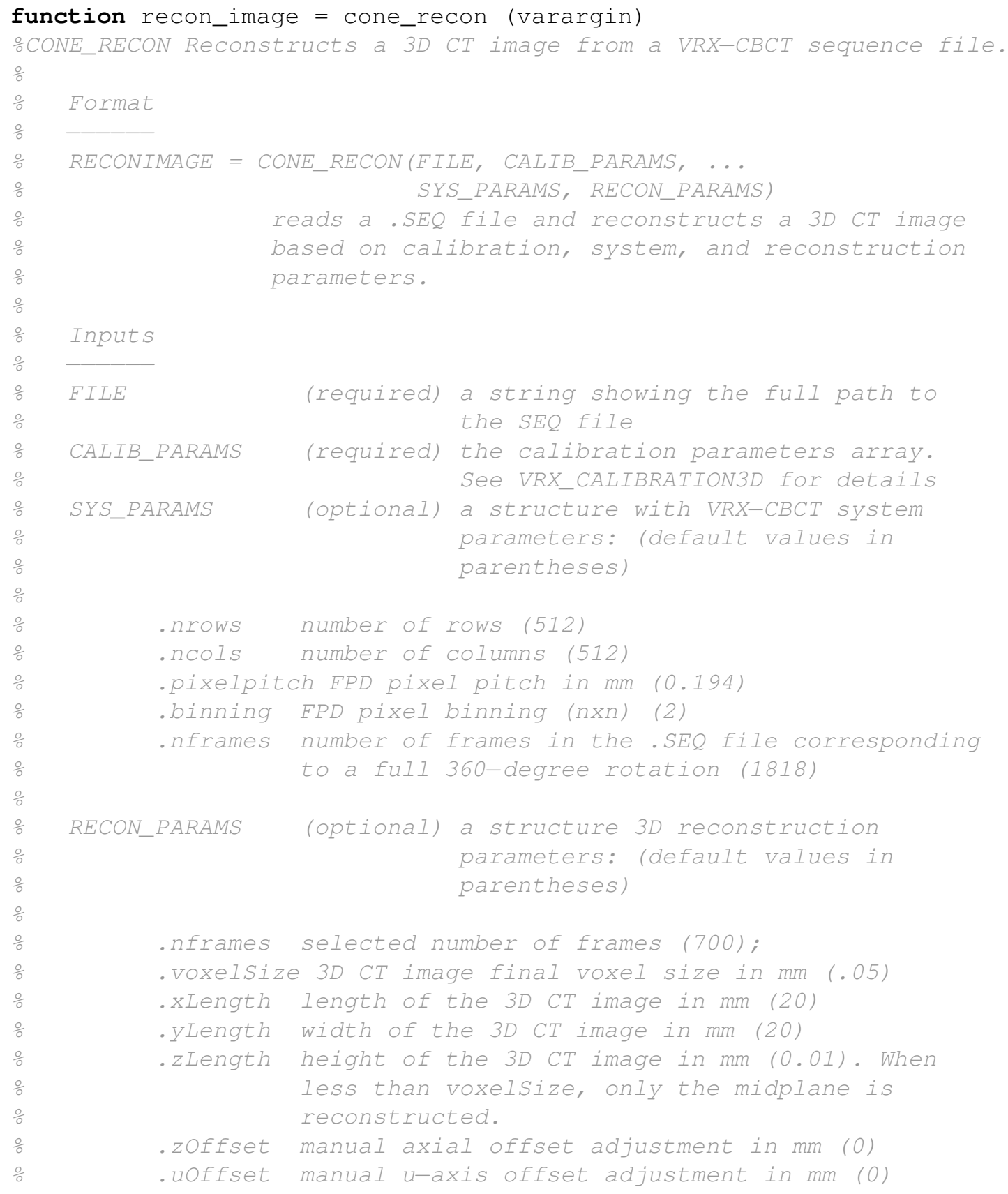




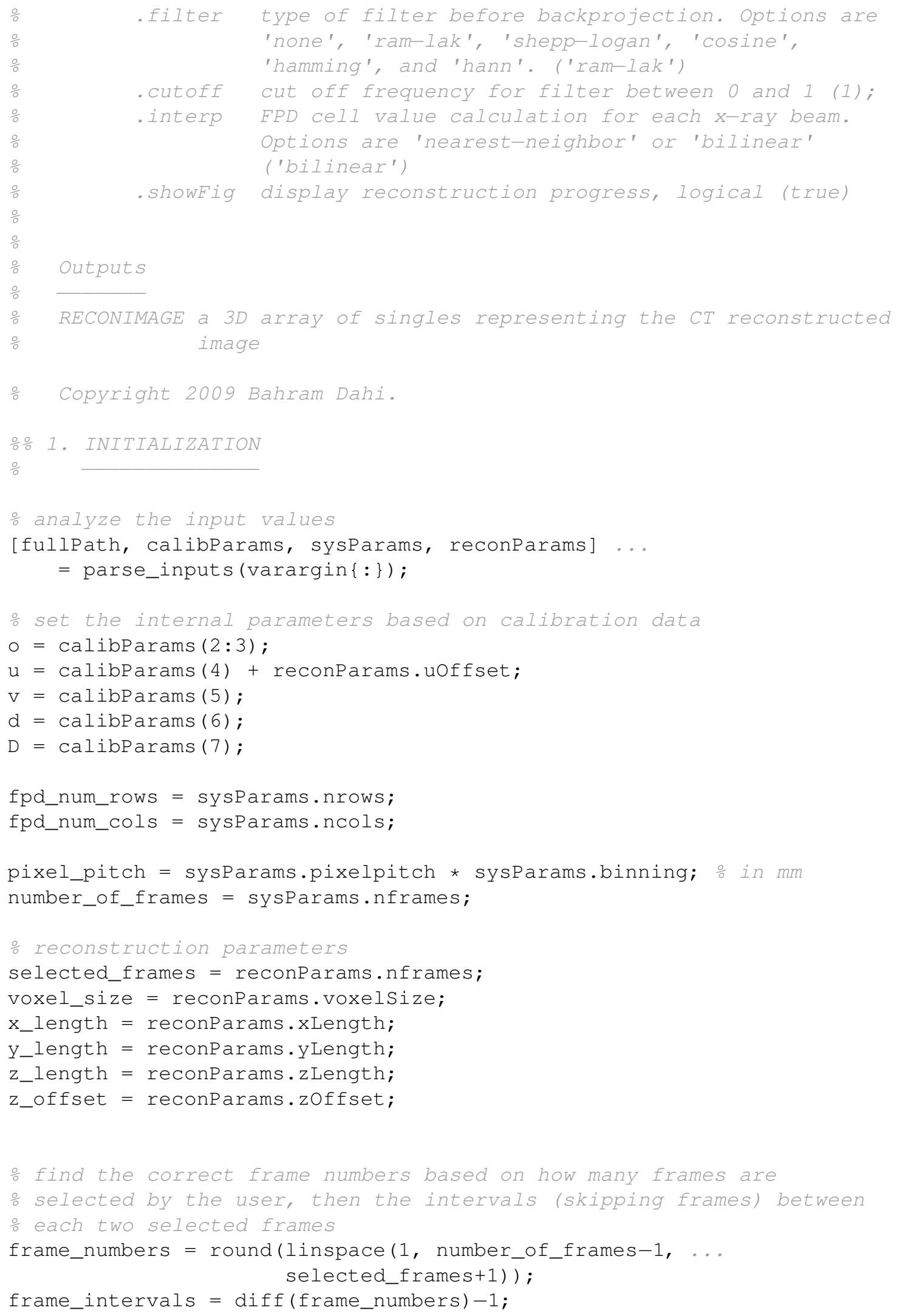




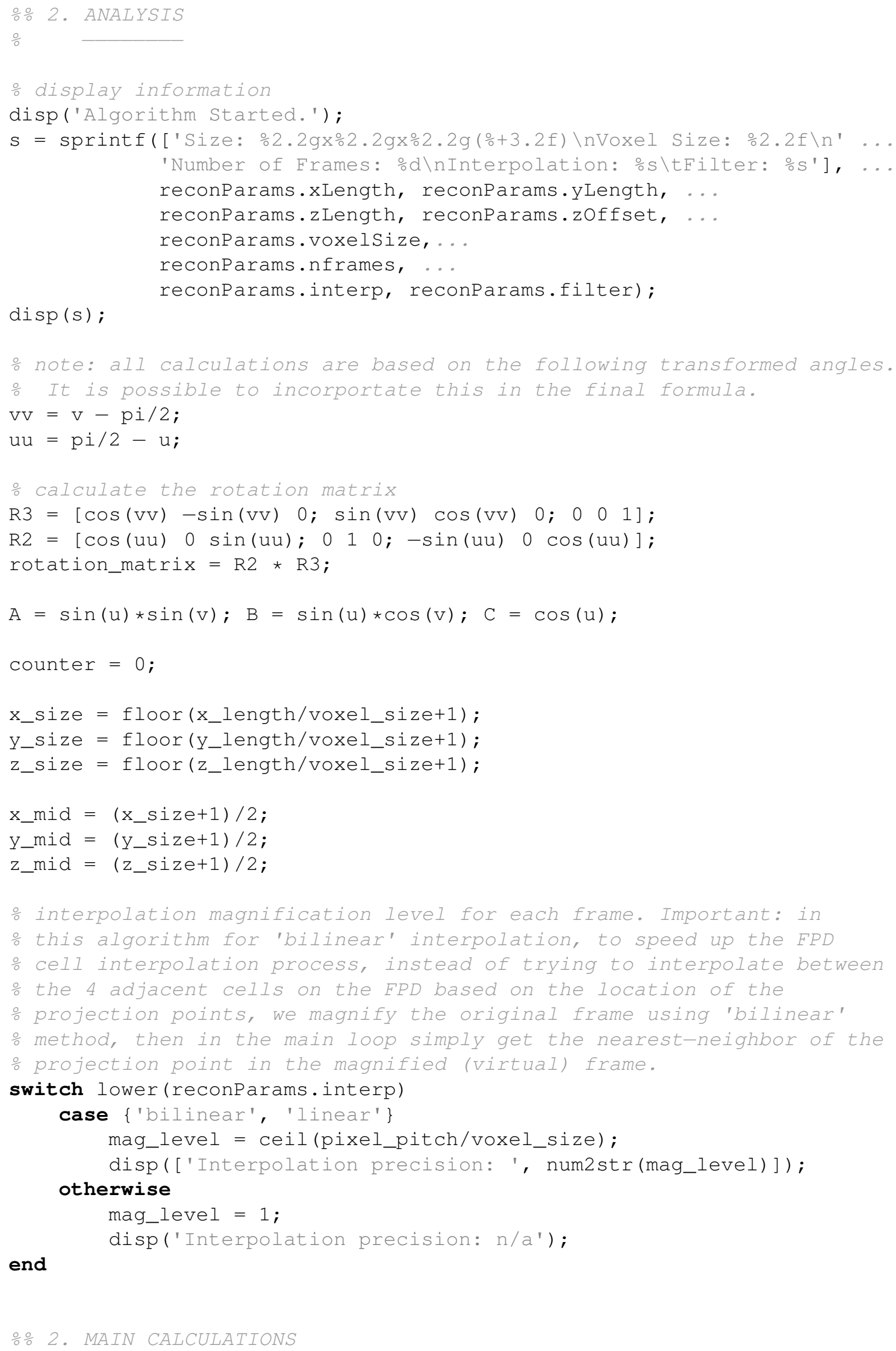




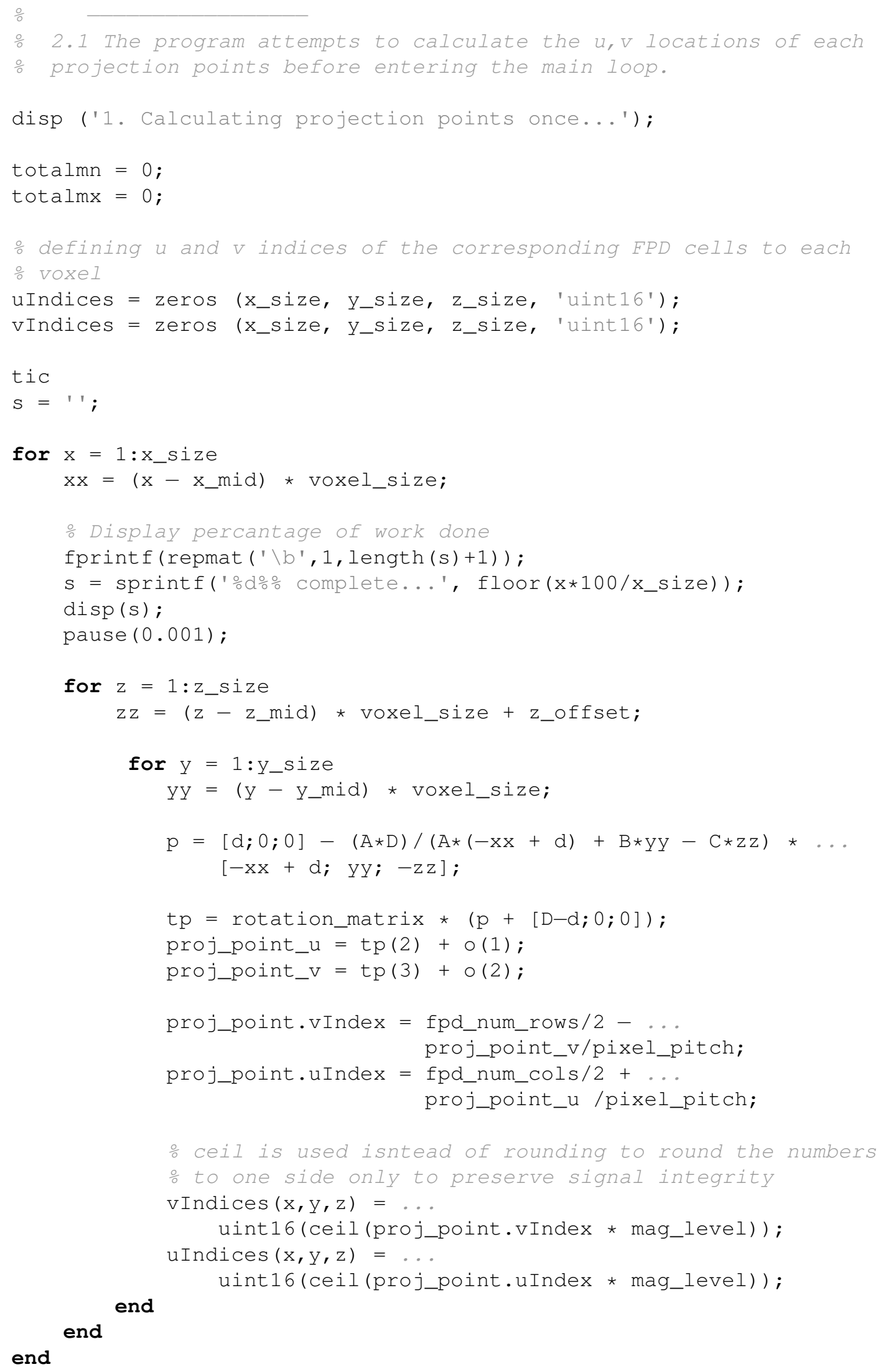




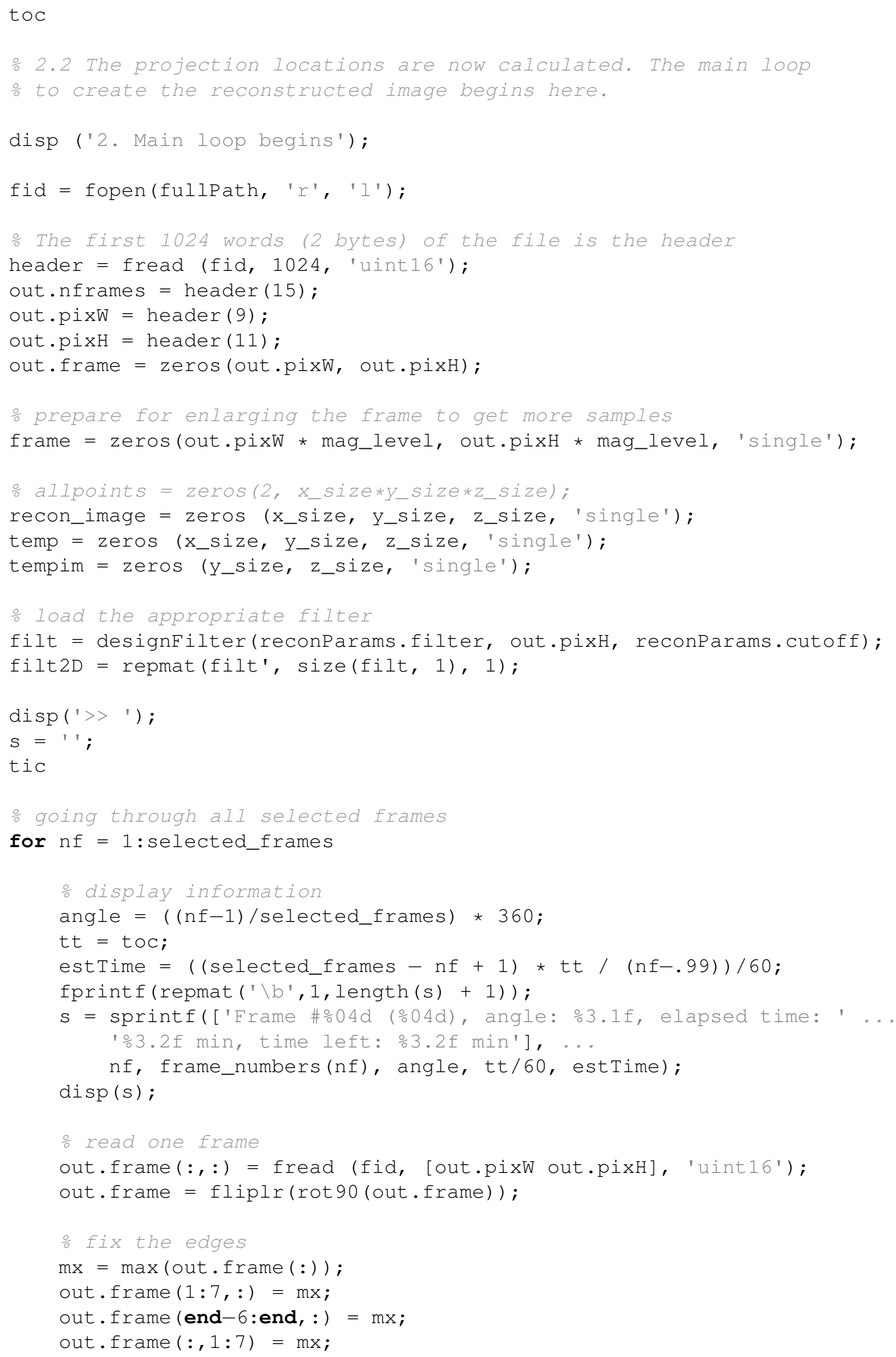




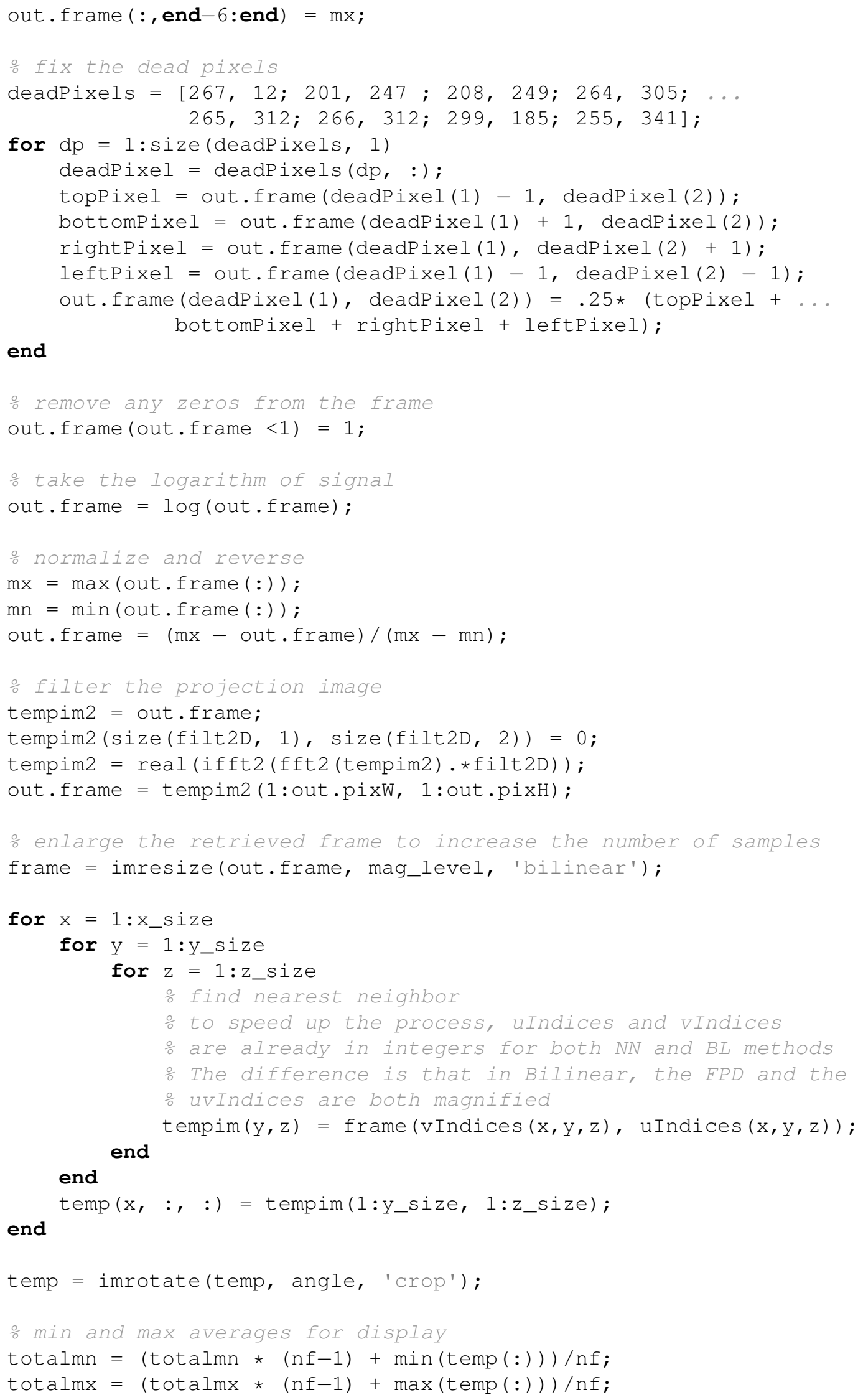




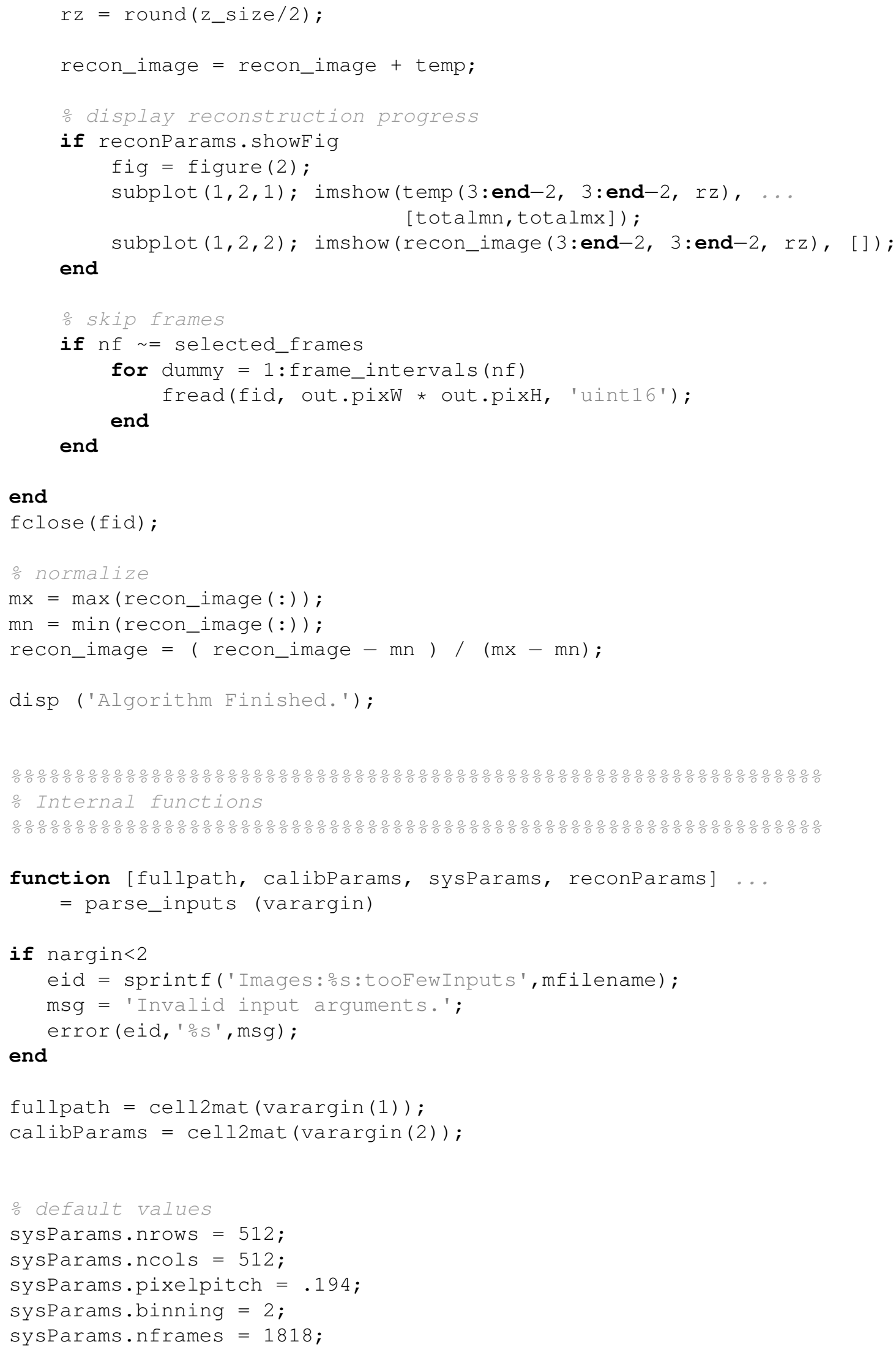




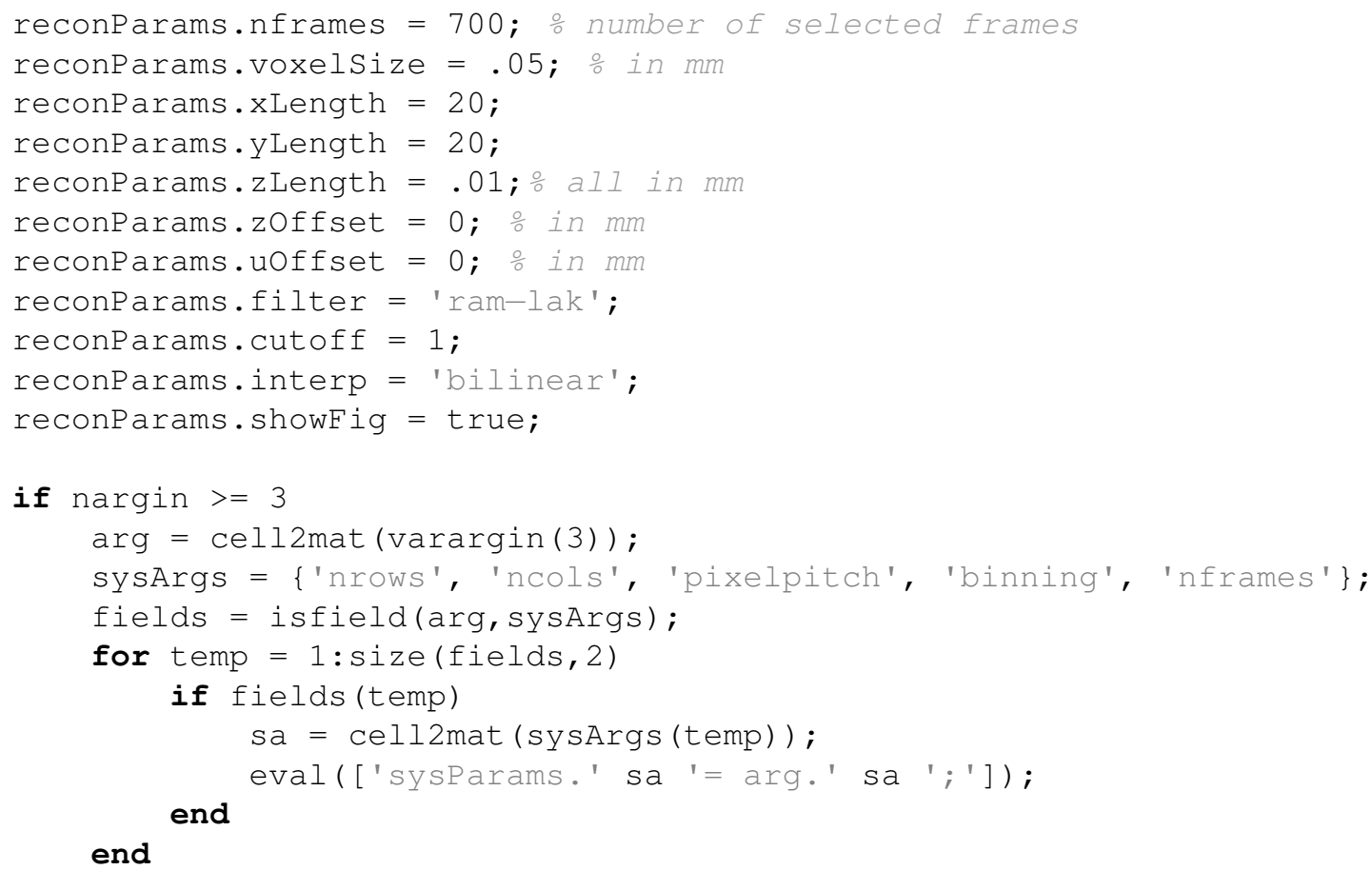




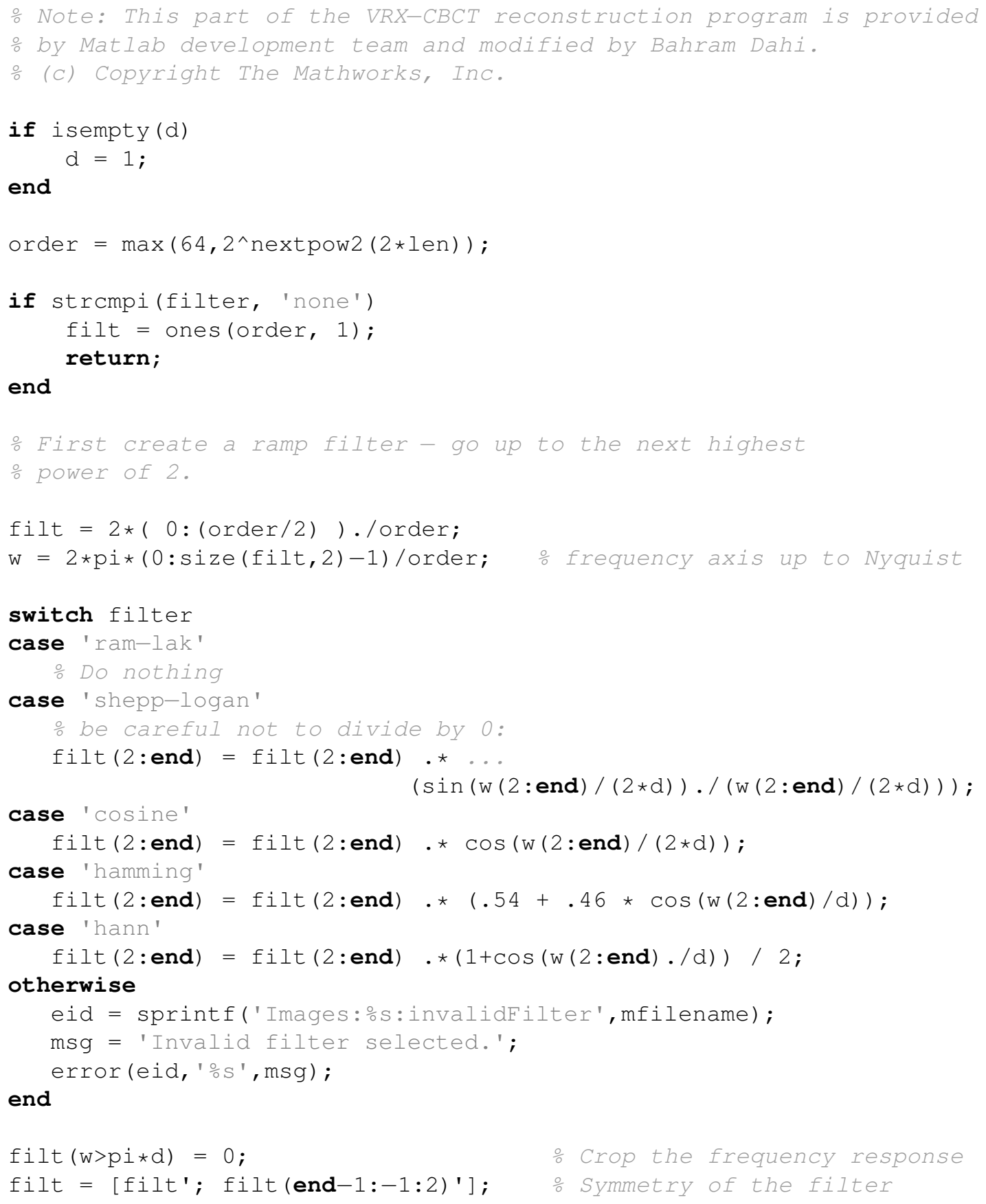




\section{Vita}

Bahram Dahi was born in 1977 in Tehran, Iran. He entered the Sharif University of Technology in 1995. After getting his Bachelor of Science degree in Civil Engineering in 2000, he decided to pursue his graduate studies in the field of Computer Science. He was admitted to the University of North Carolina at Charlotte in 2001, and received his Master of Science degree in Computer Science in 2003. Immediately after that, in January 2004, he moved to Memphis, TN to attend the University of Tennessee Health Science Center, where he continued his graduate studies at the Doctor of Philosophy level in Biomedical Engineering. His research was primarily in the BioImaging field, where he effectively shifted his focus from a theoretical approach to Computer Science research to a more practical use of his Computer Science knowledge combined with his Engineering background. 